\title{
A comparison of conflict diffusion models in the flanker task through pseudo-likelihood Bayes factors
}

\author{
Nathan J. Evans ${ }^{a}$ and Mathieu Servant ${ }^{b}$ \\ ${ }^{a}$ Department of Psychology, University of Amsterdam, The Netherlands \\ ${ }^{b}$ Department of Psychology, Université de Franche-Comté, France
}

Word count: 13,400

We would like to thank Corey White for providing the data from White et al. (2011) and Rolf Ulrich for providing the data from Ulrich et al. (2015).

Correspondence concerning this article may be addressed to: nathan.j.evans@uon.edu.au (NJE) or mathieu.servant@univ-fcomte.fr (MS) 


\begin{abstract}
Conflict tasks are one of the most widely studied paradigms within cognitive psychology, where participants are required to respond based on relevant sources of information while ignoring conflicting irrelevant sources of information. The flanker task, in particular, has been the focus of considerable modeling efforts, with only three models being able to provide a complete account of empirical choice response time distributions: the dualstage two-phase model (DSTP), the shrinking spotlight model (SSP), and the diffusion model for conflict tasks (DMC). Although these models are grounded in different theoretical frameworks, can provide diverging measures of cognitive control, and are quantitatively distinguishable, no previous study has compared all three of these models in their ability to account for empirical data. Here, we perform a comparison of the precise quantitative predictions of these models through Bayes factors, using probability density approximation to generate a pseudo-likelihood estimate of the unknown probability density function, and thermodynamic integration via differential evolution to approximate the analytically intractable Bayes factors. We find that for every participant across three data sets from three separate research groups, DMC provides an inferior account of the data to DSTP and SSP, which has important theoretical implications regarding cognitive processes engaged in the flanker task, and practical implications for applying the models to flanker data. More generally, we argue that our combination of probability density approximation with marginal likelihood approximation - which we term pseudo-likelihood Bayes factors provides a crucial step forward for the future of model comparison, where Bayes factors can be calculated between any models that can be simulated. We also discuss the limitations of simulation-based methods, such as the potential for approximation error, and suggest that researchers should use analytically or numerically computed likelihood functions when they are available and computationally tractable.
\end{abstract}


Keywords:

Conflict diffusion models — Marginal likelihood approximation — Probability density approximation — Flanker task 


\section{Introduction}

Understanding human decision-making has been a topic of enduring interest for several different fields of research, such as psychology, neuroscience, and economics (Tversky \& Kahneman, 1974; Ratcliff, 1978; Kahneman \& Tversky, 1979; Roitman \& Shadlen, 2002; Purcell et al., 2010; Hawkins, Forstmann, Wagenmakers, Ratcliff, \& Brown, 2015; Ratcliff, Smith, Brown, \& McKoon, 2016). One of the most widely studied decision paradigms within cognitive psychology involves both decision-relevant and decision-irrelevant sources of information, with the irrelevant sources of information either being consistent with, or in conflict to, the relevant sources of information. Several experimental paradigms have been developed to create different types of conflicting information, such as the Stroop task (Stroop, 1935), the Simon task (Simon \& Rudell, 1967; Simon, 1969), and the flanker task (B. A. Eriksen \& Eriksen, 1974), with the general finding that conflict slows down and impairs responding. Our focus in this article will be on the flanker task, where participants are instructed to respond according to the identity of a central target (e.g., a left response to the letter $\mathrm{H}$, and a right response to the letter $\mathrm{S}$ ) flanked on each side by distractors either associated with the same (compatible display) or the opposite response (incompatible display). The flanker task has been the focus of several key findings on conflict processing, and more importantly, the basis for the development of several computational models. Specifically, we aim to provide the first quantitative comparisons between all three diffusion models of the flanker task, and to perform this comparison using pseudo-likelihood Bayes factors, in the first (to the best of our knowledge) application of marginal likelihood approximation techniques to cognitive models without a tractable likelihood function.

Several computational models have been developed to better understand how conflicting information influences decisions in the flanker task (Yu, Dayan, \& Cohen, 2009; Hübner, Steinhauser, \& Lehle, 2010; White, Ratcliff, \& Starns, 2011; Ulrich, Schröter, 
Leuthold, \& Birngruber, 2015; Logan, 1980, 1996; Cohen, Servan-Schreiber, \& McClelland, 1992). However, to date only three models provide a complete account of the choice response time distributions (i.e., response time and accuracy data; see Hübner et al., 2010 and White, Brown, \& Ratcliff, 2012 for models of the flanker task that do not capture response time distributions): the dual-stage two-phase model (DSTP; Hübner et al., 2010), the shrinking spotlight model (SSP; White et al., 2011), and the diffusion model for conflict tasks (DMC; Ulrich et al., 2015). These models all build upon the diffusion framework (Stone, 1960; Laming, 1968; Ratcliff, 1978; Ratcliff \& Rouder, 1998; Ratcliff \& Tuerlinckx, 2002), which proposes that information is integrated for each alternative at some rate (known as the "drift rate") until the evidence for one alternative reaches some level (known as the "threshold"), and a decision is triggered. Importantly, the diffusion model has been shown to provide an accurate account of choice response time distributions in a range of non-conflict tasks (Ratcliff, 1978; Ratcliff \& Rouder, 1998; Evans \& Brown, 2017; Evans, Hawkins, Boehm, Wagenmakers, \& Brown, 2017). Each model extends upon the diffusion framework by assuming that the drift rate is the result of both task-relevant and task-irrelevant information, with the influence of task-irrelevant information being a time-varying process via mechanisms of either selective attention or automatic processing. However, these models differ in their theoretical basis for how the irrelevant stimulus information affects the decision variable, and therefore, have different mathematical functional forms that make different quantitative predictions about the shapes of the choice response time distributions.

DSTP (Hübner et al., 2010) is theoretically based on visual attention research, and proposes that information processing is performed in two phases, with the first phase having two separate sub-processes. In the first phase attention is spread, and the integration of information is based on information from both the target and the irrelevant stimuli. While 
information is being integrated to select the correct alternative, a second (also diffusion) identification process attempts to select a stimulus to narrow attention on. If this second process finishes before a decision is made, the second phase of information integration begins. In the second phase attention is focused on the stimulus previously identified as the target, which results in fast evidence accumulation either for (if the target was focused on) or against (if a distractor was focused on) the correct responses. The theoretical framework of DSTP is generalizable to all conflict tasks (Hübner et al., 2010; Hübner \& Töbel, 2019).

SSP (White et al., 2011) is also theoretically grounded in visual attention research, and proposes that the amount of relevant and irrelevant information integrated is the result of a gradually shrinking spotlight of attention (C. W. Eriksen \& James, 1986). At the beginning attention is spread, and the integration of information is based upon the amount of attention focused on the target compared to the flankers. As time continues the attentional spotlight continues to shrink and the integration of information is increasingly based on only the target. The theoretical framework of SSP has been specifically developed to account for data in the flanker task, making the flanker task ideal for providing a fair comparison of all three models.

DMC (Ulrich et al., 2015) is theoretically based on automaticity research, and proposes that independent information for task-relevant and task-irrelevant factors are summed during the decision process, which creates some overall time-varying accumulation process. Specifically, information is integrated for both a controlled process and an automatic process. The controlled process is only based on the target stimulus and remains constant over time, whereas the automatic process is based on irrelevant stimuli and varies over time (according to a scaled gamma function), with the largest impact being in early stages and almost no impact remaining in later stages. The theoretical framework of 
DMC is generalizable to all conflict tasks (Ulrich et al., 2015; Servant, White, Montagnini, \& Burle, 2016; Ambrosi, Servant, Blaye, \& Burle, 2019; Servant, van Wouwe, Wylie, \& Logan, 2018), and was specifically developed to be a general account of all conflict tasks.

Previous studies involving these models have both attempted to assess their ability to explain trends in empirical data, as well as the relationship between them and their measurement of cognitive control (i.e., ability to suppress conflict-based interference). White, Servant, and Logan (2018) attempted to provide a detailed comparison in the latter category, assessing the mimicry between the models in fit and inferences. Specifically, White et al. (2018) found that the models could be quantitatively distinguished from one another, with a cross-fitting procedure showing that each model was able to better fit its own data than the other models, suggesting that each model makes unique quantitative predictions about the choice response time distributions. Interestingly, White et al. (2018) found that these differences were also present between some models in the measurement of cognitive control: although the measurements from DSTP and SSP were highly correlated, the measurements from DMC greatly diverged from both DSTP and SSP. Therefore, it is important to determine whether certain models provide a more accurate explanation than others, as there are meaningful practical differences between the models in the conclusions drawn about cognitive control.

As mentioned above, the findings of White et al. (2018) showed that DSTP, SSP, and DMC make quantitatively distinguishable predictions about the response time distributions. Several previous studies have attempted to compare these precise quantitative predictions of DSTP and SSP in their ability to account for empirical response time distributions. Findings have been mixed, with some studies providing a slight superiority of DSTP (Hübner \& Töbel, 2012; Servant, Montagnini, \& Burle, 2014; Servant, White, Montagnini, \& Burle, 2015), and others providing a superiority of SSP (White et al., 2011). 
However, no previous studies have compared DMC to DSTP and/or SSP, which is the most theoretically and practically important comparison, as White et al. (2018) found DMC to produce different inferences about cognitive control than DSTP and SSP.

In addition, previous comparisons of DSTP and SSP have contained limitations regarding their implementations of the models. Firstly, previous studies have only used the "simple diffusion" variants of the models (though see White et al., 2011, Exp5, for a "full diffusion" SSP), which does not allow between-trial variability in any of the parameter values. However, previous research involving the diffusion model has found that betweentrial variability in the parameters for the rate of evidence accumulation (i.e., drift rate; Ratcliff, 1978), the starting amount of evidence (i.e., starting point; Ratcliff \& Rouder, 1998), and the time dedicated to non-decision processes (i.e., non-decision time; Ratcliff \& Tuerlinckx, 2002) are required to meet key benchmark phenomena. Secondly, the models have been fit via quantile-based methods (i.e., $\chi^{2} / G^{2}$ ), which when used to make inferences about the entire response time distribution make the implicit assumption that the distribution can be described in a minimal number of response time quantiles: that is, that the summary statistic based on these quantiles is a sufficient statistic for the response time distributions. Although these quantile-based statistics may lead to identical results in most situations, this is not guaranteed for all cases (unless the statistic is sufficient), and may be questionable for conflict models where the key distinction is in the precise shape of the distribution (see Robert, Cornuet, Marin, \& Pillai, 2011 for potential issues that arise when summary statistics are not sufficient). Lastly, quantitative comparisons between the models have been made with "parameter counting" methods of model selection, such as the Bayesian Information Criterion (BIC; G. Schwarz, 1978), which assume that the flexibility of the model is purely a function of its number of free parameters. However, these methods ignore the concept of "functional form flexibility", where the true flexibility of 
the model is a combination of both its free parameters and the "functional form" of the model, with "functional form" referring to the mathematical equations of the model and how the parameters combine within them. In essence, different functional forms will result in parameters that are able to have a larger/smaller impact on the predictions of the model, which is ignored by methods that treat all free parameters equally (Myung \& Pitt, 1997; Myung, 2000; Evans, Howard, Heathcote, \& Brown, 2017).

Our study aims to provide the first comprehensive quantitative comparison between the three key models of the flanker task: DSTP, SSP, and DMC. Importantly, previous research has found differences between the models based on visual attention (DSTP and SSP) and the model based on automaticity (DMC) in their measurement of cognitive control, meaning that it is crucial to understand which model(s) provide a better account of the data from the flanker task. Our study uses flanker data from three previous studies - each from a different group of researchers (White et al., 2011; Servant et al., 2015; Ulrich et al., 2015) - to ensure generalizablility of inferences, and also makes large improvements on the previous studies that compared DSTP and SSP. Firstly, we implement each of the models both in the "simple" (i.e., no between-trial variability parameters) and "full" (i.e., between-trial variability in drift rate, starting point, and non-decision time) diffusion frameworks, as opposed to previous studies that only used the simple versions.

Secondly, and most notably, we compare these models through a unique combination of probability density approximation and marginal likelihood estimation, which we term pseudo-likelihood Bayes factors. Specifically, we apply recently developed probability density approximation (PDA; Turner \& Sederberg, 2014; Holmes, 2015) methods to obtain a pseudo-likelihood for each of the models through simulation. PDA allows these models - which have intractable likelihood functions - to be fit with likelihood-based methods that use the entire choice response time distributions, rather than the quantile-based 
methods used in previous studies. Importantly, we combine the pseudo-likelihood obtained through PDA with a recently developed method for efficiently estimating marginal likelihoods, TIDE (Evans \& Annis, 2019), which allows us to compare these models with Bayes factors (Kass \& Raftery, 1995) instead of the "parameter counting" methods of previous studies. The "marginal likelihood" quantifies how likely the data are when assuming a specific model, which is integrated over all possible parameter values, meaning that it does not depend on a specific set of parameters. The marginal likelihoods of two models can then be expressed as a ratio, which provides the Bayes factor: a widely used method of model inference that is considered to provide an ideal balance between the ability to account for empirical data and the functional flexibility of the model (Kass \& Raftery, 1995; Myung \& Pitt, 1997; Gronau et al., 2017; Evans \& Brown, 2018). To the best of our knowledge, this is the first instance of Bayes factors being calculated with pseudo-likelihood methods for cognitive models, with previous applications of Bayes factors for response time models being limited to models with analytically solvable likelihoods (Evans \& Brown, 2018; Annis, Evans, Miller, \& Palmeri, 2019; Evans, Bennett, \& Brown, 2018; Evans \& Annis, 2019). Importantly, combining these methods provides the potential for broad improvements in the ability to compare computational models, where the marginal likelihood can be computed for any model that can be simulated.

\section{Method}

Our study provides a comparison between DSTP, SSP, and DMC using the flanker data from three previous studies (and three different research groups): White et al. (2011), Servant et al. (2015), and Ulrich et al. (2015). Here, we first briefly detail each of these studies. Following this, we outline the mathematical functions of each of the models that our study compares. Next, we provide a detailed description of the two key methods that 
we combine to compute pseudo-likelihood Bayes factors - PDA and TIDE - including a discussion about the general classes of methods that each of these specific methods originate from: simulation-based Bayesian methods and marginal likelihood estimation methods, respectively. Lastly, we detail how the pseudo-likelihood Bayes factor model comparison was performed within our study.

For all experiments we excluded trials that were faster than $150 \mathrm{~ms}$ or slower than $1500 \mathrm{~ms}$, in order to create some level of consistency between studies. Participant exclusion criteria were identical to those used in the original studies.

\section{Details of studies}

White et al. (2011). We analyse the data from Experiment 1 of White et al. (2011), where 25 participants completed a standard flanker experiment. Specifically, participants were presented with a sequence of five arrows (i.e., $<$ or $>$ ) presented vertically. Participants were instructed to identify whether the central arrow, which was always presented in the centre of the screen, was pointing towards the left or right of the screen. Participants were told to do so as quickly and accurately as possible, though there was no explicit deadline for responses, and the stimulus remained on screen until a response was made. After each response there was a $350 \mathrm{~ms}$ inter-trial interval, with the next stimulus being presented immediately after this interval (i.e., no central fixation cue before the next stimulus). Compatible trials were those where the target and flankers pointed in the same direction, whereas incompatible trials were those where the flankers pointed in the opposite direction to the target. Participants completed 48 practice trials followed by 8 blocks of 96 trials each.

Ulrich et al. (2015). We analyse the flanker data from the only experiment of Ulrich et al. (2015), where 18 participants completed a flanker task consisting of the letters ' $\mathrm{H}$ ' 
and 'S', though two participants were excluded for high error rates (>10\%). Participants were presented with a string of 5 letters presented horizontally, and had to identify whether the central letter, which was always presented in the centre of the screen, was a ' $\mathrm{H}$ ' or an 'S'. Each trial began with a fixation cross presented in the center of the screen for $500 \mathrm{~ms}$, followed by the presentation of the stimulus array for $150 \mathrm{~ms}$. A response deadline was set at $1,500 \mathrm{~ms}$, where participants were required to respond within $1,500 \mathrm{~ms}$ of the stimulus array presentation or the trial would be counted as a "miss", and the next trial would begin. After each trial there was a 1,000ms inter-trial interval before the onset of the fixation cross for the next trial. Participants were provided with visual feedback during this time period if their previous response was anticipatory $(<50 \mathrm{~ms})$, a miss, or incorrect. Compatible trials were those where the target and flankers matched, whereas incompatible trials were those where the flankers supported the opposite response to the target (e.g., an 'S' target flanked by 'H'). Participants completed 56 practice trials followed by 4 blocks of 56 flanker trials each. Participants in this experiment also completed a Simon task, which was presented in alternating blocks with the flanker task, though we do not analyse the Simon data within this study.

Servant et al. (2015). We analyse the data from the only experiment of Servant et al. (2015), where 12 participants completed a flanker task consisting of the letters ' $\mathrm{H}$ ' and 'S'. Participants were presented with a string of 5 letters presented horizontally, and had to identify whether the central letter, which was always presented in the centre of the screen, was a 'H' or and 'S'. Participants were told to do so as quickly and accurately as possible, though there was no explicit deadline for responses, and the stimulus remained on screen until a response was made. After each response there was a 1,000ms inter-trial interval, with the next stimulus being presented immediately after this interval (i.e., no central fixation cue before the next stimulus). Compatible trials were those where the target and 
flankers matched, whereas incompatible trials were those where the flankers supported the opposite response to the target (e.g., an 'S' target flanked by 'H'). Participants completed 96 practice trials followed by 15 blocks of 96 trials each. Participants were also subject to electromyographic (EMG) recordings to assess their motor movement in responding, though we do not analyse these data within this study.

\section{Model definitions}

Each of the three models - DSTP, SSP, DMC - were defined within the diffusion framework. The diffusion model (Ratcliff, 1978; Ratcliff et al., 2016) is one of the most widely applied models of decision-making, which is able to an analyse choice and response time data in unison, allowing it to account for the well-known speed-accuracy tradeoff. The diffusion model proposes that decisions are made through a process of evidence accumulation, where noisy evidence from the environment accumulates in favour of the two alternatives at some rate (known as the "drift rate"), until the evidence for one alternative reaches a threshold level of evidence, and a decision is triggered for that alternative. Formally, the evidence accumulation process can be written as a stochastic differential equation:

$$
d x=v d t+c d W
$$

where $x$ is the evidence, $W$ is the Wiener process (noise from the standard normal distribution that evolves with the square root of time), and $c$ is the diffusion coefficient, with $c$ being fixed to 0.1 to solve a scaling property within the model.

The "simple" diffusion model contains 4 free parameters, which correspond to the drift rate $(v)$, the threshold level of evidence $(a)$, the starting amount of evidence at the beginning of the process $(z)$, and the time required for processes unrelated to the decision 
process, such as perceptual encoding and motor activation (ter). The "full" diffusion model includes 3 additional parameter, which provide between-trial variability in the drift rate $\left(s_{v}\right)$, starting point $\left(s_{z}\right)$, and non-decision time $\left(s_{t e r}\right)$ parameters. The between-trial variability in drift rate is defined as a normal distribution with mean $v$ and standard deviation $s_{v}$, whereas the between-trial variability in both starting point and non-decision time are defined as uniform distributions, centered on $z$ and ter with widths $s_{z}$ and $s_{t e r}$, respectively.

Note that the piecewise (DSTP) or time-varying (SSP and DMC) drift rate contained within each of the conflict diffusion models make their likelihood functions computationally intractable, and hence our use of PDA to create simulation-based pseudo-likelihood functions. Specifically, the likelihood function derived for DSTP in Hübner et al. (2010) requires computing the density for three different diffusion processes, with the final diffusion process containing computationally burdensome integrals, as they depend on the states of the previous two diffusion processes. This becomes even more computationally burdensome when combined with the three dimensional integral required for the "full diffusion" variant, which must be computed numerically, meaning that although it is technically possible to calculate the likelihood for DSTP, the large amount of numerical integration required makes the process computationally intractable. For models with time-varying drift rates, such as SSP and DMC, previous research has suggested that the likelihood function is mathematically intractable (Ratcliff, 1980; Ulrich et al., 2015). Although numerical solutions can technically be computed for SSP and DMC using the equations of Smith (2000), this method for computing the likelihood function comes with a high computational burden, which becomes computationally intractable in the case of the "full diffusion" variants (Evans \& Hawkins, 2019; Evans, Hawkins, \& Brown, 2019) 
DSTP. DSTP (Hübner et al., 2010) proposes that information processing is performed in two phases, with the first phase having two separate sub-processes. In the first phase the integration of information is based on both the target and the flankers, and in the second phase it is based on only a single, selected stimuli. Formally, this can be written as a piecewise drift rate with two stages:

$$
v_{p 1}=v_{t a}+v_{f l} ; v_{p 2}=v_{p 2}
$$

where $v_{p 1}$ is the overall drift rate in the first phase, $v_{t a}$ is the rate of evidence accumulation for the target, $v_{f l}$ is the rate of evidence accumulation for the flankers, and $v_{p 2}$ is the drift rate for the second phase (discussed below). The flanker drift rate $\left(v_{f l}\right)$ is positive for compatible trials, and negative for incompatible trials.

While information is being integrated to select the correct alternative, a second diffusion process attempts to identify a stimulus to narrow attention on, and if this second process reaches the threshold before a decision is made, the second phase of information integration begins. Formally, this second process has a drift rate $v_{s s}$, where reaching the upper threshold results in the selection of the target to focus on, and reaching the lower threshold results in the selection of a flanker.

In the second phase, a single drift rate $\left(v_{p 2}\right)$ takes over from the overall drift rate from the first phase (i.e., $\left.v_{p 1}\right)$. The second phase drift rate $\left(v_{p 2}\right)$ is always positive for compatible trials, as both the targets and flankers have the same identity, meaning only the correct type of stimulus can be selected. For incompatible trials, the drift rate is positive when the upper threshold is reached by the stimulus selection process, and negative when the lower threshold is reached by the stimulus selection process.

Overall, this means that DSTP can have up to 9 free parameters: $v_{t a}, v_{f l}, v_{s s}, v_{p 2}, a$, $a_{s s}, z, z_{s s}, t e r$. However, we fixed $z_{s s}$ to be the mid-point of the two thresholds, assuming 
that participants could not be biased for the stimulus identification process, reducing the model to only 8 free parameters in the "simple" framework. To extend DSTP to the "full" diffusion framework, we added 3 between trial variability parameters, which were distributed in the same manner as the regular "full" diffusion: one for all drift rates $\left(s_{v}\right)$, one for all starting points $\left(s_{z}\right)$, and one for non-decision time $\left(s_{t e r}\right)$.

SSP. SSP (White et al., 2011) proposes that information processing is based on the amount of attention focused on the target compared to the flankers, with attention for the flankers decreasing over time as a "shrinking spotlight". Formally, this can be written as a time-varying drift rate, where the drift rate at time $t$ (i.e., $v(t))$ is given by:

$$
v(t)=s_{t a}(t) \times p_{t a}+s_{f l}(t) \times p_{f l}
$$

where $s_{t a}(t)$ is the area of the spotlight focused on the target at time $t, p_{t a}$ is the perceptual strength of the target, and $s_{f l}(t)$ and $p_{f l}$ are the same variables, respectively, for the flankers instead of the target. The $p_{t a}$ and $p_{f l}$ values are free parameters of the model, which are usually estimated as the same value, $p$, assuming that the target and flankers have equal perceptual strength. The flanker perceptual strength $\left(p_{f l}\right)$ is positive for compatible trials, and negative for incompatible trials.

The area of focus is governed by a normal distribution centred on 0 with a standard deviation that decreases over time, to reflect the shrinking spotlight of attention. Formally, the standard deviation can be given by:

$$
S D(t)=S D_{0}-S D_{r} \times t
$$

where $S D_{0}$ is the initial spotlight width, and $S D_{r}$ is the linear rate of decrease in the spotlight width, which are both free parameters of the model. When $S D(t)$ decreases to 
a value of 0.001 the spotlight of attention is assumed to have reached maximum focus, and $S D(t)$ stops decreasing for the remainder of the decision. From here, the area under the spotlight for the target and the flankers is calculated from integrating the normal distribution:

$$
\begin{array}{r}
s_{t a}(t)=\int_{-0.5}^{0.5} \Phi(x \mid 0, S D(t)) d x \\
s_{f l}(t)=\int_{-\infty}^{-0.5} \Phi(x \mid 0, S D(t)) d x+\int_{0.5}^{\infty} \Phi(x \mid 0, S D(t)) d x
\end{array}
$$

where $\Phi(x \mid \mu, \sigma)$ is the probability density function of the normal distribution. Each stimulus is assumed to be one unit wide, with the spotlight centrally focused on the target. In order to ensure that the total area of attention sums to 1, attention exceeding stimulus boundaries is allocated to the outer flankers, as in the original implementation of White et al., 2011. This gave the "simple" SSP 6 free parameters: $p, S D_{0}, S D_{r}, a, z$, and ter. To extend SSP to the "full" diffusion framework, we added 3 between trial variability parameters, which were distributed in the same manner as the regular "full" diffusion and were not time-varying: one for the combined drift rate $\left(s_{v}\right)$, one for the starting point $\left(s_{z}\right)$, and one for non-decision time $\left(s_{t e r}\right)$.

$D M C$. DMC (Ulrich et al., 2015) proposes that information is integrated for both a constant, controlled process based on the target stimulus, and a time-varying automatic process based on the flankers. Formally, this can be written as a time-varying drift rate, where the drift rate at time $t($ i.e., $v(t))$ is given by:

$$
v(t)=v_{c}+v_{a}(t)
$$


where $v_{c}$ is the drift rate for the controlled process, which is a free parameter of the model, and $v_{a}(t)$ is the drift rate for the automatic process at time $t$. The automatic process drift rate varies over time according to the first derivative of a (rescaled) gamma function:

$$
v_{a}(t)=\zeta \times e^{\frac{-t}{\tau}} \times\left[\frac{t \times e}{(\alpha-1) \times \tau}\right]^{(\alpha-1)} \times\left(\frac{\alpha-1}{t}-\frac{1}{\tau}\right)
$$

where $\alpha$ is the shape, $\zeta$ is the peak amplitude, and $\tau$ is the characteristic time. This gave the "simple" DMC 7 free parameters: $v_{c}, \alpha, \tau, \zeta, a, z$, and ter. To extend DMC to the "full" diffusion framework, we added 3 between trial variability parameters: one for all of the drift rates $\left(s_{v}\right)$, one for the starting point $\left(s_{z}\right)$, and one for non-decision time $\left(s_{t e r}\right)$. Note that these definitions of the between-trial variability parameters differ from the original definitions of Ulrich et al. (2015). To ensure that this difference did not influence our results, we also performed the analyses using definitions similar to Ulrich et al. (2015), and our key conclusions remained unchanged (see the Supplementary Materials).

\section{PDA and simulation-based Bayesian methods}

Probability density approximation (PDA; Turner \& Sederberg, 2014; Holmes, 2015) is a method for likelihood-free inference and estimation. Most common methods of inference and estimation - such as Bayes factors and Bayesian parameter estimation - are likelihood-based, meaning that in order to be applied they require the model to have a known and tractable likelihood function. Likelihood-free methods provide a means to apply likelihood-based methods to models with unknown or intractable likelihood functions, where a simulation-based process is used instead of the likelihood function. This means that the only requirement for likelihood-free methods is that the model can be simulated from. Most likelihood-free methods fall into one of two categories: Approximate Bayesian Computation (ABC) methods, which remove the need for a likelihood, or PDA methods, 
which replace the likelihood with a pseudo-likelihood.

ABC methods provide a likelihood-free methodology through removing the need for a likelihood, and instead use the discrepancy between the observed data and simulated data (Grelaud et al., 2009; Cornuet et al., 2008; Tavare, Balding, Griffiths, \& Donnelly, 1997; Beaumont, Zhang, \& Balding, 2002; Csilléry, Blum, Gaggiotti, \& François, 2010; Pritchard, Seielstad, Perez-Lezaun, \& Feldman, 1999; see Turner \& Sederberg, 2012; Turner \& Van Zandt, 2012; Turner, Dennis, \& Van Zandt, 2013; Turner \& Van Zandt, 2014 for examples in cognitive modelling). This typically involves reducing the data - both observed and simulated - to a single or set of summary statistic(s), and assessing the discrepancy between the observed and simulated data in these summary statistics. This discrepancy is then used to decide whether the proposed posterior sample will be accepted or rejected: if the parameter values produce a discrepancy that is below some predefined minimum level, then the posterior sample is accepted, and otherwise the sample is rejected. This process allows the posterior distributions of the parameters to be estimated without a likelihood function, providing a likelihood-free method of Bayesian parameter estimation. Furthermore, ABC can be combined with transdimensional Markov Chain Monte Carlo (MCMC) methods, which also propose transitions between model spaces, and provide an estimate of the Bayes factor based on the relative time spent in each model space (Green, 1995; Carlin \& Chib, 1995; Pritchard et al., 1999). Recent advancements have also combined ABC methods with neural networks, which attempt to provide efficient methods of estimating the posterior distribution by learning the summary statistics of simulated data from parameter values across the range of the parameter space, and using this to efficiently sample from the posterior distribution (Lueckmann, Bassetto, Karaletsos, \& Macke, 2018) or directly estimate the early moments of the posterior distribution (Radev, Mertens, Voss, \& Köthe, 2019). 
However, the principles underlying ABC methods are reliant on sufficient statistics: that is, a summary statistic, or set of summary statistics, which are sufficient to capture all of the information contained within the data. In many situations this is a problematic assumption, as a small number of summary statistics are insufficient to capture the information contained within the specific type of empirical data being investigated, such as the genetics data that ABC is commonly applied to (Robert et al., 2011), and the response time data in our current study. Applying ABC methods when this assumption is violated can also have practical consequences, where using a summary statistic that lacks sufficiency results in some unknown loss function of information, which can result in misleading acceptance behaviour between model spaces in transdimensional MCMC, and therefore, a misleading estimate of the Bayes factor (Robert et al., 2011).

PDA methods replace the likelihood with a pseudo-likelihood, with the pseudolikelihood being an approximation to the likelihood obtained through simulation and density estimation (Turner \& Sederberg, 2014; Holmes, 2015; Evans, Holmes, \& Trueblood, 2019). This typically involves simulating a large number of instances (e.g., decision-making trials) from the model to create model predictions, using these predictions to create a likelihood function through a method of density estimation, and then obtaining the likelihood of each observed data point under this constructed pseudo-likelihood function. Importantly, PDA methods remove the need for sufficient statistics, as an estimate of the likelihood can be obtained for each data point. PDA methods were originally introduced to psychology research by both Turner and Sederberg (2014) and Holmes (2015), who proposed creating the pseudo-likelihood function through non-parametric kernel density estimation, which estimates a large number of discrete density points across a continuous function according to a specified smoothing bandwidth (e.g., Silverman's rule of thumb; Silverman, 1986) and kernel (e.g., Epanechnikov, Gaussian), and obtaining the likelihood for each data point 
using linear interpolation, which takes the likelihoods at the two closest estimated density points and uses a linear function to infer the likelihood at the location of the observed data point. Previous studies within cognitive psychology have already applied PDA for Bayesian parameter estimation (e.g., Evans, Holmes, \& Trueblood, 2019), and our study provides a key advancement by combining PDA with TIDE to create a likelihood-free method of inference through Bayes factors, which does not contain the potentially problematic assumption of sufficient statistics that transdimensional ABC methods of Bayes factor estimation rely on.

More generally, an overarching limitation of simulation-based methods is the noise present in the simulation process, which leads to non-deterministic estimates of summary statistics or pseudo-likelihoods. Although this issue can be mitigated by using a large number of simulations, as the noise reflected in the overall distribution of simulations decreases as the number of simulations increase, increasing the number of simulations also increases the computational burden, which eventually results in the simulation-based method becoming computationally intractable (see Holmes, 2015 for a discussion). Therefore, researchers must strike a careful balance between speed and precision, in order to obtain an accurate distribution of predictions from the model within a reasonable time frame; a careful balance that often still results in noise being present, which may cause approximation error. Our current study takes several precautions to ensure that our inferences are not the result of approximation error, using repeated, independent calculations of the marginal likelihood, and performing several robustness analyses. However, due to the greater potential for approximation error in simulation-based methods, we wish to make the general recommendation that simulation-based methods should not be considered a first-step for comparing models, and instead should be considered a last resort. Ideally, researchers should use analytically or numerically computed likelihood likelihood functions in cases where they 
are available and computationally tractable. However, we believe that many psychologically interesting models result in situations where computing these deterministic likelihood functions are not possible or feasible, and that our pseudo-likelihood Bayes factors provide a principled, robust method of model comparison in these situations.

\section{TIDE and marginal likelihood estimation methods}

Thermodynamic Integration via Differential Evolution (TIDE; Evans \& Annis, 2019) is a method for estimating the marginal likelihood for a model in a specified data set. In order to determine which of a series of models provides the best explanation of the underlying psychological process, these models must be compared; however, simply assessing which model provides the best fit to the data is inadequate, as more flexible models are provided with an unfair advantage over simpler models. Previous research has suggested that the Bayes factor provides the optimal balance between goodness-of-fit and flexibility in model selection, with the Bayes factor being the ratio of the marginal likelihoods of two models (Kass \& Raftery, 1995; Myung \& Pitt, 1997; Evans \& Brown, 2018; Evans, Howard, et al., 2017). Specifically, calculating the marginal likelihood involves integrating the unnormalized posterior probability (i.e., $P(D \mid \boldsymbol{\theta}, M) P(\boldsymbol{\theta} \mid M))$ over the entire parameter space, which provides a natural punishment for flexibility that accounts for the models functional form. Models that are able to predict a broad range of data patterns a-priori based on their parameters, the prior distributions on their parameters, and their functional form - will be more heavily penalized by this integration than models that make more constrained predictions about the data patterns. However, calculating the marginal likelihood in complex models with multiple parameters requires the estimation of a multi-dimensional integral, which can be computationally intractable, and has largely restricted the use of Bayes factors to simple statistical models where the marginal likelihood can be calculated analytically. 
Recent research has focused on developing methods for estimating this multidimensional integral in complex cognitive models, through a means of either computing technology (Evans \& Brown, 2018), sophisticated sampling algorithms (Gronau et al., 2017), or mathematical identities that change the integral to a single-dimensional estimate (Annis et al., 2019). Although several potential methods exist for marginal likelihood estimation, our study focuses on Thermodynamic Integration (TI), a method from the latter category that has been applied to evidence accumulation models in several previous studies (Annis et al., 2019; Evans \& Annis, 2019; Evans, Bennett, \& Brown, 2018; Evans, 2019a). Specifically, TI involves the estimation of several "power posteriors", which are posterior distributions, but with the likelihood function of the model (i.e., the probability of the data given the parameters; $P(D \mid \boldsymbol{\theta}, M))$ raised to a power between 0 and 1 , known as temperatures. As shown by Lartillot and Philippe (2006) and Friel and Pettitt (2008), integrating the posterior mean log-likelihood over the dimension of temperature between the values of 0 and 1 will provide the marginal likelihood, given that the number of temperatures used is adequate to provide an accurate estimate of this integral. Therefore, TI requires MCMC sampling at a range of temperatures to obtain the posterior mean log-likelihood for each, which is then integrated over temperature using a simple integration rule (e.g., the trapezoidal rule) to obtain an estimate of the marginal likelihood. More details about the specifics of TI and the mathematical definitions can be found in Annis et al. (2019). However, TI (and other marginal likelihood estimation methods) still come with a sizeable computational burden, meaning that previous applications have been restricted to models with analytic likelihood functions.

One practical limitation of TI is that a large number of posterior distributions (approximately 40; Annis et al., 2019) needs to be estimated to obtain an accurate approximation of the log-marginal likelihood. TIDE attempts to reduce the practical limitation 
in TI of having to estimate a large number of independent posterior distributions by combining TI with the posterior estimation method of Differential Evolution Markov chain Monte Carlo (DE-MCMC; Ter Braak, 2006; Turner, Sederberg, Brown, \& Steyvers, 2013). DE-MCMC is a form of population MCMC, where a series of parallel chains each samples from the posterior, while interacting by informing the proposal jumps of one another. Specifically, DE-MCMC uses the difference between the parameter values of two randomly selected chains to create the proposal jump of a third chain:

$$
\boldsymbol{\theta}_{i, c}=\boldsymbol{\theta}_{i-1, c}+\gamma\left(\boldsymbol{\theta}_{i-1, l}-\boldsymbol{\theta}_{i-1, m}\right)+\epsilon
$$

where $i$ indexes the sampling iteration, $c$ is the chain to be updated, $l$ and $m$ are randomly chosen other chains, $\gamma$ is a tuning parameter that controls the size of proposal jump, and $\epsilon$ is a random variable based on a tuning parameter that adds a small amount of uniform noise. TIDE combines TI and DE-MCMC by using a series of parallel chains that interact via the DE-MCMC proposal jumps, which each estimate the posterior of a different temperature, allowing the marginal likelihood to be estimated in a single MCMC estimation procedure. Similar to DE-MCMC, it is important that TIDE is implemented with at least $2 k$ parallel chains, where $k$ is the number of free parameters in the largest updating block, to allow the chains to adequately move through the parameter space.

\section{Model comparison}

Our study aims to provide insight into two important theoretical questions regarding the flanker task. Firstly, and most notably, we assess which of the three conflict diffusion models provide the best explanation of the cognitive process underlying the flanker task. Secondly, we attempt to understand why some models provide a better explanation of the underlying process than others. 
To gain insight into which models provide the best explanation of the cognitive process underlying the flanker task, we used pseudo-likelihood Bayes factors ${ }^{1}$ to compare the 6 different models (simple and full versions of DSTP, SSP, and DMC). We only include the full diffusion comparisons within the main text for ease of communication, as a full diffusion version was found to be the superior model for every participant in every data set. We chose the Bayes factor as it closely aligns with our goal of attempting to find the model that provides the best account of the underlying process (Evans, 2019a, 2019c; Gronau \& Wagenmakers, 2019). It should also be noted that many different methods - based on different criteria - exist for selecting between competing theoretical models (Evans, 2019a), such as cross-validation methods that select models based on their ability to predict unseen data (Browne, 2000). However, cross-validation methods have been shown to lack the statistical property of consistency (Gronau \& Wagenmakers, 2019), where they fail to show increasing evidence for a data-generating simpler model with increasing amounts of data (i.e., a bias towards more complex models; Evans, 2019a), meaning that crossvalidation methods appear to be inappropriate for selecting the model that provides the best account of the underlying process. Regardless, the general concept of applying multiple model selection methods can be a useful robustness check, as the agreement of different methods increases the certainty that the choice of model does not depend on the specific assumptions of the model selection method. Although our study focuses on Bayes factors, as we believe that they are the most appropriate method for our goal, we do not wish to discourage researchers from applying other (or multiple) model selection methods, and future research should further explore which methods are most useful in which situations.

To calculate the Bayes factors, we used the recently developed method TIDE (Evans \& Annis, 2019) to approximate the log-marginal likelihood for each model. We implemented

\footnotetext{
${ }^{1}$ Note that in all cases we report the log-Bayes factor and log-marginal likelihood.
} 
TIDE with 40 parallel chains, each estimating the posterior of a different temperature, with the first 2,000 samples per chain discarded as burn-in, and then 5,000 samples taken per chain for use in TI. The approximated log-marginal likelihoods for all models can be found in the Supplementary Materials.

For the estimation of the posterior of each temperature, we used the following prior distributions: 
All models :

$$
\begin{aligned}
z & \sim T N(0.5,0.5,0,1) \\
t e r & \sim N(0.3,0.3,0, \infty) \\
a & \sim N(0.2,0.2,0, \infty)
\end{aligned}
$$

All complex variants :

$$
\begin{aligned}
& s_{t e r}, s_{z} \sim T N(0.1,0.1,0, \infty) \\
& s_{v} \sim T N(0.2,0.2,0, \infty) \\
& D S T P \text { : } \\
& a_{s s} \sim T N(0.2,0.2,0, \infty) \\
& v_{t a} \sim N(0.3,0.3) \\
& v_{f l} \sim N(0.2,0.2) \\
& v_{s s} \sim N(0.4,0.4) \\
& v_{p 2} \sim N(1.2,1.2) \\
& S S P \text { : } \\
& p \sim \operatorname{TN}(0.5,0.5,0, \infty) \\
& S D_{0} \sim T N(4,4,0, \infty) \\
& S D_{r} \sim T N(70,30,0, \infty) \\
& D M C \text { : } \\
& v_{c} \sim N(0.3,0.3) \\
& \zeta \sim T N(30,30,0, \infty) \\
& \alpha \sim T N(3,3,1, \infty) \\
& \tau \sim T N(100,100,0, \infty)
\end{aligned}
$$


where $N$ is the normal distribution with parameters $(\mu, \sigma)$, and $T N$ is the truncated normal distribution with parameters ( $\mu, \sigma$, lower bound, upper bound). The priors for the general diffusion model parameters were chosen based upon those that have been used in previous Bayesian applications of the diffusion model (e.g., Evans \& Brown, 2017; Evans, Bennett, \& Brown, 2018). The priors for the specific parameters of each conflict model were loosely based upon the ranges of generating values used in White et al. (2018), though we made these priors more uninformative due to the limited previous research on the empirical ranges of values for these parameters, and the issues found by White et al. (2018) in identifying the values of the individual parameters. However, to ensure that our choice of priors did not influence our results, we also performed an empirical Bayes robustness analysis, and our key conclusions remained unchanged (see the Supplementary Materials). Our reason for computing Bayes factors individually for each participant in each study, rather than hierarchically over all participants (e.g., Annis et al., 2019; Evans \& Annis, 2019), was to help assess the generalizability of any inferences about the models across people (see Evans, Bennett, \& Brown, 2018 for an example of how there can be large individual differences in Bayes factors and models selected between people). However, it should also be noted that several hierarchical methods have been developed that allow for differences between people in the model selected, which estimate the proportion of people who belong to each model (Stephan, Penny, Daunizeau, Moran, \& Friston, 2009; Rigoux, Stephan, Friston, \& Daunizeau, 2014), meaning that performing a hierarchical analysis does not require the assumption that all people follow the same model.

Due to each model having a computationally intractable likelihood function, we used probability density approximation (PDA; Turner \& Sederberg, 2014; Holmes, 2015) to approximate the likelihood function of each model through simulation. We simulated 10,000 trials per pseudo-likelihood function for each model, using the simulation method of 
Evans (2019b), and re-calculated the likelihood for the current parameters every 5 iterations to ensure that the variability between simulations had minimal effects on the estimated posteriors. We simulated each of the models in seconds using Euler's method (e.g., Brown, Ratcliff, \& Smith, 2006) with a time-step of 0.01 (though see the Supplementary Materials for a robustness analysis with a time-step of 0.001, where the results are unchanged), which for most participants was approximately the size of the bandwidth of the Gaussian density kernel calculated by Silverman's rule of thumb (Silverman, 1986; recommended by Holmes, 2015). It should also be noted that large time-steps (relative to the range of response times) can result in inaccuracies (Brown et al., 2006), and care should be taken when simulating using Euler's method to ensure that the choice of time-step does not impact upon the results.

As our assessment involved three levels of approximation/estimation - marginal likelihood approximation with TI, posterior estimation with DE-MCMC, and likelihood function approximation with PDA - we estimated the marginal likelihood for each model for each person 5 independent times. When comparing models through log-Bayes factors (i.e., the natural logarithm of the Bayes factor), the superior model was decided by whichever model had the maximum log-marginal likelihood over all 5 approximations. However, the log-Bayes factor was obtained by taking the minimum log-marginal likelihood of the superior model, and subtracting it by the maximum log-marginal likelihood of the inferior model, meaning that our log-Bayes factors reflected the minimum difference between the models. Therefore, if there were variability in the approximated log-marginal likelihoods that was large enough to potentially affect which model was selected as the superior model, then the log-Bayes factors that we calculated would provide negative values. In contrast, positive log-Bayes factors would be indicative of systematic differences between the models far the noise in the approximations. For positive log-Bayes factors, we interpreted the 
strength of evidence for the winning model in line with Jeffreys (1961), which can be seen in Table 1.

Table 1: Displays our interpretations of the strength of evidence in favour of a model based upon the log-Bayes factor. Note that our wording of the interpretations differ slightly from those of Jeffrey's (1961), as we believe "weak" and "moderate" more clearly and intuitive categorize the strength of evidence across increasing Bayes factors.

\begin{tabular}{lcc}
$\log _{e}(\mathrm{BF})$ & Our interpretation & Jeffrey's (1961) interpretation \\
\hline 0 to 1.16 & Weak & Not worth more than a bare mention \\
1.16 to 2.3 & Moderate & Substantial \\
2.3 to 4.61 & Strong & Strong \\
$>4.61$ & Decisive & Decisive \\
\hline
\end{tabular}

To gain insight into why some models provide a better explanation of the underlying process than others, we used a series of qualitative visual assessments that are common within the response time modelling and conflict task literature. These assessments each require the parameter values to be estimated for each model, which we obtained through Bayesian hierarchical estimation of the posterior distributions (Shiffrin, Lee, Kim, \& Wagenmakers, 2008; see Evans, Rae, Bushmakin, Rubin, \& Brown, 2017; Evans, Steyvers, \& Brown, 2018; Evans, Brown, Mewhort, \& Heathcote, 2018 for applications). Bayesian hierarchical estimation constrains the parameter estimates of each individual to follow a group-level distribution for each parameter, allowing the data of different individuals to mutually inform the parameter estimation of one another. Specifically, each group level distribution was either defined as normal or truncated normal, with the priors on the mean parameter being the same as those used in the TIDE marginal likelihood approximation for each parameter. The prior on the standard deviations was a gamma distribution with a shape and scale of 1 , with gamma distributions being commonly used for the priors on group-level standard deviations (Evans, Brown, et al., 2018; Evans \& Brown, 2017; Evans, Steyvers, \& Brown, 2018). The posterior means for these estimated parameters can be 
seen in the Supplementary Materials.

Once the posterior distributions had been estimated, we took the posterior mean of each parameter value for each model for each participant, and used these to generated predicted data from the model. We then contrasted these predictions to the observed data using several qualitative visual assessments: defective cumulative density function (DCDF) plots, conditional accuracy function (CAF; Gratton, Coles, Sirevaag, Eriksen, \& Donchin, 1988 ) plots, error location index (ELI; Servant, Gajdos, \& Davranche, 2018) plots, and delta function (DF; De Jong, Liang, \& Lauber, 1994; W. Schwarz \& Miller, 2012) plots. DCDF plots show the response time distributions for correct responses and different experimental conditions, plotted as the cumulative density (y-axis) at selected response time quantiles (x-axis). Cumulative densities are then weighted by the proportion of correct trials, allowing misfit in accuracy and misfit across each part of the response time distribution to be simultaneously assessed. CAF plots are constructed by sorting the response time data into $x$ bins of equal size, and plotting the accuracy (y-axis) against mean response time (x-axis) in each bin. CAF plots thus allow misfit in accuracy across each part of the response time distribution to be visually assessed. ELI plots provides a simple metric for the relative speed of correct and error responses in incompatible trials, derived from the error location function. While CAF shows the proportion of errors in a given response time bin (calculated with respect to the number of trials in the bin), the error location function shows the proportion of all errors below each response time quantile, and does not require data binning (for mathematical details, see Servant, Gajdos, \& Davranche, 2018). ELI is simply the area under the error location function; it is bounded between 0 and 1 , where a value of 0 indicates that all error trials are slower than all correct trials, and a value of 1 indicates that all error trials are faster than all correct trials. DF plots are constructed by plotting the difference (y-axis) against the average (x-axis) of 
equivalent correct response time quantiles between incompatible and compatible conditions, allowing misfit in the time-course of the flanker compatibility effect to be visually assessed. These analyses intend to provide insight into what qualitative aspects of the empirical data different models are successfully capturing, and what aspects the models are failing to account for, which should help to understand (1) why certain models perform better than others, and (2) where future model development may be useful to provide an even more accurate explanation of the flanker task.

\section{Results}

Determining which models provide better explanations of the flanker task

White et al. (2011). Figure 1 plots the Bayes factor analysis for the White et al. (2011) data. Each plot displays a different subject, and different colours display the estimated log-marginal likelihoods for the different classes of models. Different points of the same colour on the same point of the x-axis display the 5 different approximated logmarginal likelihoods for the model. As mentioned previously, we only include comparisons between the "full" versions of these models in the figures for brevity, as in all cases the best model was a complex version. Figures with the marginal likelihoods for all models can be seen in the Supplementary Materials, and the pattern of results were similar regardless of the incorporation of between-trial variability parameters. 

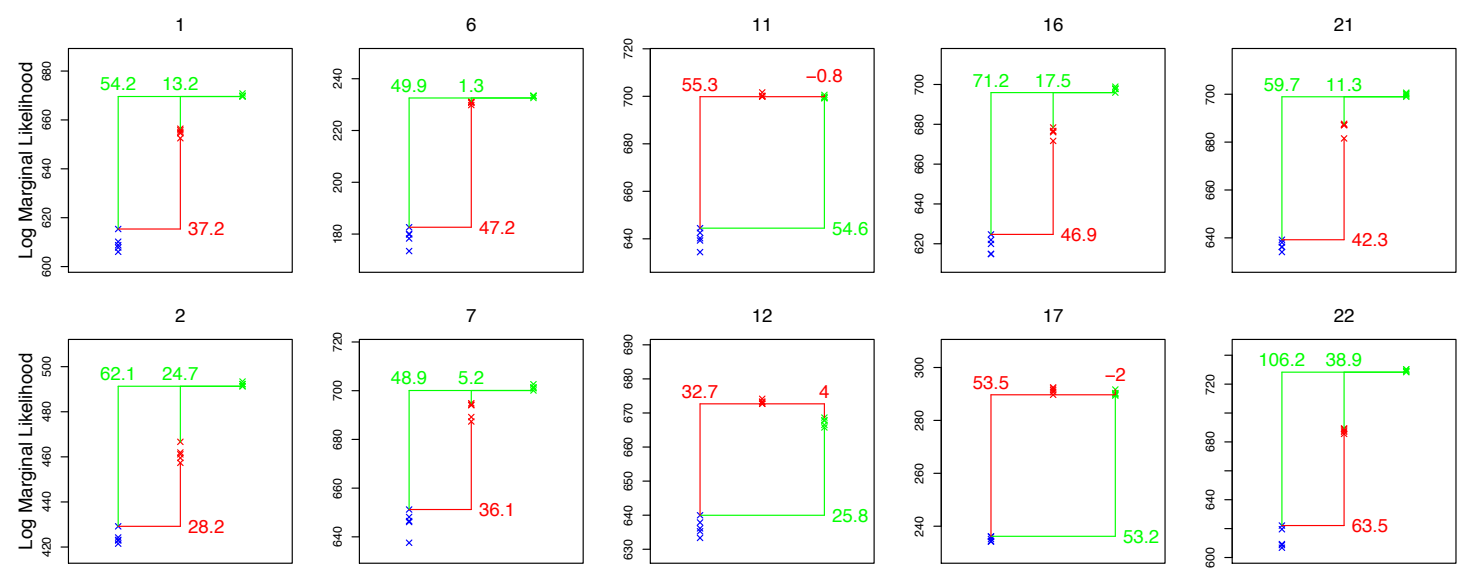

12

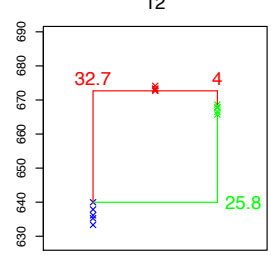

17
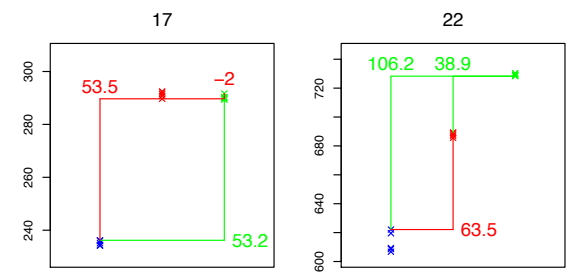

3
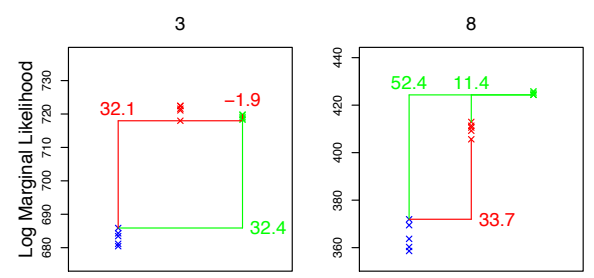

13
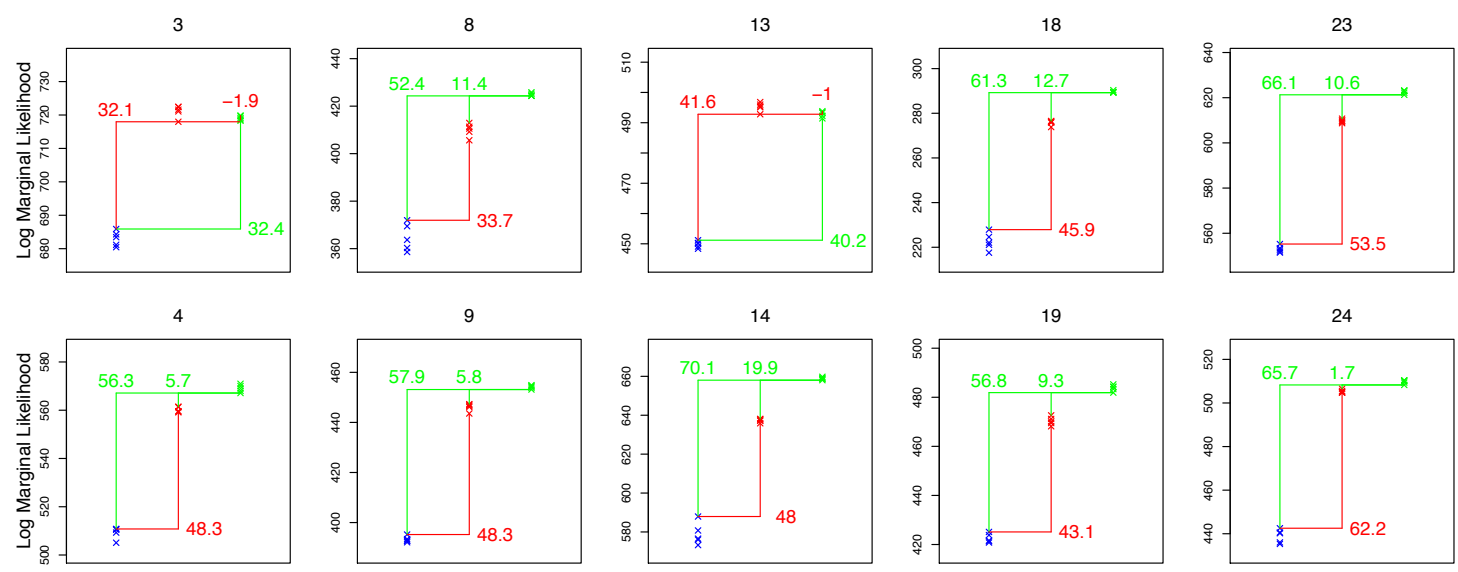

19
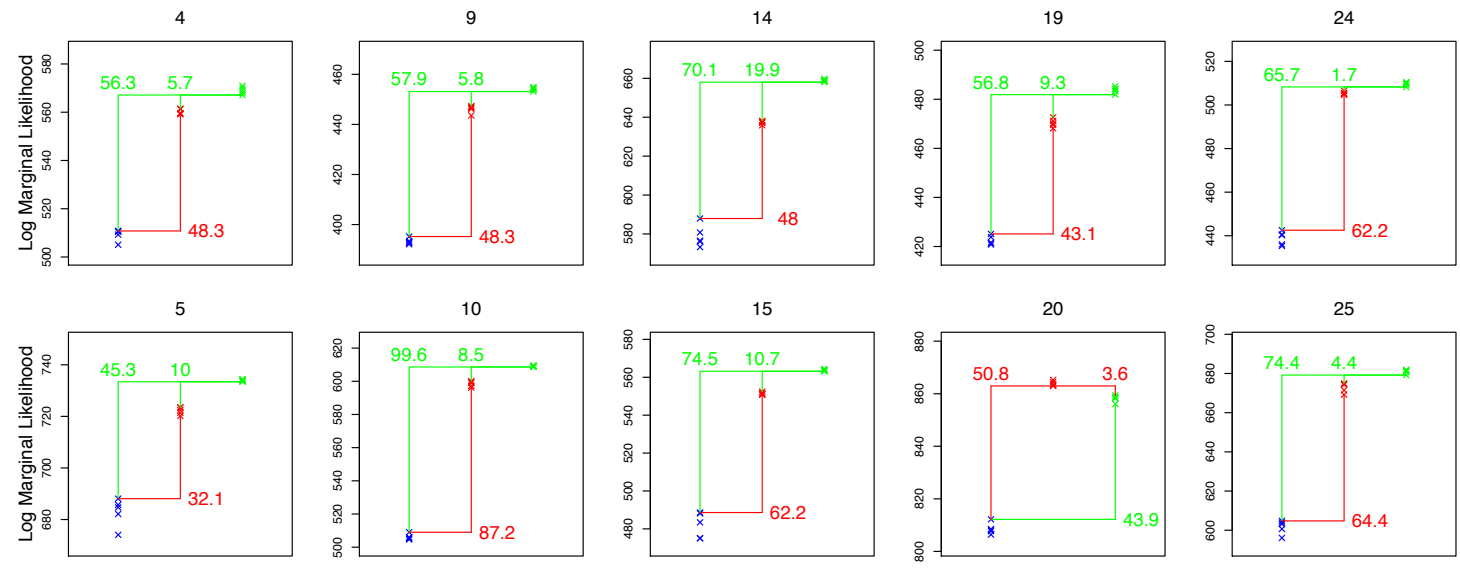

Figure 1. Displays the log marginal likelihoods for DMC (blue crosses), DSTP (red crosses), and SSP (green crosses), across 5 different estimation runs (different crosses), for each subject (different plots) in the data of White et al. (2011). Note that the x-axis displays different models. Log-Bayes factors are displayed for comparisons between these models, which are calculated as discussed in the main text. Connecting lines show the marginal likelihood estimates being compared, with the colour of the line and written log-Bayes factor indicating the winning model in the comparison. All models shown are the complex versions, with equivalent plots including the simple versions being shown in the Supplementary Materials. 
First and foremost, DMC performs systematically worse than DSTP and SSP for every participant in these data. When looking at the log-Bayes factor analysis, SSP ranges from 25.8 to 106.2 in log-Bayes when compared against DMC, meaning that in all cases the data are at least $1.6 \times 10^{11}$ more likely under SSP than DMC. DSTP shows a similar range of log-Bayes factor when compared to DMC, ranging from 28.2 to 87.2, meaning that in all cases the data are at least $1.77 \times 10^{12}$ more likely under DSTP than DMC. These analyses provide conclusive evidence that DMC provides a worse account of the White et al. (2011) data than both DSTP and SSP, and that this inferiority is true for all participants within the study.

When comparing the ability of DSTP and SSP to account for these data, the results are less conclusive, though appear to show a general preference for SSP. Of the 25 subjects, 4 show no clear evidence for either model (log-Bayes factor $<0$ ), 19 show evidence for SSP, and 2 show evidence for DSTP. Although both participants who favour DSTP show strong evidence in favour of DSTP (log-Bayes factors of 3.6 and 4), 17 of the participants who favour SSP show at least strong evidence in favour of SSP, and 16 of those participants show decisive evidence. Therefore, SSP appears to provide a better account of the data from White et al. (2011) than DSTP, though not to the same extent that SSP and DSTP outperform DMC.

Ulrich et al. (2015). Figure 2 plots the Bayes factor analysis for the Ulrich et al. (2015) data. Interestingly, the trends within these data appear to be less conclusive than the data of White et al. (2011), with all models performing more similarly. Importantly though, DMC is again found to be the worst model for every participant, with the winning model always being strongly preferred over DMC. However, the $2^{\text {nd }}$ best model is not always strongly preferred over DMC, with one participant (14) showing unclear evidence between DSTP and DMC (log-Bayes factor $=-1.9)$, and another three participants showing only 
moderate evidence. However, there is still at least strong evidence that DMC is the worst model in 12/16 participants, and that DMC is not the best model in all 16 participants. 
1

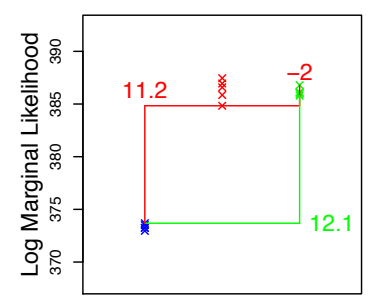

2

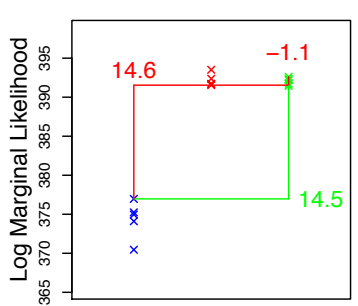

3

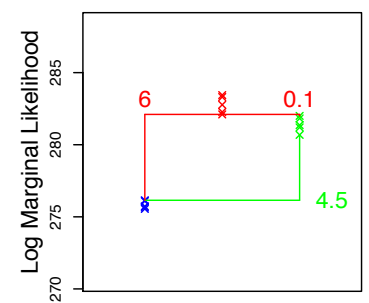

4

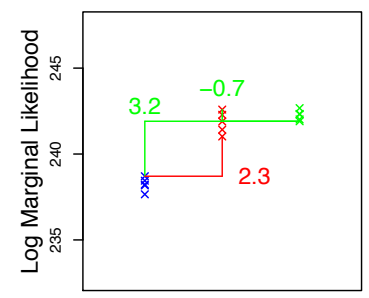

5

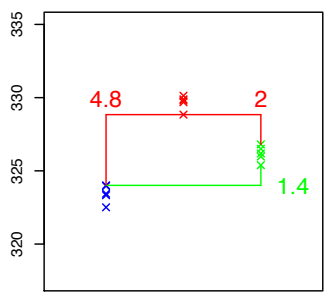

6

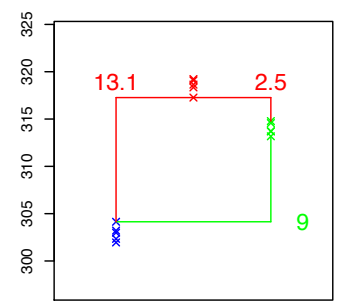

7

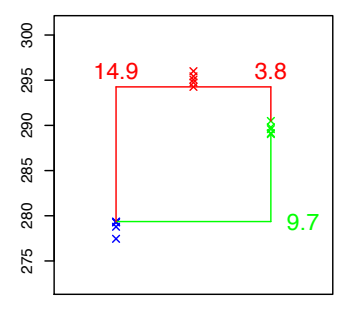

8

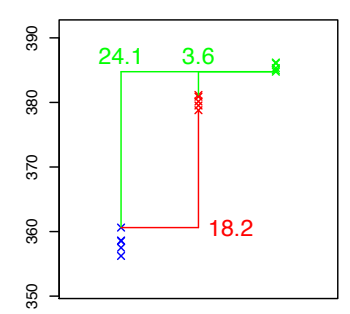

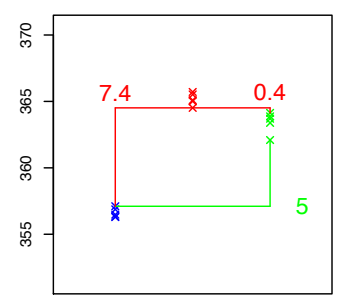

10

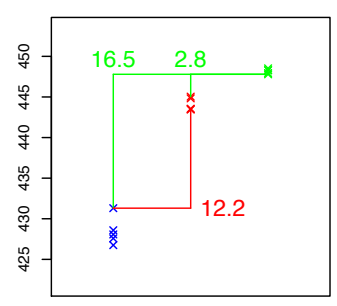

11

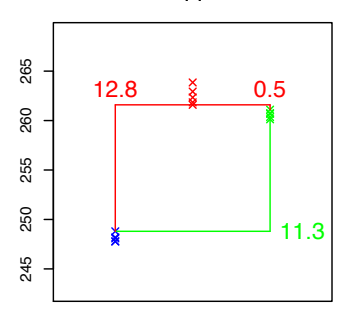

12

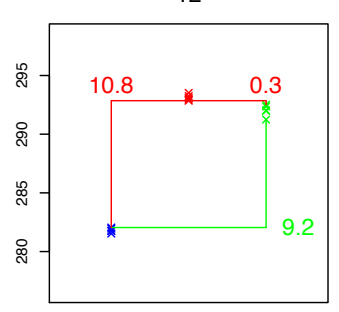

13

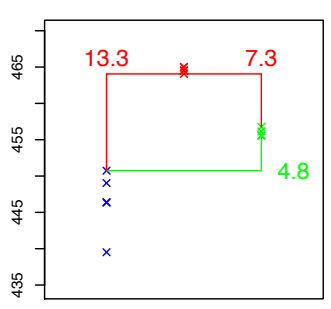

14

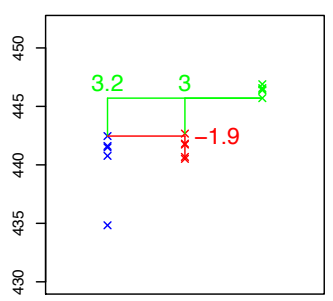

15

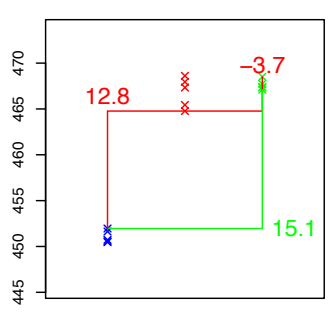

16

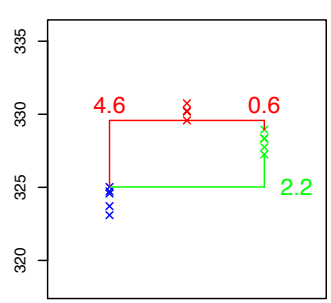

Figure 2. Displays the log marginal likelihoods for DMC (blue crosses), DSTP (red crosses), and SSP (green crosses), across 5 different estimation runs (different crosses), for each subject (different plots) in the data of Ulrich et al. (2015). Note that the x-axis displays different models. Log-Bayes factors are displayed for comparisons between these models, which are calculated as discussed in the main text. Connecting lines show the marginal likelihood estimates being compared, with the colour of the line and written log-Bayes factor indicating the winning model in the comparison. All models shown are the complex versions, with equivalent plots including the simple versions being shown in the Supplementary Materials. 
The comparison between DSTP and SSP for the Ulrich et al. (2015) data yields mixed results, with many participants showing a limited strength of evidence in either direction. Four participants show no clear evidence for either model (log-Bayes factors < 0), five participants show weak evidence in favour of DSTP, one participant shows moderate evidence, and three participants show strong or decisive evidence, with the remaining three participants showing strong evidence in favour of SSP. Although the evidence is much weaker in these data than those from White et al. (2011), DSTP seems to provide a slightly better account of the Ulrich et al. (2015) data than SSP.

Servant et al. (2015). Figure 3 plots the Bayes factor analysis for the Servant et al. (2015) data. Like the White et al. (2011) data, there appears to be more conclusive differences between the models, with stronger evidence than the Ulrich et al. (2015) data. Importantly, DMC performs worse than DSTP and SSP for every participant in the Servant et al. (2015) data, which has been the case in all three data sets that we have assessed. The log-Bayes factor for SSP compared to DMC ranges from 14.8 to 58.6, and for DSTP compared to DMC ranges from 6.4 to 65.8 , meaning that for all participants there is decisive evidence for the superiority of DSTP and SSP over DMC. 

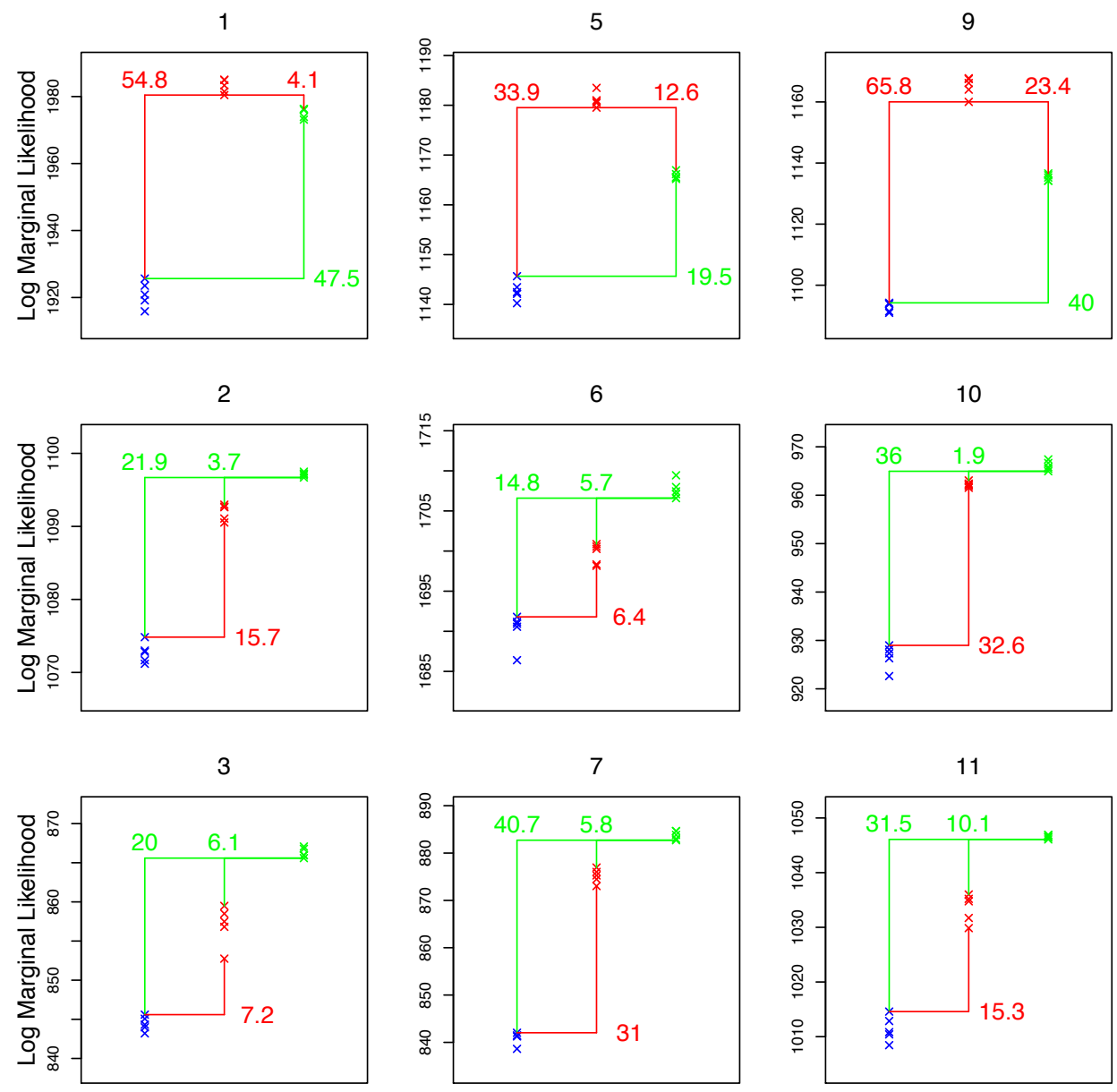

4
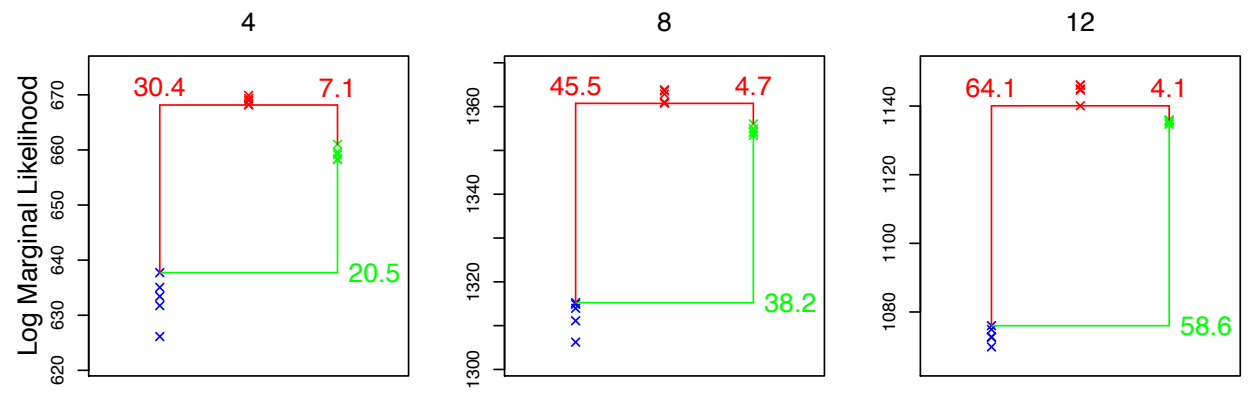

Figure 3. Displays the log marginal likelihoods for DMC (blue crosses), DSTP (red crosses), and SSP (green crosses), across 5 different estimation runs (different crosses), for each subject (different plots) in the data of Servant et al. (2015). Note that the x-axis displays different models. Log-Bayes factors are displayed for comparisons between these models, which are calculated as discussed in the main text. Connecting lines show the marginal likelihood estimates being compared, with the colour of the line and written log-Bayes factor indicating the winning model in the comparison. All models shown are the complex versions, with equivalent plots including the simple versions being shown in the Supplementary Materials. 
Like the Ulrich et al. (2015) data, the comparison between DSTP and SSP for the Servant et al. (2015) data yields mixed results, though the overall strength of evidence for each participant is stronger. Six participants show strong or decisive evidence in favour of DSTP, five participants show strong to decisive evidence in favour of SSP, and one participant shows moderate evidence in favour of SSP. These findings suggest that although there is clear evidence in most cases for what model best accounts for a specific person's data, the participants are equally split between evidence in favour of DSTP and SSP.

Understanding why DMC provides a poorer explanation of the flanker task

To try and gain some insight into why DMC provided a poorer account of these data than DSTP and SSP, we plotted the model predictions (based on the posterior mean) in combination with the empirical data to assess which trends the models were/weren't able to capture. As discussed previously, we visualized the observed and predicted data for each model using 4 different methods: DCDF plots, CAF plots, DF plots, and ELI plots.

Figure 4 shows the DCDF plots for each model (rows) in each data set (columns). For the White et al. (2011) data set there are several clear discrepancies between the observed data and the DMC predictions. Firstly, and most noticeably, DMC provides a poor fit to the later quantiles of the correct response time distribution in both the compatible and incompatible conditions, under-predicting these quantiles for compatible trials and over-predicting these quantiles for incompatible trials. Secondly, DMC under-predicts the accuracy in the incompatible condition, which can be seen in the lower response proportions in the predicted defective density than the observed defective density. Lastly, DMC underpredicts the leading edge of the response time distribution (i.e., the early quantiles) for incompatible trials, predicting that it should be much closer to that of compatible trials than is actually observed. Although the predictions of DSTP and SSP do not perfectly match the observed data, their discrepancies are much more subtle than those of DMC, 
providing an initial indication of why DMC provided an inferior explanation of the White et al. (2011) data set than DSTP and SSP. For the Ulrich et al. (2015) and Servant et al. (2015) data sets, the misfit of DMC is much more subtle, and much closer to that of DSTP and SSP. DMC again under-predicts the leading edge for incompatible trials in both data sets, though the discrepancy is reduced compared to the White et al. (2011) data set, and although DMC still provides some misfit to the later quantiles of both compatible and incompatible trials in the Servant et al. (2015) data set, this is not the case for the Ulrich et al. (2015) data set. In summary, the DCDF plots show that DMC provides a greater misfit to the data than DSTP and SSP in all data sets, though this difference in misfit ranges from large (White et al., 2011) to very minor (Ulrich et al., 2015). 

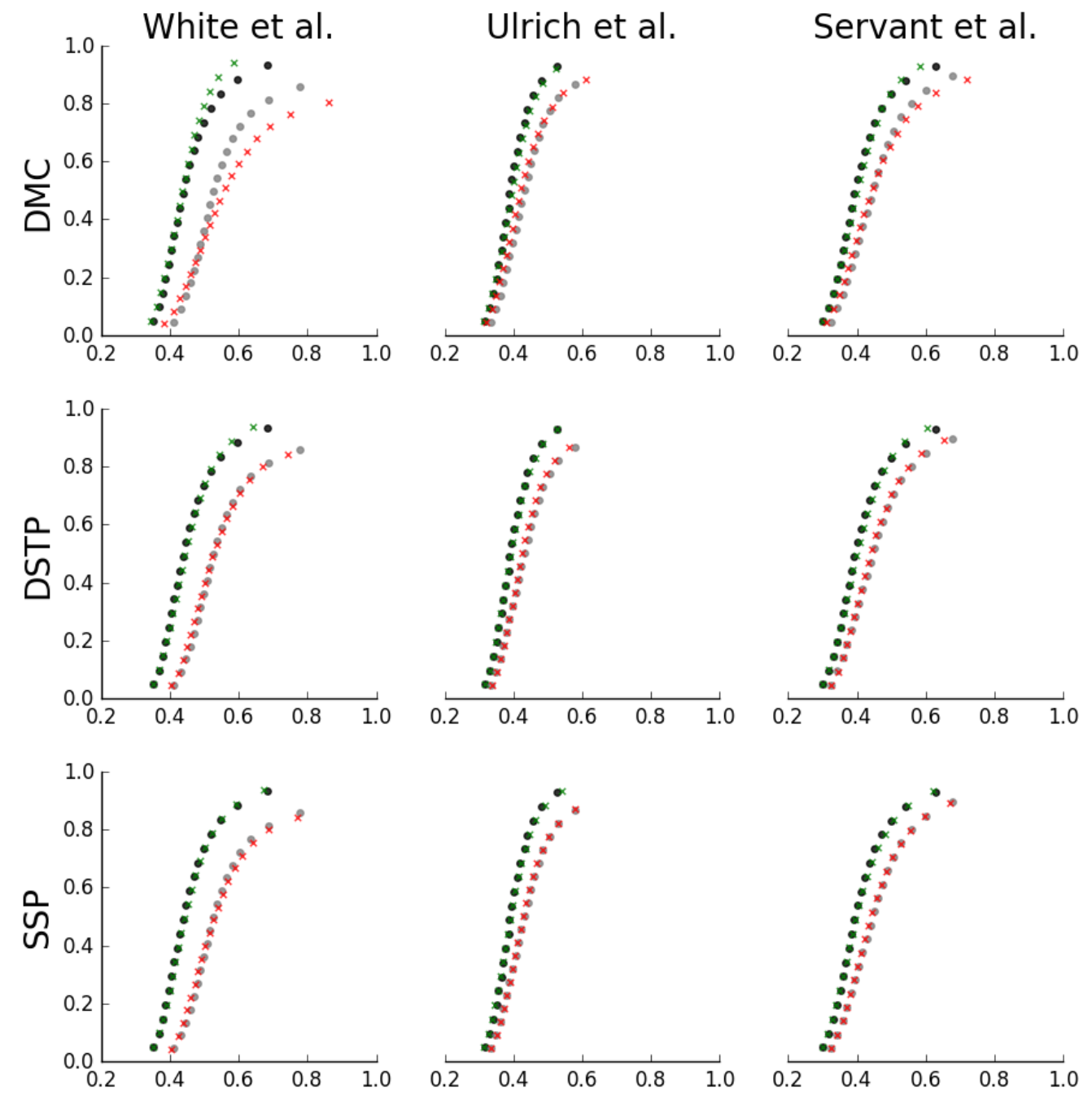

Figure 4. Defective cumulative density function (DCDF) plots for each model (rows) in each data set (columns). The DCDF plots display 19 (0.05 to 0.95, in increments of 0.05) response time quantiles (different points) of correct responses for each experimental condition, plotted by the response time (x-axis) and response proportion (y-axis) for that quantile. Dots display observed data, with black dots displaying the compatible condition and grey dots displaying the incompatible condition. Crosses display model predictions, with green crosses displaying the compatible condition and red crosses displaying the incompatible condition. 
Figure 5 shows the CAF plots for each model (rows) in each data set (columns). CAF plots from the observed data in flanker studies typically show a pattern where in compatible trials the accuracy is consistent and high across the entire response time distribution, whereas in incompatible trials the accuracy is initially low, but then increases with longer response times. All three studies show this typical pattern, though each to a different extent: the White et al. (2011) data set shows the most extreme version of this pattern with accuracy initially at chance in incompatible trials, the Ulrich et al. (2015) data set shows a moderate version with accuracy initially around $70 \%$ in incompatible trials, and the Servant et al. (2015) data set shows the most mild version with accuracy initially around $85 \%$ in incompatible trials. DSTP and SSP provide a good account of this pattern, and the different extremities of this pattern, in all three data sets, though they do display some minor misfit throughout. In contrast, DMC appears to have some difficulties capturing this pattern in all three data sets, under-predicting the accuracy with longer response times in incompatible trials. For the White et al. (2011) data set DMC under-predicts the accuracy for much of the distribution, whereas for the Ulrich et al. (2015) data set DMC initially over-predicts the accuracy, before under-predicting the accuracy for the remainder of the distribution. In summary, the CAF plots show that DMC is unable to capture the change in accuracy in incompatible trials over the response time distribution, providing an underprediction of accuracy in later parts of the distribution, and providing poor prediction in some cases under, and in other cases over - in the early parts of the distribution. 

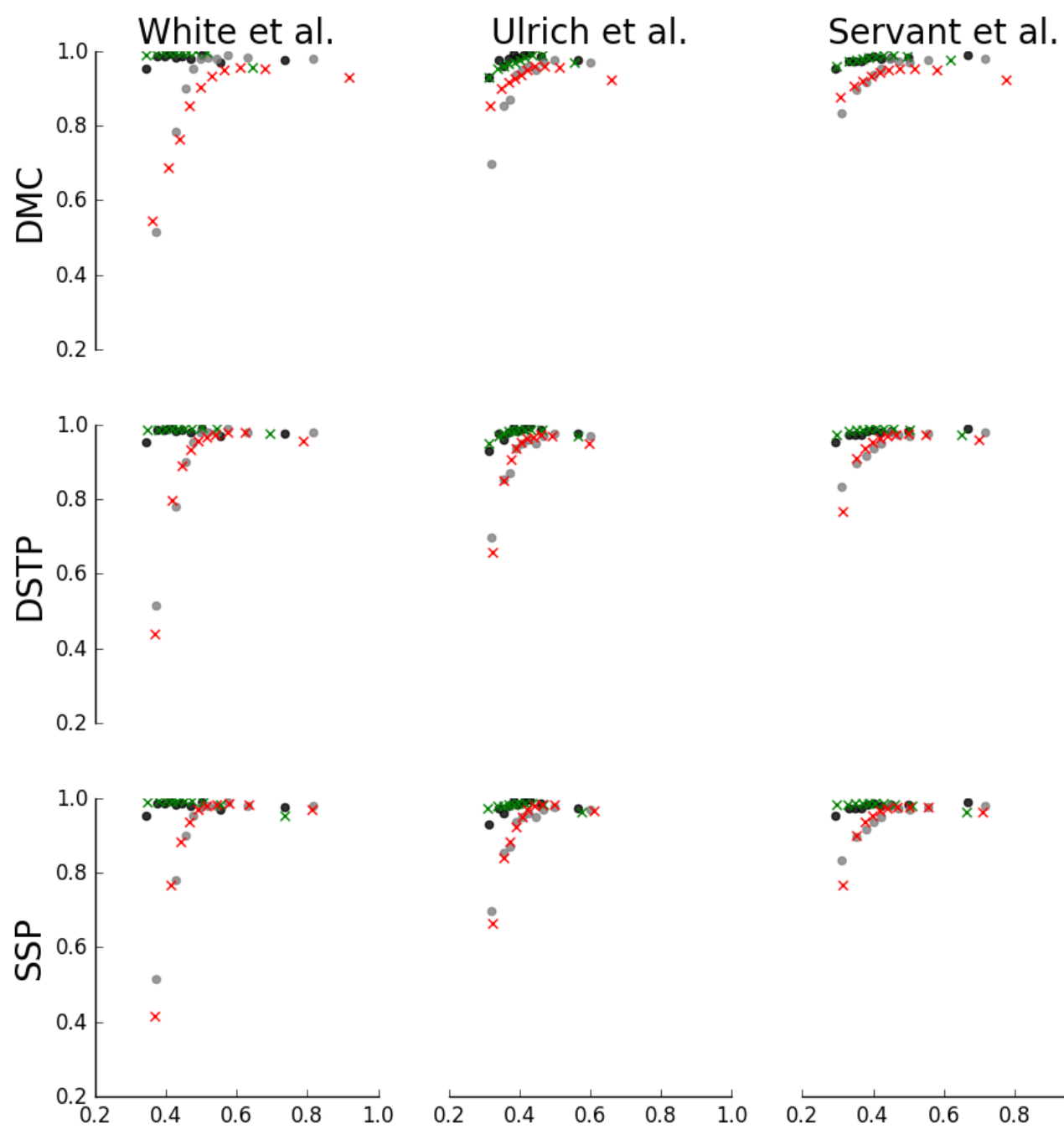

Figure 5. Conditional accuracy function (CAF) plots for each model (rows) in each data set (columns). The CAF plots display 10 response time bins (different points), with the upper limit of each bin corresponding to response time quantiles (0.1 to 1 , in increments of 0.1 ) for each experimental condition, plotted by the mean response time (x-axis) and response accuracy (y-axis) for that bin. Dots display observed data, with black dots displaying the compatible condition and grey dots displaying the incompatible condition. Crosses display model predictions, with green crosses displaying the compatible condition and red crosses displaying the incompatible condition. 
Figure 6 shows the ELI plots for each model (rows) in each data set (columns). The distribution of error response times in incompatible trials is largely faster than the distribution of correct response times in all three data sets, which is particularly the case in the (White et al., 2011) data set, where errors are highly concentrated among the fastest responses with an ELI of around 0.85. In all three data sets DMC provides a large under-prediction of the ELI, instead predicting that the distributions of error and correct response times in incompatible trials are quite similar (predicted ELI of around 0.5 in both the White et al. (2011) and Ulrich et al. (2015) data sets). In contrast, DSTP and SSP perfectly capture the ELI in both the Ulrich et al. (2015) and Servant et al. (2015) data sets, showing that they capture the characteristic pattern of fast errors in incompatible trials. Interestingly, the ELI plots also provide clear insight into why SSP provides a better explanation than DSTP for most participants in the (White et al., 2011). Specifically, DSTP under-predicts the ELI, predicting that the error and correct response times are more similar than they actually are, whereas SSP predicts this trend near perfectly. As a greater proportion of fast errors are observed in White et al. (2011) than both other data sets, the advantage of SSP over DSTP in White et al. (2011) may be due to unique aspects of the experimental paradigm (e.g., the $350 \mathrm{~ms}$ inter-trial interval, which was faster than the other studies), rather than any general superiority of SSP over DSTP. In summary, the ELI plots show that DMC is unable to capture the fast errors observed within the incompatible condition of the flanker task in all three data sets, whereas DSTP and SSP both capture this pattern well. 


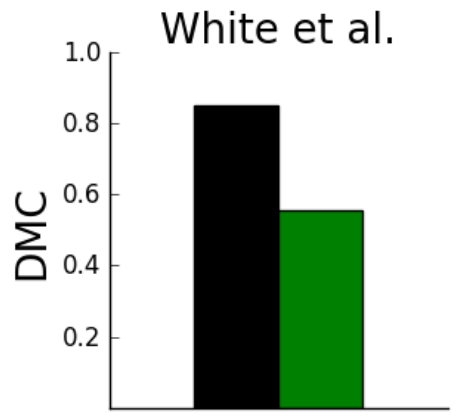

Ulrich et al.

Servant et al.
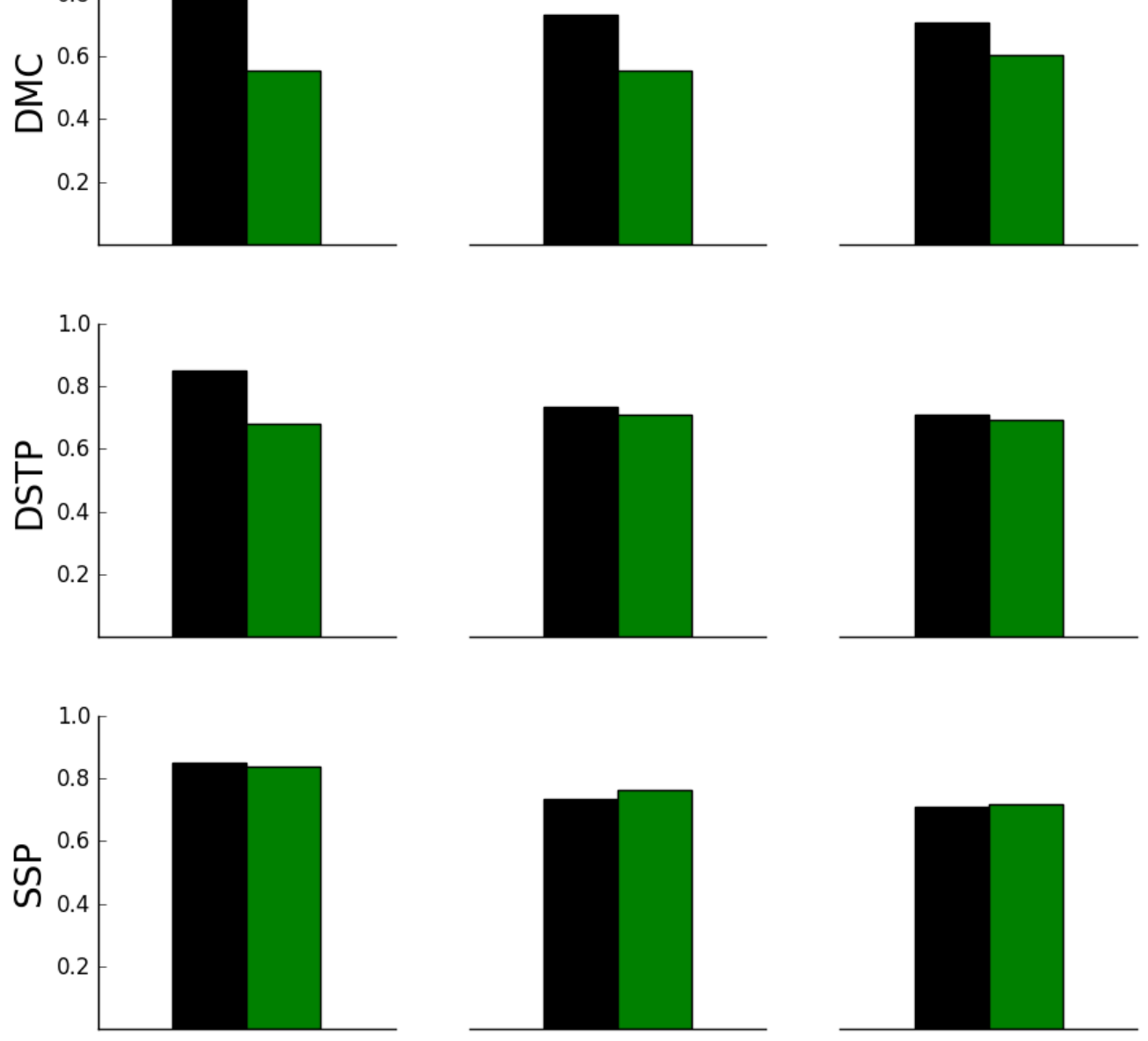

Figure 6. Error location index (ELI) plots for each model (rows) in each data set (columns). The ELI plots display the relative speed of correct and error responses in incompatible trials across the entire response time distribution, with a value of 1 indicating that all errors are concentrated among the fastest incompatible responses, and a value of 0 indicating that all errors are concentrated among the slowest incompatible responses (y-axis). Black bars display the observed data, and green bars display the model predictions. 
Figure 7 shows the DF plots for each model (rows) in each data set (columns). DF plots from the observed data show a consistent pattern where correct response times for compatible trials are faster than incompatible trials, and this difference in relative speed increases from early responses to median responses, though in later responses the difference is more stable. DSTP and SSP provide a good account of the general pattern in the DF, capturing the initial increase followed by the later stability. In contrast, DMC is unable to capture this general pattern: although DMC captures the initial increase, DMC predicts a continuing increase in the later responses, rather than the stability observed in the data. Interestingly, DSTP and SSP do not perfectly capture the DF functions, showing underprediction and over-prediction for several parts of the data, suggesting that both DSTP and SSP may still benefit from further model development. In summary, the DF plots show that DMC is unable to capture the stabilization in the difference between correct response times in compatible and incompatible trials (i.e., the flanker effect) for later responses, and instead predicts that the flanker effect continues to grow larger in later responses. 


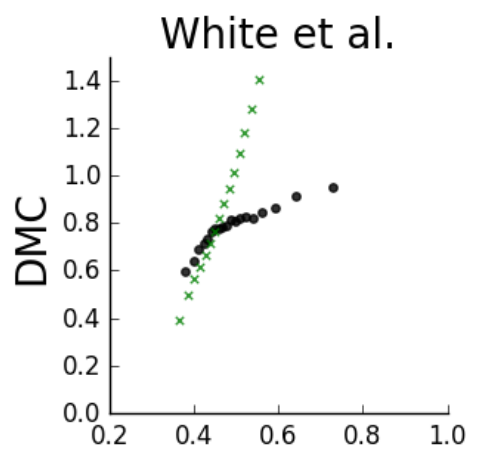

Ulrich et al.

\section{Servant et al.}
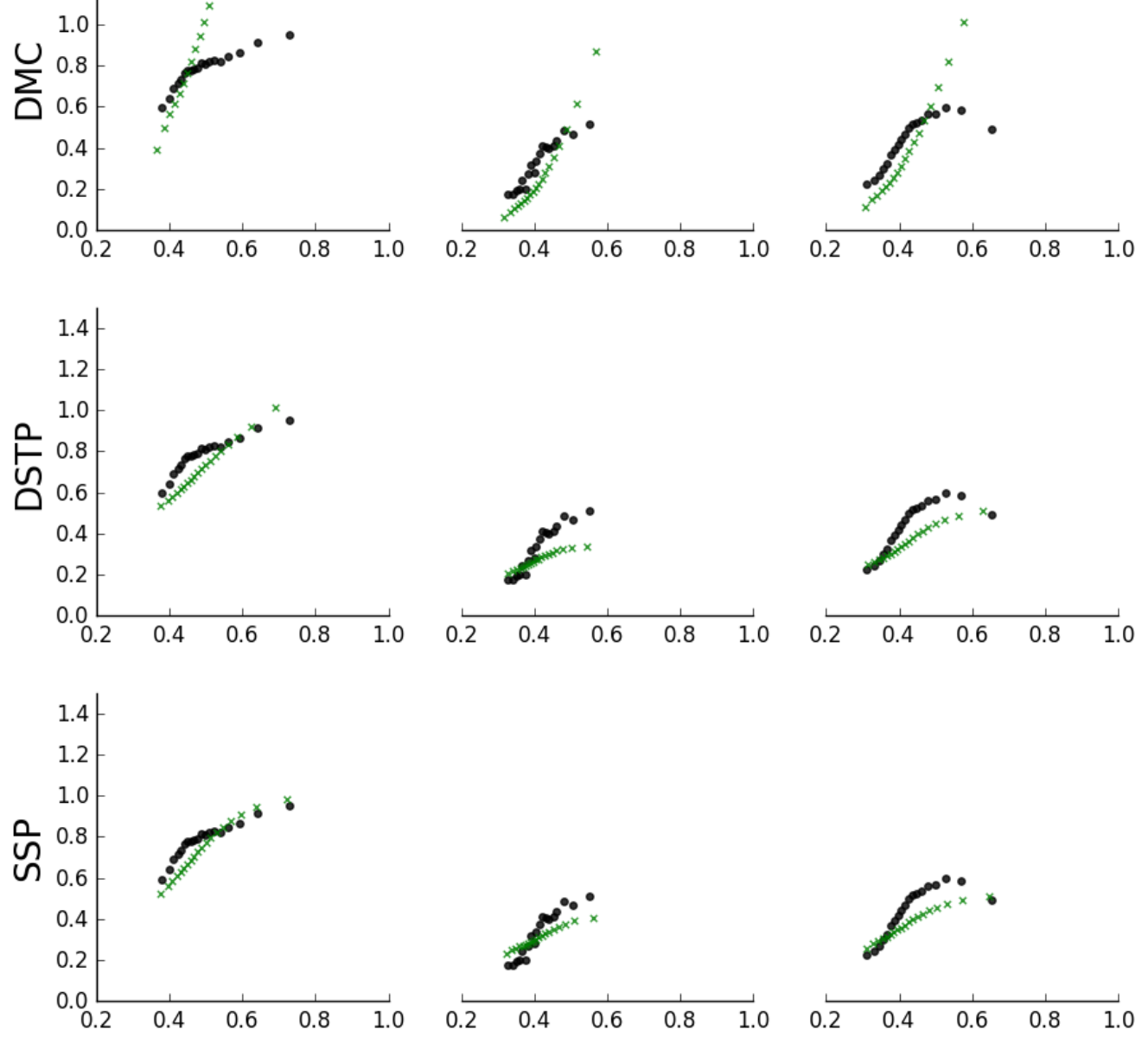

Figure 7. Delta function (DF) plots for each model (rows) in each data set (columns). The DF plots display 19 (0.05 to 0.95, in increments of 0.05) response time quantiles (different points) of correct responses for each experimental condition, plotted by the average response time across conditions (x-axis) and the difference in response time between conditions (y-axis) for that quantile. Black dots represent observed data, and green crosses represent model predictions. 
Overall, these qualitative trends suggest that DMC provides a poorer explanation of the flanker task than DSTP and SSP for several reasons: (1) under-estimation of the accuracy in later incompatible trials responses, (2) under-estimation of the response time of errors relative to correct responses, and (3) over-estimation of the difference between incompatible and compatible trial correct response time in later trials. Specifically, the visual patterns predicted by DMC all appear to point to one common issue: a continuing influence of DMC's automatic process in later stages of the decision. Theoretically, the automatic process within DMC is designed to provide an initial, automatic influence of the task-irrelevant flanker stimuli, which then decreases over time until the flankers no longer have any influence (for a review of experimental data supporting this hypothesis, see Ulrich et al., 2015 p. 152). Practically, the expected mean of the automatic activation (i.e., accumulated drift rate) is modelled as a scaled gamma function, which under many parameterizations will show this pulse-like shape. However, the incorrect predictions of DMC appear to suggest that this is not the case, and that the automatic activation is still strong during the later parts of the decision process. To assess whether this was the case, we used the posterior means (which can be seen in the Supplementary Materials) for each data set to calculate the expected mean of the automatic activation $E\left[X_{a}(t)\right]$, which corresponds to the integral of the time-varying drift rate of the automatic process:

$$
E\left[X_{a}(t)\right]=\int_{0}^{\infty} v_{a}(t) d t=\zeta \times e^{\frac{-t}{\tau}} \times\left[\frac{t \times e}{(\alpha-1) \times \tau}\right]^{(\alpha-1)}
$$

The expected mean of the automatic process averaged across subjects for each data set is shown in Figure 8. Interestingly, under these parameterisations the expected mean of the automatic activation either continues to increase (the Ulrich et al., 2015 and Servant et al., 2015 data sets) or remains stable (the White et al., 2011 data set) over the course of time that most decisions are made within (i.e., 0.8s from accumulation onset). This provides 
a clear explanation for DMC's poor account of the flanker task: the model predicts a continuous or increasing influence of flanker stimuli over the course of the decision, whereas the empirical data appear to show trends consistent with a decreasing influence of flanker stimuli. As a side note, these findings are in contrast to those of Ulrich et al. (2015), who found that DMC provided an accurate account of the CAF and DF patterns in their flanker data when fit directly to the group-averaged CAFs and correct response CDFs, using a root mean squared error (RMSE) cost function with an arbitrary weight assigned to the CAFs relative to the correct response CDFs. Importantly, the difference between our findings and those of Ulrich et al. (2015) could be due to (1) our use of the entire response time distributions, rather than the 19 quantiles for only correct responses used by Ulrich et al. (2015), (2) our use of a Bayesian approach that weights all data equally, rather the arbitrary weight between correct response CDFs and CAFs used by Ulrich et al. (2015), and/or (3) our use of fits to individual participants, rather than the fitting of grouplevel trends used by Ulrich et al. (2015) (a practice that has known averaging issues; see Estes, 1956; Heathcote, Brown, \& Mewhort, 2000; Evans, Brown, et al., 2018). However, it should be noted that previous studies have suggested that individual participant and group-level conflict diffusion model analyses lead to identical conclusions, meaning that averaging issues may not be a practical concern in the context of conflict diffusion models (Servant et al., 2016; Mahani, Bausenhart, Ahmadabadi, \& Ulrich, 2018). Interestingly, our findings suggest that DMC provides a poor account of the typical CAF and DF patterns in flanker data when constrained by the entire response time distributions for each individual participant, as it must sacrifice capturing these patterns to capture other patterns in the data. 

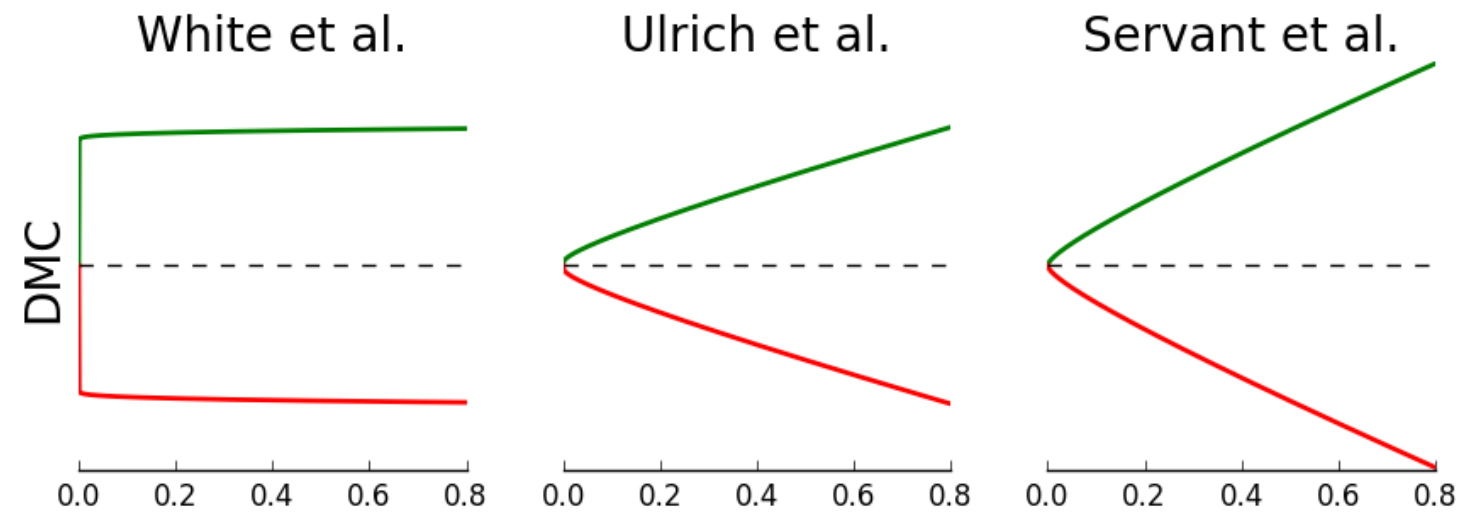

Figure 8. Plots of the time-course of the expected mean of the automatic activation $E\left[X_{a}(t)\right]$ from the DMC. The y-axis displays $E\left[X_{a}(t)\right]$, and the x-axis displays the time since accumulation onset. Green lines display the compatible condition, and red lines display the incompatible condition. Black lines indicate the unbiased starting point of accumulation, halfway between the two decision bounds.

\section{Discussion}

Our study aimed to provide the first comprehensive quantitative comparison between the three key models of the flanker task - the DSTP (Hübner et al., 2010), the SSP (White et al., 2011), and the DMC (Ulrich et al., 2015) - using the flanker data from three previous studies - White et al. (2011), Ulrich et al. (2015), and Servant et al. (2015). Each of these models have been developed as theoretical extensions of the diffusion model (Ratcliff, 1978) and provide an explanation for how interference effects occur in conflict tasks. Previous research has found that these models make quantitatively distinguishable predictions about the response time distributions, which suggests that they can be quantitatively compared (White et al., 2018). In addition, White et al. (2018) showed that the models based on theories of visual attention (DSTP and SSP) provide measures of cognitive control that 
differ from the model based on theories of automaticity (DMC). We attempted to establish which model(s) provide the best account of the flanker task, and therefore, which measure(s) of cognitive control should be trusted. Previous studies have found mixed results when quantitatively comparing "simple diffusion" variants of DSTP and SSP, and no previous studies have incorporated DMC into the comparison, which is a key limitation as DMC diverges from DSTP and SSP about inferences for cognitive control. Our study compared simple and full diffusion variants of all three models, using probability density approximation (PDA; Turner \& Sederberg, 2014; Holmes, 2015) and thermodynamic integration via differential evolution (TIDE; Evans \& Annis, 2019) to calculate Bayes factors for these models with computationally intractable likelihood functions.

\section{Theoretical impacts of our study}

Interestingly, our findings provided a clear answer to our central theoretical question: which conflict diffusion models provide a better explanation of the flanker task than others. For every participant within each of the three studies assessed (53 total participants), DMC provided an inferior account of the data than DSTP and SSP when assessing the full diffusion variants. For every participant in White et al. (2011) and Servant et al. (2015), and 12/16 participants in (Ulrich et al., 2015), there was at least strong evidence for DMC providing the poorest account of the data, and for every participant in every study there was at least strong evidence for DMC not being the best model of the data. These findings demonstrate that DMC provides a substantially poorer account of the flanker task than DSTP and SSP. Consequently, SSP and DSTP should be favored when drawing inferences (e.g., measures of cognitive control) from flanker data.

Our study also investigated why DMC provided a poorer explanation of the flanker task than DSTP and DMC. Specifically, we assessed DCDF, CAF, ELI, and DF plots, which unveiled several issues: an under-estimation of the accuracy in later incompatible 
trials responses, an under-estimation of the response time of errors relative to correct responses, and an over-estimation of the difference between incompatible and compatible trial correct response time in later trials. These misfits all appear to point to one common issue: a continuing influence of DMC's automatic process in later stages of the decision. This issue was confirmed by an analysis of the expected mean of the automatic activation $\left(E\left[X_{a}(t)\right]\right)$ computed from parameters that best accounted for the entire response time distributions. Under this parameterisation, $E\left[X_{a}(t)\right]$ either remained stable or continued to increase over decision time, resulting in a continued or increasing influence of the flankers over time, and an inability to capture many aspects of the data. Note that this does not indicate a failure of DMC to capture these trends under all possible parameterisations, but rather, a failure of DMC to capture these trends under the parameter values that provide the best account of the complete data.

Given the apparent failure of the DMC, it might be tempting to conclude that the visual attention explanations of the flanker task appear to be better than the automaticity explanation. However, prominent theories of automaticity, such as Logan's instance theory (Logan, 1988), assert that automaticity is a consequence of attention, suggesting that these models have underlying theoretical similarities. The observed failure of the DMC might be rooted in the general assumption of summing independent automatic and controlled activations at the decision stage, or to ancillary assumptions such as the choice of a gamma function to model the automatic activation.

It should also be noted that our study focused solely on the flanker task, and the inferiority of DMC in the flanker task should not be extrapolated to a general failure of DMC in all conflict tasks. We chose to compare these models on the flanker task as it is the task that all three models were designed to account for, and therefore, provides the fairest comparison. When considering other conflict tasks, such as the Simon task, DMC 
has previously been shown to be able to provide an accurate account of the qualitative trends in these tasks, such as the delta functions (Ulrich et al., 2015). However, these previous findings should also be taken with a grain of salt based on the findings of our study. Specifically, DMC has also been shown to be able to provide an accurate account of these qualitative trends in the flanker task, though as shown in our study, this is not the case when DMC is fit to the entire empirical data. Therefore, we believe that future research should investigate the ability of DMC to account for qualitative trends in conflict tasks while constrained by the entire data. Note that we are in no way attempting to claim that DMC fails to provide an accurate account of the qualitative trends in other conflict tasks, such as the Simon task, and we believe that such a conclusion would be unjustified based on the findings of our study. Furthermore, we again wish to emphasize that the results of our study are only applicable to understanding the process that underlies the flanker task, and not conflict tasks in general. However, we believe that investigating other conflict tasks - or several conflict tasks jointly - with our proposed methodology is an important avenue for future research, which would greatly aid our understanding of how conflict processing generally operates. Moreover, we believe that connecting cognitive models with neurophysiological data is fundamental to understanding how cognitive processes are implemented in the brain, and that future research should strive to better incorporate theories about these connections within the frameworks of the conflict diffusion models, and to compare these theories by constraining the models to account for both behavioural and neurophysiological data (e.g., Purcell et al., 2010; Purcell, Schall, Logan, \& Palmeri, 2012; Servant et al., 2015, 2016; Servant, Tillman, Schall, Logan, \& Palmeri, 2019).

When comparing DSTP and SSP, the two models based on visual attention research, the results were mixed. The White et al. (2011) data appeared to provide clear evidence in favour of SSP, with 17/25 participants showing at least strong evidence in favour of SSP and 
only 2/25 participants showing strong evidence in favour of DSTP. Further investigation revealed that DSTP's poorer performance in these data appeared to be the result of an under-prediction of the response time of errors relative to correct responses in incompatible trials. However, both the Ulrich et al. (2015) and Servant et al. (2015) data sets showed no clear winner. In Ulrich et al. (2015), 3/16 participants each showed at least strong evidence in favour of each model, with several more showing weaker evidence in favour of DSTP, suggesting that DSTP provided a slightly better account of these data. The Servant et al. (2015) data set provided a near-perfect split between the models, with 6/12 participants each favouring DSTP and SSP, though only 5/6 favouring SSP showing at least strong evidence. Although it may appear that SSP generally provides a superior explanation to DSTP based on the strong evidence in favour of SSP in the data of White et al. (2011), this superiority might also be explained by methodological discrepancies between studies. Specifically, one potentially key difference is the response-stimulus interval (RSI), which was 350ms in the White et al. (2011) data, but much longer in the Servant et al. (1,000ms) and Ulrich et al. (1,500ms) data sets. Hübner and Töbel (2012) argued that the shrinking spotlight component of the SSP predicts an improvement of attentional selectivity that is generally too fast to capture the corresponding improvement of accuracy in the incompatible condition. Importantly, they found that a reduction of RSI (up to $350 \mathrm{~ms}$, as in the White et al. (2011) data set) increases spatial selectivity, resulting in an overall reduction in the error rate. Therefore, our observed superiority of the SSP over the DSTP under the conditions of White et al. (2011) may not be particularly surprising, given that the short RSI can eliminate a key shortcoming of SSP, and that SSP is generally more parsimonious than DSTP. It should also be noted that for our model recovery assessment based on the estimated parameters (see the Supplementary Materials), DSTP was difficult to recover when it was the true generating model, with SSP closely mimicking the data 
generated by DSTP (see Wagenmakers, Ratcliff, Gomez, \& Iverson, 2004 for a discussion of model mimicry). This is in contrast to the findings of (White et al., 2018), and suggests that under some parameterisations - such as those that best account for the data sets in our study - SSP can strongly mimic DSTP, which may make the superiority of SSP in the White et al. (2011) data set questionable.

One interesting question arising from our study is why the results were so mixed between DSTP and SSP for the Ulrich et al. (2015) and Servant et al. (2015) data sets. Although the cross-fitting assessment of White et al. (2018) found that these models are quantitatively distinguishable over a wide spectrum of parameter combinations, the models still may be quantitatively similar in some circumstances, where the superior model is decided by minor trends in the response time distributions that may not be consistent across people. The main theoretical divergence between the models concerns whether attention selectivity improves in a discrete (DSTP) or continuous (SSP) manner, resulting in a discrete or continuous change in decision evidence. However, the latency at which selection occurs within DSTP is variable from trial to trial - based on the target identification process - which could be considered to globally approximate a continuous selection process. In addition, the shrinking spotlight mechanism of the SSP can approximate a discrete selection process if the shrinking rate $S D_{r}$ is large compared to the initial spotlight width $S D_{0}$. We found this to be the case, particularly in the Ulrich et al. (2015) and Servant et al. (2015) data sets where the results were mixed. Estimated parameters (posterior means averaged across subjects; see the Supplementary Material) showed $S D_{0}=8.08$, 2.98, 4.67 and $S D_{r}=0.17 / \mathrm{ms}, 0.19 / \mathrm{ms}, 0.13 / \mathrm{ms}$ for the White et al. (2011), the Ulrich et al. (2015), and the Servant et al. (2015) data sets respectively. Consequently, the latency at which attention is fully focused on the target (i.e., $S D(t)=.001$ ) relative to decision onset is fast $(48 \mathrm{~ms}, 23 \mathrm{~ms}, 24 \mathrm{~ms})$, which approximates an abrupt change in decision evidence. 
This also provides a potential explanation for why SSP was able to strongly mimic DSTP in our model recovery assessment, but that White et al. (2018) found clear separation between these models. Future research may benefit from detailed assessments of selection dynamics within SSP and DSTP, and how similar they can become in different regions of the parameter space.

Our findings also appeared to show a general superiority for the "full" diffusion variants of the models - which include between-trial variability parameters for drift rate, starting point, and non-decision time - over the "simple" diffusion variants - which include no between-trial variability parameters. For every participant in the White et al. (2011) and Servant et al. (2015) experiments, the full diffusion variant of each model produced a higher marginal likelihood than its simple diffusion counterpart, and this was also the case for the vast majority of cases in Ulrich et al. (2015). These findings suggest that these between-trial variability parameters are important for explaining certain data trends in conflict tasks, which is perhaps not surprising given their importance in explaining benchmark response time phenomena in standard decision-making tasks (Ratcliff, 1978; Ratcliff \& Rouder, 1998; Ratcliff \& Tuerlinckx, 2002). Importantly, previous applications and comparisons of these models have only used the "simple" variants, which appears to be a major caveat, and future studies should aim to apply these models within the full diffusion framework.

\section{Methodological impacts of our study}

Our study made key advancements in the methodology for determining which model provides the best explanation of an unknown psychological process, making the comparisons through Bayes factors, which have only been implemented in a few instances for cognitive models of choice response time (Evans \& Brown, 2018; Annis et al., 2019; Evans \& Annis, 2019; Evans, Bennett, \& Brown, 2018) due to recent advancements in methods of marginal 
likelihood approximation (Annis et al., 2019; Evans \& Annis, 2019). Most notably, the models that we assessed have computationally intractable likelihood functions, meaning that the TIDE marginal likelihood approximation method had to be integrated with PDA, to obtain a pseudo-likelihood for the models through simulation. This combination of PDA and TIDE - which we term pseudo-likelihood Bayes factors - has potential broad-reaching implications, as any model that can be simulated can also, in theory, have a marginal likelihood calculated for it, meaning that the comparison of any models can be done via Bayes factors. We believe that using these methods together provides a crucial step forward in how researchers compare computational models with unknown or intractable likelihood functions, such as the comparison between conflict models of the flanker task that we performed within our study.

Our study also offered insight into why DSTP and SSP provide a better explanation of the psychological process underlying the flanker task than DMC. Importantly, our study makes a clear distinction between determining which model provides the best explanation of the underlying process - a question best answered by principled model selection methods - and understanding why some models outperformed others - a question best answered by visual assessments of qualitative trends in the data (see Evans, 2019c for a more detailed discussion). Research comparing cognitive models often focuses solely on the question of which or why, either only performing model selection to determine which model is best, which leaves understanding why this is the case to future research, or skipping model selection and moving straight to visual assessments of qualitative trends, which ignores large portions of the empirical data (Evans, 2019c) and provides no correction for model flexibility (Roberts \& Pashler, 2000). Our study provides a clear example for how both questions can be answered within a single study. Firstly, researchers should use principled model selection techniques to determine which model provides the best account of an 
underlying process, and then use visual assessments of qualitative trends to understand why this is the case. We believe that our combination of answering the which and why has provided valued insight into the flanker task, and that future studies could benefit from similar assessments.

Furthermore, our study used a series of robustness analyses to ensure the consistency of our inferences across different ways that the models could potentially have been applied (see the Supplementary Materials). Specifically, we assessed the robustness of our inferences against simple simulation requirements, general specification of the functional form of DMC, and the prior distributions used to calculate Bayes factors. One robustness analysis that we believe is of general relevance to future comparisons between cognitive models is our empirical Bayes robustness analysis, which we used to ensure the robustness of our inferences across different prior distributions. This analysis took the estimated posterior distributions for the parameters that are unique to $\operatorname{DMC}(\tau, \zeta$, and $\alpha)$, and re-calculated the Bayes factors using these posterior distributions as the prior distributions, meaning that DMC essentially had the perfect prior distributions for its unique parameters, providing it with a unfair advantage over the other models. However, even with this extra advantage, DMC was still generally inferior to DSTP and SSP, meaning that our inferences were robust against the prior distributions specified for the parameters that are unique to DMC. One important thing to consider is how exactly the empirical Bayes robustness analysis should be interpreted. In our view, a reversal in inferences in the empirical Bayes robustness assessment does not mean that the previous inferences were incorrect, as there are known issues with empirical Bayes approaches, and more generally empirical Bayes will be biased towards more flexible models. However, seeing no change in inferences provides a strong assurance that even the perfect choice of priors for these data would not have resulted in a change in inferences, and seeing a reversal in inferences suggests that the prior 
distributions of the models, and their influence on the inferences made, should be more carefully explored. It should also be noted that our empirical Bayes robustness analysis is not useful when comparing nested models - where one model in the comparison is a subset of the other model - as the empirical Bayes approach will be strongly biased towards the more complex model (i.e., a conceptually similar issue to the one discussed by Gronau \& Wagenmakers, 2019 with Bayesian leave-one-out cross-validation).

Although we believe that our proposed pseudo-likelihood Bayes factors provide a crucial step forward in how researchers compare computational models with unknown or intractable likelihood functions, there are also limitations of our methodology that should be acknowledged. Specifically, our methodology relies on several layers of approximation: PDA to approximate the likelihood function, MCMC to approximate the posterior distributions, and TI to approximate the marginal likelihood. Importantly, large approximation error in any of these layers, or the propagation of error across several of these layers, could result in unreliable inferences that are the result of noise in the approximation process. Approximation errors in lower levels of the process, such as the pseudo-likelihood created via simulation, will also filter through into the higher levels of the process, such as the posterior estimation via MCMC, meaning small approximation errors in the pseudo-likelihood could result in larger approximation errors in the MCMC process. Furthermore, these approximation errors are likely to become more prevalent in sloppy, high-dimensional models (i.e., models with $20+$ highly correlated parameters), as the likelihood surface (i.e., the probability of the data given the parameters, over the complete range of different parameter values) becomes increasingly flat, and therefore, the movement through the parameter space becomes increasingly dominated by the approximation error. We attempted to mitigate the impact of any potential approximation errors within our study by re-running the entire process 5 times, obtaining 5 approximations to the marginal likelihood for each 
comparison, and making inferences using the end-points of these ranges. Therefore, the strength of evidence we reported was based on the lowest obtained marginal likelihood for the superior model compared to the highest obtained marginal likelihood for the inferior model, which we believe provides a method of comparison that is robust to potential approximation errors. However, more generally, we believe that approximation should be avoided where possible. For example, in simple statistical models where the marginal likelihood can be calculated analytically, marginal likelihood approximation methods such as TI should not be used in place of these analytic methods. Although this is unlikely to be possible in all cognitive models, many cognitive models may have likelihood functions that can be obtained analytically or numerically, which would avoid the need for the PDA level of approximation. Therefore, in cases where the likelihood function can be obtained analytically or numerically, and is computationally feasible to calculate, then we believe that these deterministic likelihood functions should be used over simulation-based pseudolikelihoods, removing a level of approximation, and meaning that inferences are more likely to be reliable.

It should also be noted that there have been several other recently proposed methods of simulation-based Bayesian inference. Importantly, these methods may provide interesting alternatives to our pseudo-likelihood Bayes factor methodology, especially when researchers may have questions that may not be answerable through Bayes factors. Specifically, our pseudo-likelihood Bayes factor methodology is only designed for hypothesis testing: that is, comparing two or more models to determine which model provides the best explanation of a psychological process. However, researchers are often interested in other goals when applying cognitive models, such as estimating the latent parameters of the psychological process. In these situations our pseudo-likelihood Bayes factor methodology will not be of much value to researchers, but several other simulation-based methods exist 
to provide efficient parameter estimation. For example, recent advancements have combined $\mathrm{ABC}$ methods with neural networks, which attempt to provide efficient methods of estimating the posterior distribution by learning the summary statistics of simulated data from parameter values across the range of the parameter space, and using this to efficiently sample from the posterior distribution (Lueckmann et al., 2018) or directly estimate the early moments of the posterior distribution (Radev et al., 2019). These methods provide principled and efficient techniques for answering different research questions - focused on parameter estimation - for simulation-based models, and form an important complement to our pseudo-likelihood Bayes factor methodology, which focuses on hypothesis testing. 


\section{References}

Ambrosi, S., Servant, M., Blaye, A., \& Burle, B. (2019). Conflict processing in kindergarten children: New evidence from distribution analyses reveals the dynamics of incorrect response activation and suppression. Journal of experimental child psychology, 177, 36-52.

Annis, J., Evans, N. J., Miller, B. J., \& Palmeri, T. J. (2019). Thermodynamic integration and steppingstone sampling methods for estimating bayes factors: A tutorial. Journal of mathematical psychology, 89, 67-86.

Beaumont, M. A., Zhang, W., \& Balding, D. J. (2002). Approximate bayesian computation in population genetics. Genetics, 162(4), 2025-2035.

Brown, S. D., Ratcliff, R., \& Smith, P. L. (2006). Evaluating methods for approximating stochastic differential equations. Journal of Mathematical Psychology, 50, 402-410.

Browne, M. (2000). Cross-validation methods. Journal of Mathematical Psychology, 44, 108ñ-132.

Carlin, B. P., \& Chib, S. (1995). Bayesian model choice via Markov chain Monte Carlo methods. Journal of the Royal Statistical Society, Series B, 57, 473-484.

Cohen, J. D., Servan-Schreiber, D., \& McClelland, J. L. (1992). A parallel distributed processing approach to automaticity. The American journal of psychology, 239-269.

Cornuet, J.-M., Santos, F., Beaumont, M. A., Robert, C. P., Marin, J.-M., Balding, D. J., ... Estoup, A. (2008). Inferring population history with diy abc: a user-friendly approach to approximate bayesian computation. Bioinformatics, $24(23), 2713-2719$.

Csilléry, K., Blum, M. G., Gaggiotti, O. E., \& François, O. (2010). Approximate bayesian computation (abc) in practice. Trends in ecology $\&$ evolution, 25(7), 410-418.

De Jong, R., Liang, C.-C., \& Lauber, E. (1994). Conditional and unconditional automaticity: A dual-process model of effects of spatial stimulus-response correspondence. Journal of Experimental Psychology: Human Perception and Performance, 20, 731-750.

Eriksen, B. A., \& Eriksen, C. W. (1974). Effects of noise letters upon the identification of a target letter in a nonsearch task. Perception 83 Psychophysics, 16, 143-149.

Eriksen, C. W., \& James, J. D. S. (1986). Visual attention within and around the field of focal attention: A zoom lens model. Perception \& psychophysics, 40(4), 225-240. 
Estes, W. K. (1956). The problem of inference from curves based on group data. Psychological Bulletin, 53, 134-140.

Evans, N. J. (2019a). Assessing the practical differences between model selection methods in inferences about choice response time tasks. Psychonomic bulletin $\mathcal{E}$ review, 1-29.

Evans, N. J. (2019b). A method, framework, and tutorial for efficiently simulating models of decision-making. Behavior Research Methods, 1-15.

Evans, N. J. (2019c). What factors are most important in finding the best model of a psychological process? Comment on Navarro (2018). PsyArXiv preprint.

Evans, N. J., \& Annis, J. (2019). Thermodynamic integration via differential evolution: A method for estimating marginal likelihoods. Behavior research methods, 1-18.

Evans, N. J., Bennett, A. J., \& Brown, S. D. (2018). Optimal or not; depends on the task. Psychonomic bulletin \& review, 1-8.

Evans, N. J., \& Brown, S. D. (2017). People adopt optimal policies in simple decision-making, after practice and guidance. Psychonomic Bulletin \&3 Review, 24(2), 597-606.

Evans, N. J., \& Brown, S. D. (2018). Bayes factors for the linear ballistic accumulator model of decision-making. Behavior research methods, 50(2), 589-603.

Evans, N. J., Brown, S. D., Mewhort, D. J., \& Heathcote, A. (2018). Refining the law of practice. Psychological review, 125(4), 592.

Evans, N. J., Hawkins, G., \& Brown, S. (2019). The role of passing time in decision-making. Journal of Experimental Psychology: Learning, Memory, \& Cognition.

Evans, N. J., \& Hawkins, G. E. (2019). When humans behave like monkeys: Feedback delays and extensive practice increase the efficiency of speeded decisions. Cognition, 184, 11-18.

Evans, N. J., Hawkins, G. E., Boehm, U., Wagenmakers, E.-J., \& Brown, S. D. (2017). The computations that support simple decision-making: A comparison between the diffusion and urgency-gating models. Scientific Reports, 7, 16433.

Evans, N. J., Holmes, W. R., \& Trueblood, J. S. (2019). Response-time data provide critical constraints on dynamic models of multi-alternative, multi-attribute choice. Psychonomic Bulletin Review.

Evans, N. J., Howard, Z. L., Heathcote, A., \& Brown, S. D. (2017). Model flexibility analysis does 
not measure the persuasiveness of a fit. Psychological review, $124(3), 339$.

Evans, N. J., Rae, B., Bushmakin, M., Rubin, M., \& Brown, S. D. (2017). Need for closure is associated with urgency in perceptual decision-making. Memory $\&$ Cognition, 1-13.

Evans, N. J., Steyvers, M., \& Brown, S. D. (2018). Modeling the covariance structure of complex datasets using cognitive models: An application to individual differences and the heritability of cognitive ability. Cognitive science, 42(6), 1925-1944.

Friel, N., \& Pettitt, A. N. (2008). Marginal likelihood estimation via power posteriors. Journal of the Royal Statistical Society: Series B (Statistical Methodology), 70(3), 589-607.

Gratton, G., Coles, M. G. H., Sirevaag, E. J., Eriksen, C. W., \& Donchin, E. (1988). Pre- and poststimulus activation of response channels: A psychophysiological analysis. Journal of Experimental Psychology: Human Perception and Performance, 14, 331-344.

Green, P. J. (1995). Reversible jump Markov chain Monte Carlo computation and Bayesian model determination. Biometrika, 82, 711-732.

Grelaud, A., Robert, C. P., Marin, J.-M., Rodolphe, F., Taly, J.-F., et al. (2009). Abc likelihood-free methods for model choice in gibbs random fields. Bayesian Analysis, 4(2), 317-335.

Gronau, Q. F., Sarafoglou, A., Matzke, D., Ly, A., Boehm, U., Marsman, M., ... Steingroever, H. (2017). A tutorial on bridge sampling. Journal of mathematical psychology, 81, 80-97.

Gronau, Q. F., \& Wagenmakers, E.-J. (2019). Limitations of bayesian leave-one-out cross-validation for model selection. Computational Brain Behavior, 2(1), 1-11.

Hawkins, G. E., Forstmann, B. U., Wagenmakers, E.-J., Ratcliff, R., \& Brown, S. D. (2015). Revisiting the evidence for collapsing boundaries and urgency signals in perceptual decisionmaking. Journal of Neuroscience, 35, 2476-2484.

Heathcote, A., Brown, S., \& Mewhort, D. J. K. (2000). The power law repealed: The case for an exponential law of practice. Psychonomic Bulletin \& Review, 7, 185-207.

Holmes, W. R. (2015). A practical guide to the probability density approximation (pda) with improved implementation and error characterization. Journal of Mathematical Psychology, $68,13-24$.

Hübner, R., Steinhauser, M., \& Lehle, C. (2010). A dual-stage two-phase model of selective attention. Psychological review, 117(3), 759. 
Hübner, R., \& Töbel, L. (2012). Does attentional selectivity in the flanker task improve discretely or gradually? Frontiers in psychology, 3, 434.

Hübner, R., \& Töbel, L. (2019). Conflict resolution in the eriksen flanker task: Similarities and differences to the simon task. PLoS One, 14 (3), e0214203.

Jeffreys, H. (1961). Theory of probability. Oxford, UK: Oxford University Press.

Kahneman, D., \& Tversky, A. (1979). Prospect theory: An analysis of decision under risk. Econometrica, 47, 263-291.

Kass, R. E., \& Raftery, A. E. (1995). Bayes factors. Journal of American Statistical Association, 90(430), 773-795.

Laming, D. R. J. (1968). Information theory of choice-reaction times. London: Academic Press.

Lartillot, N., \& Philippe, H. (2006). Computing bayes factors using thermodynamic integration. Systematic biology, 55(2), 195-207.

Logan, G. D. (1980). Attention and automaticity in stroop and priming tasks: Theory and data. Cognitive psychology, 12(4), 523-553.

Logan, G. D. (1988). Toward an instance theory of automatization. Psychological Review, 95, $492-527$.

Logan, G. D. (1996). The code theory of visual attention: an integration of space-based and object-based attention. Psychological review, 103(4), 603.

Lueckmann, J.-M., Bassetto, G., Karaletsos, T., \& Macke, J. H. (2018). Likelihood-free inference with emulator networks. arXiv preprint arXiv:1805.09294.

Mahani, M.-A. N., Bausenhart, K. M., Ahmadabadi, M. N., \& Ulrich, R. (2018). Multimodal simon effect: A multimodal extension of the diffusion model for conflict tasks. Frontiers in human neuroscience, 12, 507.

Myung, I. J. (2000). The importance of complexity in model selection. Journal of Mathematical Psychology, 44, 190-204.

Myung, I. J., \& Pitt, M. A. (1997). Applying Occam's razor in modeling cognition: A Bayesian approach. Psychonomic Bulletin \& Review, 4, 79-95.

Pritchard, J. K., Seielstad, M. T., Perez-Lezaun, A., \& Feldman, M. W. (1999). Population growth of human y chromosomes: a study of y chromosome microsatellites. Molecular biology and 
evolution, 16(12), 1791-1798.

Purcell, B. A., Heitz, R. P., Cohen, J. Y., Schall, J. D., Logan, G. D., \& Palmeri, T. J. (2010). Neurally constrained modeling of perceptual decision making. Psychological Review, 117, $1113-1143$.

Purcell, B. A., Schall, J. D., Logan, G. D., \& Palmeri, T. J. (2012). From salience to saccades: Multiple-alternative gated stochastic accumulator model of visual search. Journal of Neuroscience, 32, 3433-3446.

Radev, S. T., Mertens, U. K., Voss, A., \& Köthe, U. (2019). Towards endtoend likelihoodfree inference with convolutional neural networks. British Journal of Mathematical and Statistical Psychology.

Ratcliff, R. (1978). A theory of memory retrieval. Psychological Review, 85, 59-108.

Ratcliff, R. (1980). A note on modeling accumulation of information when the rate of accumulation changes over time. Journal of Mathematical Psychology.

Ratcliff, R., \& Rouder, J. N. (1998). Modeling response times for two-choice decisions. Psychological Science, $9(5), 347-356$.

Ratcliff, R., Smith, P. L., Brown, S. D., \& McKoon, G. (2016). Diffusion decision model: Current issues and history. Trends in Cognitive Sciences, 20, 260-281.

Ratcliff, R., \& Tuerlinckx, F. (2002). Estimating parameters of the diffusion model: Approaches to dealing with contaminant reaction times and parameter variability. Psychonomic Bulletin \& Review, 9, 438-481.

Rigoux, L., Stephan, K. E., Friston, K. J., \& Daunizeau, J. (2014). Bayesian model selection for group studiesrevisited. Neuroimage, 84, 971-985.

Robert, C. P., Cornuet, J.-M., Marin, J.-M., \& Pillai, N. S. (2011). Lack of confidence in approximate bayesian computation model choice. Proceedings of the National Academy of Sciences, $108(37), 15112-15117$.

Roberts, S., \& Pashler, H. (2000). How persuasive is a good fit? A comment on theory testing in psychology. Psychological Review, 107, 358-367.

Roitman, J. D., \& Shadlen, M. N. (2002). Responses of neurons in the lateral intraparietal area during a combined visual discrimination reaction time task. Journal of Neuroscience, 22, 
9475-9489.

Schwarz, G. (1978). Estimating the dimension of a model. Annals of Statistics, 6, 461-464.

Schwarz, W., \& Miller, J. (2012). Response time models of delta plots with negative-going slopes. Psychonomic Bulletin \& Review, 19(4), 555-574.

Servant, M., Gajdos, T., \& Davranche, K. (2018). ELF: A new measure of response capture. Psychonomic Bulletin Review, 1-9.

Servant, M., Montagnini, A., \& Burle, B. (2014). Conflict tasks and the diffusion framework: Insight in model constraints based on psychological laws. Cognitive psychology, 72, 162-195.

Servant, M., Tillman, G., Schall, J. D., Logan, G. D., \& Palmeri, T. J. (2019). Neurally constrained modeling of speed-accuracy tradeoff during visual search: gated accumulation of modulated evidence. Journal of neurophysiology, 121(4), 1300-1314.

Servant, M., van Wouwe, N., Wylie, S. A., \& Logan, G. D. (2018). A model-based quantification of action control deficits in parkinson's disease. Neuropsychologia, 111, 26-35.

Servant, M., White, C., Montagnini, A., \& Burle, B. (2015). Using covert response activation to test latent assumptions of formal decision-making models in humans. Journal of Neuroscience, $35(28), 10371-10385$.

Servant, M., White, C., Montagnini, A., \& Burle, B. (2016). Linking theoretical decision-making mechanisms in the simon task with electrophysiological data: A model-based neuroscience study in humans. Journal of Cognitive Neuroscience, 28(10), 1501-1521.

Shiffrin, R. M., Lee, M. D., Kim, W., \& Wagenmakers, E.-J. (2008). A survey of model evaluation approaches with a tutorial on hierarchical Bayesian methods. Cognitive Science, 32, 12481284 .

Silverman, B. W. (1986). Density estimation for statistics and data analysis. London: Chapman \& Hall.

Simon, J. R. (1969). Reactions toward the source of stimulation. Journal of experimental psychology, $81(1), 174$.

Simon, J. R., \& Rudell, A. P. (1967). Auditory sr compatibility: the effect of an irrelevant cue on information processing. Journal of applied psychology, $51(3), 300$.

Smith, P. L. (2000). Stochastic dynamic models of response time and accuracy: A foundational 
primer. Journal of Mathematical Psychology, 44, 408-463.

Stephan, K. E., Penny, W. D., Daunizeau, J., Moran, R. J., \& Friston, K. J. (2009). Bayesian model selection for group studies. Neuroimage, 46 (4), 1004-1017.

Stone, M. (1960). Models for choice-reaction time. Psychometrika, 25, 251-260.

Stroop, J. R. (1935). Studies of interference in serial verbal reactions. Journal of experimental psychology, 18(6), 643 .

Tavare, S., Balding, D. J., Griffiths, R. C., \& Donnelly, P. (1997). Inferring coalescence times from DNA sequence data. Genetics, 145(2), 505-518.

Ter Braak, C. J. (2006). A markov chain monte carlo version of the genetic algorithm differential evolution: easy bayesian computing for real parameter spaces. Statistics and Computing, $16(3), 239-249$.

Turner, B. M., Dennis, S., \& Van Zandt, T. (2013). Likelihood-free bayesian analysis of memory models. Psychological review, 120(3), 667.

Turner, B. M., \& Sederberg, P. B. (2012). Approximate Bayesian computation with differential evolution. Journal of Mathematical Psychology, 56, 375-385.

Turner, B. M., \& Sederberg, P. B. (2014). A generalized, likelihood-free method for posterior estimation. Psychonomic bulletin \& review, 21(2), 227-250.

Turner, B. M., Sederberg, P. B., Brown, S. D., \& Steyvers, M. (2013). A method for efficiently sampling from distributions with correlated dimensions. Psychological Methods, 18, 368-384.

Turner, B. M., \& Van Zandt, T. (2012). A tutorial on approximate bayesian computation. Journal of Mathematical Psychology, 56(2), 69-85.

Turner, B. M., \& Van Zandt, T. (2014). Hierarchical approximate bayesian computation. Psychometrika, $79(2), 185-209$.

Tversky, A., \& Kahneman, D. (1974). Judgment under uncertainty: Heuristics and biases. Science, $185(4157), 1124-1131$.

Ulrich, R., Schröter, H., Leuthold, H., \& Birngruber, T. (2015). Automatic and controlled stimulus processing in conflict tasks: Superimposed diffusion processes and delta functions. Cognitive Psychology, 78, 148-174.

Wagenmakers, E.-J., Ratcliff, R., Gomez, P., \& Iverson, G. J. (2004). Assessing model mimicry 
using the parametric bootstrap. Journal of Mathematical Psychology, 48, 28-50.

White, C. N., Brown, S., \& Ratcliff, R. (2012). A test of bayesian observer models of processing in the eriksen flanker task. Journal of Experimental Psychology: Human Perception and Performance, 38(2), 489.

White, C. N., Ratcliff, R., \& Starns, J. J. (2011). Diffusion models of the flanker task: Discrete versus gradual attentional selection. Cognitive psychology, 63(4), 210-238.

White, C. N., Servant, M., \& Logan, G. D. (2018). Testing the validity of conflict drift-diffusion models for use in estimating cognitive processes: A parameter-recovery study. Psychonomic bulletin 83 review, 25(1), 286-301.

Yu, A. J., Dayan, P., \& Cohen, J. D. (2009). Dynamics of attentional selection: Towards a Bayesian account. Journal of Experimental Psychology: Human Perception and Performance, 35, 700717. 
Supplementary Materials for:

A comparison of conflict diffusion models in the flanker task through pseudo-likelihood Bayes factors 


\section{Comparisons with "simple" versions of models}

Here we display the log-marginal likelihoods for both the simple and complex variants of each model for the White et al. (2011; Figure 1, Tables 1-6), Ulrich et al. (2015; Figure 2, Tables 7-12), and Servant et al. (2015; Figure 3, Tables 13-18). The diamond points display the models in the "simple" framework, and the crosses display the models in the complex framework, with each of the 6 models on a different point of the x-axis. Different points of the same colour and shape, on the same point of the x-axis, display the 5 different approximated log-marginal likelihoods for the model. Specifically, blue displays DMC, red displays DSTP, and green displays SSP, with diamonds showing the simple versions and crosses showing the complex versions. The log-Bayes factors displayed are the same as those in the main text (i.e., between the full versions), as a point of reference.

For the White et al. (2011) and Servant et al. (2015) data sets, the "full" variants of the models perform at least as well as, and in most cases much better than, the "simple" variants, for all classes of models for all participants. For the Ulrich et al. (2015) data set there are a few exceptions to this rule, such as SSP for participant 10, DSTP for participant 7, and DMC for participant 16. Overall, this emphasises the importance of implementing the conflict diffusion models within the "full" diffusion framework when comparing their ability to account for empirical data, as this is greatly reduced when implemented as the simple variants. 

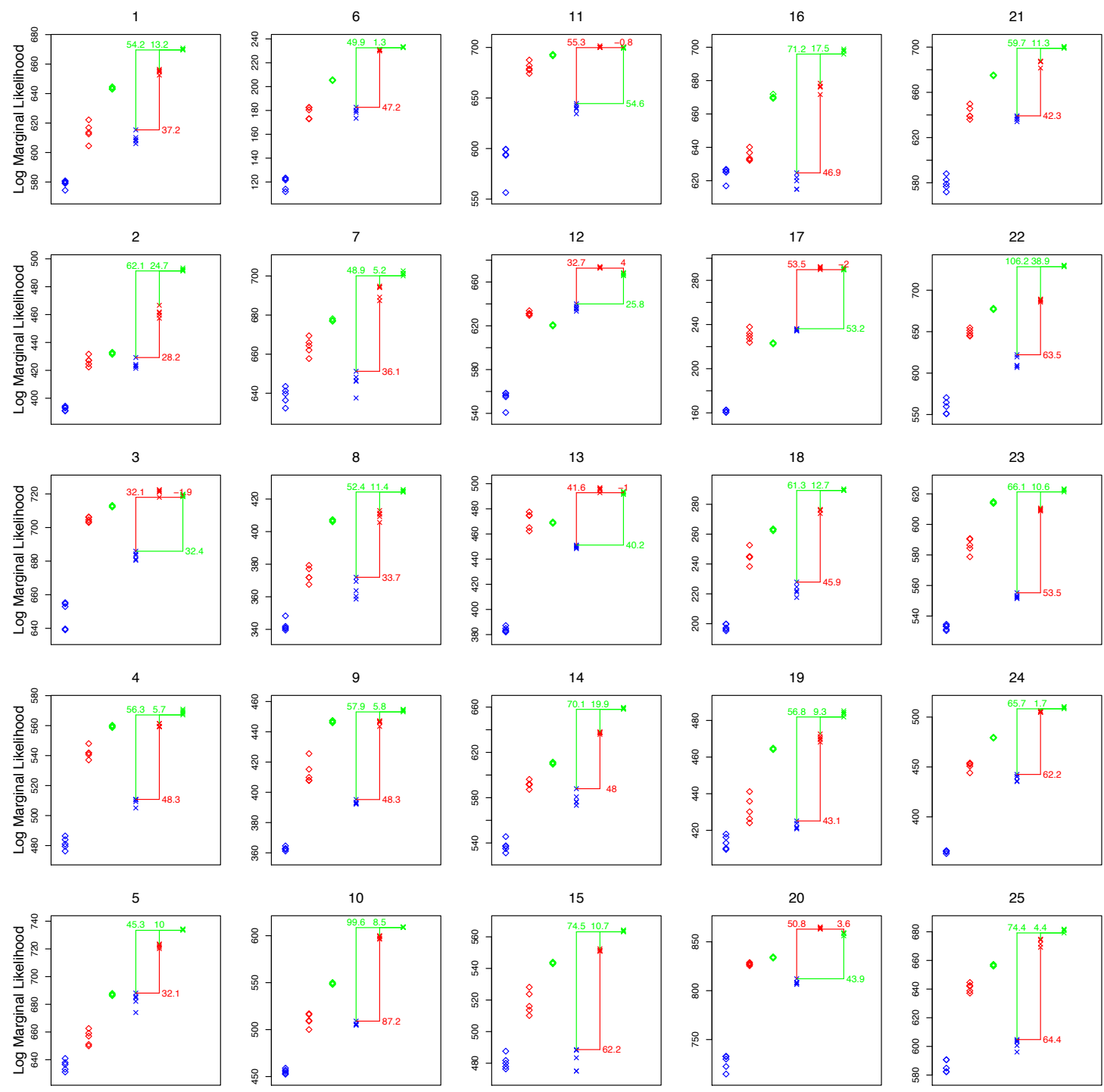

Figure 1. Bayes factor analysis for the White et al. (2011) data set. 
Table 1: The marginal likelihoods in the White et al. (2011) data set for the simple DMC, for all 5 estimation runs (columns) of all participants (rows).

\begin{tabular}{|c|c|c|c|c|c|}
\hline Subject & 1 & 2 & 3 & 4 & 5 \\
\hline 1 & 578.98 & 580.83 & 579.65 & 574.39 & 580.35 \\
\hline 2 & 394.36 & 392.97 & 393.53 & 391.28 & 390.84 \\
\hline 3 & 652.93 & 639.65 & 655.30 & 639.06 & 654.58 \\
\hline 4 & 480.96 & 479.50 & 486.42 & 476.29 & 483.94 \\
\hline 5 & 640.95 & 637.92 & 631.13 & 633.09 & 636.59 \\
\hline 6 & 111.85 & 122.66 & 121.65 & 123.43 & 114.02 \\
\hline 7 & 641.21 & 636.41 & 643.51 & 632.34 & 639.74 \\
\hline 8 & 340.43 & 341.07 & 339.52 & 348.28 & 341.87 \\
\hline 9 & 364.58 & 362.42 & 362.93 & 362.37 & 361.18 \\
\hline 10 & 455.44 & 456.87 & 458.95 & 453.60 & 452.45 \\
\hline 11 & 594.15 & 556.43 & 599.13 & 593.26 & 599.43 \\
\hline 12 & 558.58 & 558.24 & 540.79 & 555.12 & 556.39 \\
\hline 13 & 384.91 & 382.06 & 382.55 & 383.40 & 387.18 \\
\hline 14 & 545.64 & 537.56 & 536.88 & 535.00 & 531.19 \\
\hline 15 & 487.61 & 479.98 & 481.76 & 476.35 & 477.86 \\
\hline 16 & 626.85 & 625.15 & 625.16 & 616.88 & 626.26 \\
\hline 17 & 162.62 & 160.37 & 160.77 & 162.50 & 162.23 \\
\hline 18 & 199.80 & 197.19 & 195.22 & 196.24 & 199.73 \\
\hline 19 & 409.56 & 417.95 & 410.18 & 413.09 & 416.19 \\
\hline 20 & 733.01 & 732.38 & 714.71 & 730.01 & 722.44 \\
\hline 21 & 582.61 & 571.87 & 578.97 & 588.06 & 576.58 \\
\hline 22 & 551.15 & 570.47 & 559.63 & 550.85 & 564.59 \\
\hline 23 & 531.28 & 533.69 & 530.43 & 533.18 & 534.60 \\
\hline 24 & 365.99 & 366.02 & 365.96 & 363.19 & 364.96 \\
\hline 25 & 584.85 & 582.29 & 590.72 & 582.36 & 590.61 \\
\hline
\end{tabular}


Table 2: The marginal likelihoods in the White et al. (2011) data set for the complex DMC, for all 5 estimation runs (columns) of all participants (rows).

\begin{tabular}{|c|c|c|c|c|c|}
\hline Subject & 1 & 2 & 3 & 4 & 5 \\
\hline 1 & 608.77 & 607.79 & 615.37 & 606.05 & 610.17 \\
\hline 2 & 424.25 & 429.19 & 423.17 & 421.49 & 422.90 \\
\hline 3 & 681.15 & 680.45 & 684.29 & 683.46 & 685.89 \\
\hline 4 & 509.29 & 510.50 & 505.08 & 510.79 & 510.73 \\
\hline 5 & 682.14 & 684.71 & 688.09 & 685.70 & 674.06 \\
\hline 6 & 180.10 & 179.83 & 173.43 & 178.29 & 182.63 \\
\hline 7 & 646.50 & 651.22 & 637.57 & 646.10 & 648.15 \\
\hline 8 & 371.96 & 358.59 & 360.29 & 369.46 & 363.70 \\
\hline 9 & 392.12 & 392.82 & 392.73 & 393.41 & 395.22 \\
\hline 10 & 504.67 & 505.91 & 505.06 & 509.00 & 505.88 \\
\hline 11 & 640.25 & 642.57 & 634.40 & 644.49 & 639.30 \\
\hline 12 & 635.27 & 639.98 & 633.33 & 637.87 & 636.14 \\
\hline 13 & 450.29 & 448.37 & 450.13 & 449.09 & 451.19 \\
\hline 14 & 573.25 & 587.93 & 576.06 & 576.56 & 580.76 \\
\hline 15 & 475.06 & 483.44 & 488.62 & 488.17 & 475.05 \\
\hline 16 & 621.94 & 614.91 & 624.71 & 619.92 & 614.83 \\
\hline 17 & 234.38 & 235.14 & 236.18 & 234.14 & 235.99 \\
\hline 18 & 227.89 & 221.10 & 222.04 & 224.62 & 217.56 \\
\hline 19 & 420.80 & 421.60 & 423.84 & 421.32 & 425.12 \\
\hline 20 & 806.39 & 807.69 & 808.48 & 808.22 & 812.19 \\
\hline 21 & 638.09 & 634.08 & 636.20 & 639.21 & 636.24 \\
\hline 22 & 608.61 & 622.12 & 606.78 & 609.29 & 619.57 \\
\hline 23 & 551.94 & 552.74 & 551.45 & 555.23 & 553.39 \\
\hline 24 & 440.23 & 435.34 & 440.58 & 442.53 & 436.03 \\
\hline 25 & 603.91 & 603.15 & 596.07 & 604.78 & 600.67 \\
\hline
\end{tabular}


Table 3: The marginal likelihoods in the White et al. (2011) data set for the simple DSTP, for all 5 estimation runs (columns) of all participants (rows).

\begin{tabular}{|c|c|c|c|c|c|}
\hline Subject & 1 & 2 & 3 & 4 & 5 \\
\hline 1 & 612.59 & 622.18 & 616.87 & 613.62 & 604.50 \\
\hline 2 & 431.65 & 422.35 & 424.61 & 427.22 & 427.11 \\
\hline 3 & 702.94 & 706.33 & 704.58 & 703.54 & 706.06 \\
\hline 4 & 541.81 & 540.63 & 537.06 & 548.02 & 541.75 \\
\hline 5 & 649.96 & 657.01 & 662.58 & 659.13 & 651.08 \\
\hline 6 & 182.33 & 172.86 & 180.45 & 173.23 & 182.66 \\
\hline 7 & 665.93 & 669.41 & 657.73 & 664.34 & 662.11 \\
\hline 8 & 367.57 & 379.31 & 377.13 & 371.85 & 371.84 \\
\hline 9 & 425.57 & 407.68 & 409.86 & 408.02 & 415.22 \\
\hline 10 & 500.03 & 516.02 & 516.97 & 508.96 & 509.66 \\
\hline 11 & 678.87 & 674.23 & 682.09 & 687.57 & 677.71 \\
\hline 12 & 629.45 & 631.35 & 633.80 & 630.67 & 631.15 \\
\hline 13 & 474.52 & 462.33 & 477.57 & 465.08 & 474.95 \\
\hline 14 & 596.20 & 592.01 & 591.43 & 591.80 & 587.24 \\
\hline 15 & 516.05 & 528.14 & 513.64 & 523.75 & 510.14 \\
\hline 16 & 633.35 & 632.71 & 636.80 & 640.15 & 632.18 \\
\hline 17 & 227.13 & 223.78 & 229.79 & 232.16 & 237.81 \\
\hline 18 & 238.30 & 252.61 & 245.07 & 244.50 & 244.85 \\
\hline 19 & 441.15 & 430.14 & 435.85 & 424.05 & 426.32 \\
\hline 20 & 826.87 & 826.82 & 827.97 & 825.50 & 828.85 \\
\hline 21 & 645.67 & 649.77 & 636.03 & 639.08 & 639.02 \\
\hline 22 & 654.68 & 644.58 & 645.57 & 648.26 & 651.60 \\
\hline 23 & 590.34 & 586.45 & 584.42 & 590.77 & 578.77 \\
\hline 24 & 450.82 & 452.68 & 453.80 & 453.82 & 444.08 \\
\hline 25 & 642.57 & 638.80 & 637.19 & 644.62 & 641.94 \\
\hline
\end{tabular}


Table 4: The marginal likelihoods in the White et al. (2011) data set for the complex DSTP, for all 5 estimation runs (columns) of all participants (rows).

\begin{tabular}{|c|c|c|c|c|c|}
\hline Subject & 1 & 2 & 3 & 4 & 5 \\
\hline 1 & 652.52 & 655.09 & 654.57 & 655.81 & 656.41 \\
\hline 2 & 461.13 & 459.52 & 461.93 & 466.66 & 457.35 \\
\hline 3 & 722.46 & 721.59 & 717.98 & 721.08 & 722.39 \\
\hline 4 & 559.56 & 561.28 & 561.47 & 559.54 & 559.13 \\
\hline 5 & 720.19 & 723.47 & 721.38 & 722.43 & 723.41 \\
\hline 6 & 231.22 & 229.83 & 230.77 & 230.96 & 230.83 \\
\hline 7 & 687.36 & 694.84 & 694.26 & 689.18 & 694.03 \\
\hline 8 & 409.22 & 412.94 & 405.63 & 410.57 & 411.34 \\
\hline 9 & 447.05 & 447.30 & 443.56 & 446.16 & 446.60 \\
\hline 10 & 597.27 & 599.13 & 600.12 & 596.22 & 599.19 \\
\hline 11 & 701.64 & 700.08 & 700.01 & 700.22 & 699.83 \\
\hline 12 & 673.36 & 673.16 & 672.76 & 672.68 & 674.15 \\
\hline 13 & 495.40 & 495.98 & 492.81 & 495.10 & 496.82 \\
\hline 14 & 638.12 & 635.88 & 637.92 & 637.21 & 637.28 \\
\hline 15 & 551.55 & 550.87 & 552.45 & 550.91 & 551.10 \\
\hline 16 & 671.66 & 676.47 & 676.67 & 676.03 & 678.41 \\
\hline 17 & 292.48 & 292.06 & 291.43 & 289.68 & 290.79 \\
\hline 18 & 273.83 & 276.03 & 275.86 & 276.31 & 276.55 \\
\hline 19 & 471.17 & 469.51 & 472.63 & 468.18 & 470.11 \\
\hline 20 & 863.52 & 865.25 & 863.24 & 862.95 & 864.40 \\
\hline 21 & 687.25 & 687.15 & 687.64 & 687.07 & 681.54 \\
\hline 22 & 688.94 & 685.58 & 689.35 & 687.08 & 687.93 \\
\hline 23 & 609.85 & 609.90 & 609.23 & 610.73 & 608.73 \\
\hline 24 & 505.13 & 506.52 & 504.73 & 504.80 & 505.51 \\
\hline 25 & 674.79 & 674.24 & 674.82 & 669.19 & 671.43 \\
\hline
\end{tabular}


Table 5: The marginal likelihoods in the White et al. (2011) data set for the simple SSP, for all 5 estimation runs (columns) of all participants (rows).

\begin{tabular}{|c|c|c|c|c|c|}
\hline Subject & 1 & 2 & 3 & 4 & 5 \\
\hline 1 & 644.04 & 642.90 & 644.65 & 643.24 & 643.17 \\
\hline 2 & 431.39 & 433.01 & 432.27 & 432.79 & 432.21 \\
\hline 3 & 713.21 & 712.22 & 712.97 & 712.31 & 712.36 \\
\hline 4 & 558.63 & 559.89 & 560.30 & 559.30 & 559.29 \\
\hline 5 & 686.94 & 686.92 & 686.17 & 687.92 & 687.09 \\
\hline 6 & 205.43 & 205.81 & 205.55 & 205.26 & 204.99 \\
\hline 7 & 677.15 & 676.87 & 677.45 & 678.16 & 676.87 \\
\hline 8 & 407.24 & 407.43 & 405.93 & 406.34 & 406.84 \\
\hline 9 & 447.45 & 447.35 & 445.85 & 446.42 & 446.70 \\
\hline 10 & 548.09 & 550.24 & 548.21 & 549.26 & 549.13 \\
\hline 11 & 692.47 & 693.19 & 691.59 & 692.59 & 693.07 \\
\hline 12 & 619.91 & 619.91 & 620.85 & 620.88 & 620.97 \\
\hline 13 & 468.57 & 469.20 & 468.42 & 468.79 & 469.62 \\
\hline 14 & 611.49 & 609.45 & 610.99 & 609.85 & 610.50 \\
\hline 15 & 544.03 & 543.49 & 543.37 & 543.60 & 542.87 \\
\hline 16 & 670.23 & 671.91 & 669.91 & 669.33 & 669.40 \\
\hline 17 & 223.33 & 222.48 & 223.45 & 223.31 & 222.48 \\
\hline 18 & 263.26 & 263.09 & 263.07 & 262.17 & 263.57 \\
\hline 19 & 464.33 & 464.82 & 464.10 & 464.07 & 464.89 \\
\hline 20 & 833.79 & 834.25 & 833.22 & 834.03 & 834.65 \\
\hline 21 & 675.05 & 674.52 & 675.39 & 675.37 & 675.07 \\
\hline 22 & 678.35 & 676.91 & 677.18 & 677.29 & 676.45 \\
\hline 23 & 613.70 & 614.98 & 613.83 & 614.41 & 614.41 \\
\hline 24 & 479.81 & 478.94 & 478.90 & 479.66 & 479.21 \\
\hline 25 & 656.07 & 657.17 & 656.01 & 657.25 & 656.41 \\
\hline
\end{tabular}


Table 6: The marginal likelihoods in the White et al. (2011) data set for the complex SSP, for all 5 estimation runs (columns) of all participants (rows).

\begin{tabular}{|c|c|c|c|c|c|}
\hline Subject & 1 & 2 & 3 & 4 & 5 \\
\hline 1 & 669.63 & 669.83 & 669.90 & 670.84 & 669.59 \\
\hline 2 & 491.33 & 492.26 & 493.40 & 491.32 & 492.25 \\
\hline 3 & 719.86 & 718.29 & 719.63 & 719.09 & 718.67 \\
\hline 4 & 569.02 & 567.13 & 568.04 & 570.01 & 570.95 \\
\hline 5 & 733.72 & 733.42 & 733.99 & 734.24 & 734.41 \\
\hline 6 & 232.56 & 233.28 & 233.32 & 233.47 & 233.26 \\
\hline 7 & 701.42 & 701.24 & 700.99 & 700.08 & 702.64 \\
\hline 8 & 424.44 & 424.42 & 424.32 & 425.22 & 425.84 \\
\hline 9 & 453.85 & 454.89 & 454.28 & 453.13 & 454.59 \\
\hline 10 & 609.57 & 608.79 & 609.25 & 608.61 & 609.07 \\
\hline 11 & 700.60 & 699.39 & 699.83 & 699.50 & 699.13 \\
\hline 12 & 668.64 & 667.75 & 666.51 & 665.75 & 667.88 \\
\hline 13 & 493.36 & 493.81 & 493.35 & 491.43 & 492.26 \\
\hline 14 & 658.61 & 659.78 & 659.23 & 658.03 & 658.81 \\
\hline 15 & 564.38 & 563.59 & 564.14 & 563.28 & 563.17 \\
\hline 16 & 695.92 & 698.90 & 697.77 & 697.74 & 697.94 \\
\hline 17 & 289.81 & 290.33 & 290.15 & 291.68 & 289.37 \\
\hline 18 & 290.35 & 289.24 & 289.54 & 289.43 & 289.36 \\
\hline 19 & 484.23 & 485.25 & 483.35 & 481.90 & 484.29 \\
\hline 20 & 856.08 & 858.65 & 858.31 & 859.39 & 858.41 \\
\hline 21 & 699.95 & 700.25 & 698.93 & 699.41 & 700.64 \\
\hline 22 & 728.28 & 728.89 & 730.13 & 730.19 & 728.97 \\
\hline 23 & 621.28 & 622.22 & 623.13 & 623.17 & 622.99 \\
\hline 24 & 508.27 & 510.01 & 510.41 & 509.36 & 510.31 \\
\hline 25 & 679.19 & 681.82 & 681.18 & 680.80 & 681.78 \\
\hline
\end{tabular}



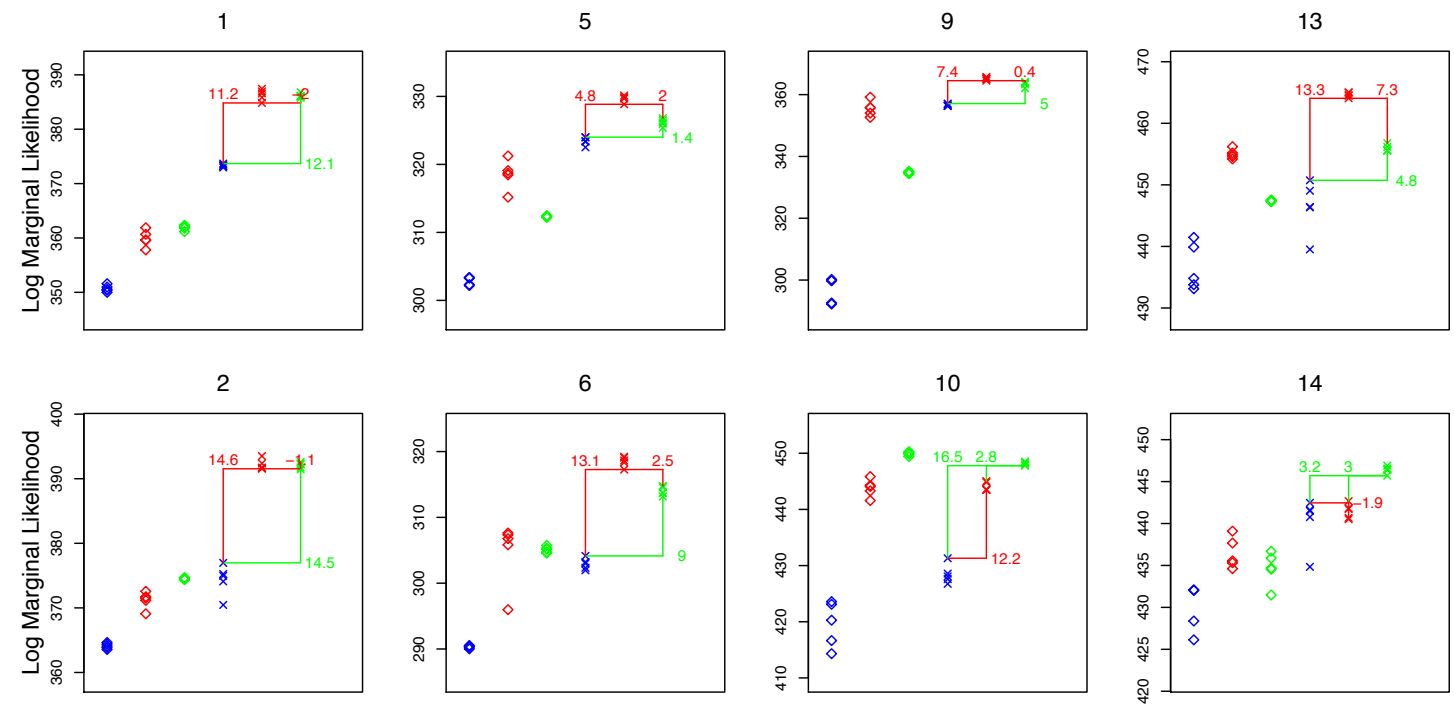

6

10
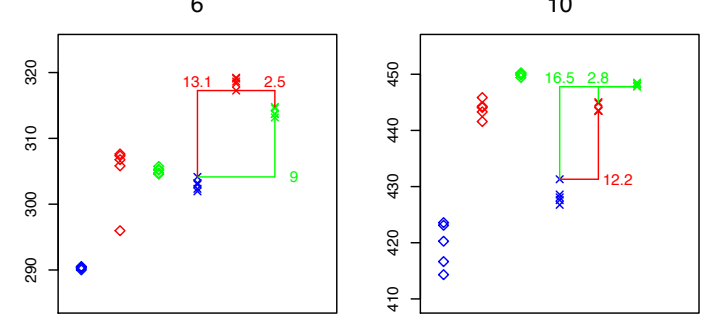

14
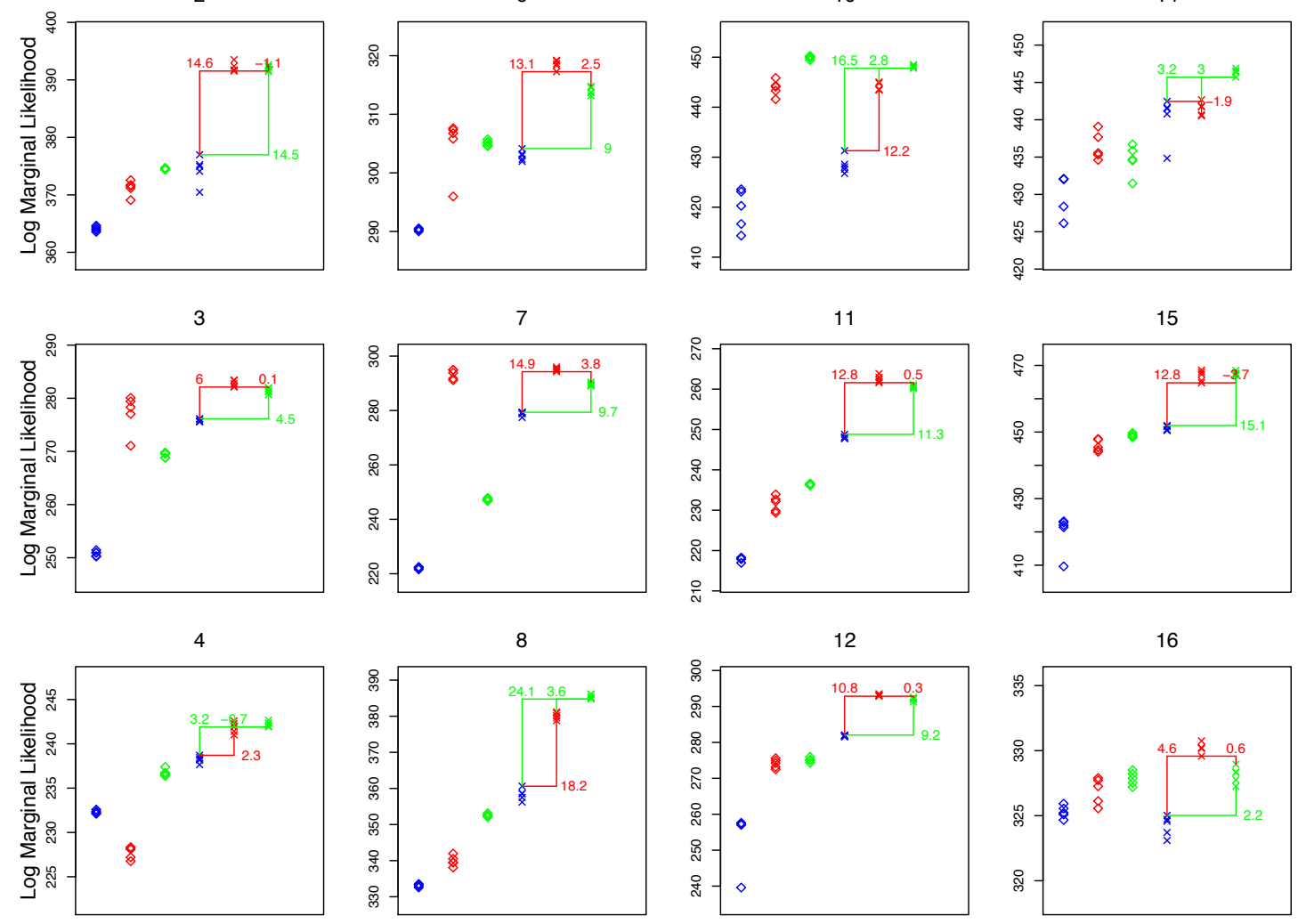

Figure 2. Bayes factor analysis for the Ulrich et al. (2015) data set. 
Table 7: The marginal likelihoods in the Ulrich et al. (2015) data set for the simple DMC, for all 5 estimation runs (columns) of all participants (rows).

\begin{tabular}{lrrrrr}
\hline Subject & 1 & 2 & 3 & 4 & 5 \\
\hline 1 & 350.38 & 350.54 & 350.00 & 350.97 & 351.59 \\
2 & 364.64 & 364.38 & 363.84 & 364.08 & 363.56 \\
3 & 250.32 & 251.43 & 250.23 & 250.96 & 250.93 \\
4 & 232.37 & 232.16 & 232.30 & 232.11 & 232.58 \\
5 & 303.37 & 303.28 & 302.32 & 303.33 & 302.19 \\
6 & 290.41 & 290.02 & 290.27 & 290.40 & 290.53 \\
7 & 222.31 & 222.08 & 222.13 & 221.57 & 222.50 \\
8 & 332.57 & 332.77 & 333.39 & 333.54 & 333.17 \\
9 & 292.23 & 300.22 & 299.90 & 299.78 & 292.60 \\
10 & 423.11 & 423.58 & 420.27 & 414.32 & 416.65 \\
11 & 217.99 & 216.95 & 218.26 & 218.10 & 217.88 \\
12 & 257.15 & 257.61 & 239.61 & 257.06 & 257.27 \\
13 & 434.81 & 433.13 & 439.89 & 433.78 & 441.47 \\
14 & 432.08 & 428.38 & 426.13 & 432.03 & 432.07 \\
15 & 422.82 & 423.19 & 409.64 & 421.40 & 422.02 \\
16 & 324.67 & 325.92 & 325.10 & 325.22 & 325.55 \\
\hline
\end{tabular}


Table 8: The marginal likelihoods in the Ulrich et al. (2015) data set for the complex DMC, for all 5 estimation runs (columns) of all participants (rows).

\begin{tabular}{lrrrrr}
\hline Subject & 1 & 2 & 3 & 4 & 5 \\
\hline 1 & 373.68 & 373.50 & 373.25 & 372.96 & 373.24 \\
2 & 370.45 & 374.96 & 375.25 & 374.10 & 376.97 \\
3 & 276.15 & 275.55 & 275.66 & 275.72 & 276.09 \\
4 & 238.23 & 238.17 & 238.71 & 237.65 & 238.45 \\
5 & 322.51 & 323.34 & 323.44 & 323.96 & 324.01 \\
6 & 302.99 & 302.34 & 303.21 & 304.14 & 301.96 \\
7 & 279.31 & 278.79 & 279.36 & 279.26 & 277.45 \\
8 & 358.53 & 358.62 & 356.22 & 360.62 & 357.50 \\
9 & 356.33 & 357.11 & 356.48 & 356.87 & 356.29 \\
10 & 426.75 & 428.07 & 427.59 & 428.58 & 431.30 \\
11 & 247.79 & 248.18 & 248.08 & 247.77 & 248.80 \\
12 & 282.05 & 281.77 & 281.75 & 281.53 & 281.95 \\
13 & 450.74 & 446.41 & 446.34 & 439.52 & 449.04 \\
14 & 442.46 & 434.83 & 441.61 & 441.49 & 440.77 \\
15 & 451.96 & 450.47 & 450.56 & 450.72 & 451.63 \\
16 & 323.10 & 324.72 & 323.71 & 325.02 & 324.58 \\
\hline
\end{tabular}


Table 9: The marginal likelihoods in the Ulrich et al. (2015) data set for the simple DSTP, for all 5 estimation runs (columns) of all participants (rows).

\begin{tabular}{lrrrrr}
\hline Subject & 1 & 2 & 3 & 4 & 5 \\
\hline 1 & 360.67 & 361.88 & 359.59 & 357.80 & 359.64 \\
2 & 372.56 & 371.16 & 371.72 & 369.08 & 371.52 \\
3 & 279.41 & 280.08 & 277.05 & 278.32 & 271.06 \\
4 & 226.75 & 228.20 & 228.34 & 227.20 & 228.10 \\
5 & 315.18 & 318.71 & 319.10 & 318.45 & 321.24 \\
6 & 306.76 & 307.36 & 305.82 & 295.97 & 307.62 \\
7 & 291.74 & 294.93 & 294.95 & 291.14 & 293.88 \\
8 & 339.49 & 340.51 & 338.09 & 339.44 & 341.95 \\
9 & 352.63 & 355.80 & 354.02 & 359.17 & 355.75 \\
10 & 445.85 & 443.30 & 444.28 & 444.08 & 441.58 \\
11 & 232.69 & 229.37 & 232.17 & 233.92 & 229.86 \\
12 & 275.61 & 273.15 & 274.23 & 274.92 & 272.51 \\
13 & 454.60 & 455.17 & 456.20 & 454.86 & 454.21 \\
14 & 435.32 & 435.55 & 439.09 & 437.67 & 434.62 \\
15 & 444.59 & 445.40 & 447.77 & 444.05 & 447.90 \\
16 & 325.56 & 327.73 & 326.13 & 327.26 & 327.90 \\
\hline
\end{tabular}


Table 10: The marginal likelihoods in the Ulrich et al. (2015) data set for the complex DSTP, for all 5 estimation runs (columns) of all participants (rows).

\begin{tabular}{lrrrrr}
\hline Subject & 1 & 2 & 3 & 4 & 5 \\
\hline 1 & 386.95 & 384.83 & 386.58 & 385.86 & 387.45 \\
2 & 392.36 & 391.70 & 393.51 & 391.54 & 391.71 \\
3 & 282.77 & 282.27 & 283.31 & 283.44 & 282.10 \\
4 & 241.89 & 242.59 & 242.28 & 241.02 & 241.42 \\
5 & 329.81 & 328.84 & 329.88 & 329.69 & 330.13 \\
6 & 319.23 & 318.64 & 318.34 & 319.08 & 317.27 \\
7 & 295.43 & 296.00 & 294.74 & 294.26 & 295.06 \\
8 & 379.53 & 380.89 & 380.15 & 378.80 & 381.16 \\
9 & 365.71 & 364.52 & 365.01 & 365.52 & 365.09 \\
10 & 445.04 & 443.46 & 443.56 & 443.49 & 444.83 \\
11 & 262.93 & 262.34 & 261.59 & 263.85 & 261.84 \\
12 & 293.52 & 293.09 & 293.25 & 292.85 & 292.95 \\
13 & 464.47 & 465.03 & 464.06 & 464.56 & 464.96 \\
14 & 442.67 & 440.51 & 441.74 & 440.68 & 441.82 \\
15 & 464.77 & 465.44 & 467.95 & 467.34 & 468.61 \\
16 & 330.22 & 329.58 & 330.21 & 330.75 & 330.18 \\
\hline
\end{tabular}


Table 11: The marginal likelihoods in the Ulrich et al. (2015) data set for the simple SSP, for all 5 estimation runs (columns) of all participants (rows).

\begin{tabular}{lrrrrr}
\hline Subject & 1 & 2 & 3 & 4 & 5 \\
\hline 1 & 361.97 & 361.82 & 362.23 & 362.33 & 361.17 \\
2 & 374.44 & 374.40 & 374.39 & 374.70 & 374.63 \\
3 & 269.83 & 268.80 & 269.56 & 268.83 & 269.59 \\
4 & 236.38 & 237.39 & 236.74 & 236.60 & 236.55 \\
5 & 312.41 & 312.43 & 312.20 & 312.43 & 312.32 \\
6 & 305.31 & 304.57 & 304.73 & 305.73 & 305.17 \\
7 & 247.68 & 247.86 & 246.74 & 247.35 & 247.44 \\
8 & 352.44 & 352.64 & 353.14 & 352.21 & 352.14 \\
9 & 334.41 & 335.20 & 334.92 & 334.76 & 334.49 \\
10 & 449.85 & 449.91 & 450.25 & 450.18 & 449.43 \\
11 & 236.36 & 236.42 & 236.34 & 236.66 & 236.13 \\
12 & 275.95 & 275.20 & 275.07 & 274.30 & 275.01 \\
13 & 447.54 & 447.51 & 447.27 & 447.43 & 447.31 \\
14 & 435.87 & 436.69 & 434.55 & 434.69 & 431.48 \\
15 & 449.77 & 449.17 & 448.55 & 448.90 & 448.40 \\
16 & 328.21 & 327.19 & 327.53 & 328.50 & 327.88 \\
\hline
\end{tabular}


Table 12: The marginal likelihoods in the Ulrich et al. (2015) data set for the complex SSP, for all 5 estimation runs (columns) of all participants (rows).

\begin{tabular}{lrrrrr}
\hline Subject & 1 & 2 & 3 & 4 & 5 \\
\hline 1 & 385.97 & 386.79 & 385.79 & 386.08 & 386.11 \\
2 & 392.20 & 391.73 & 391.45 & 392.32 & 392.61 \\
3 & 281.20 & 281.82 & 280.68 & 282.00 & 281.36 \\
4 & 242.01 & 242.31 & 242.67 & 241.91 & 242.34 \\
5 & 326.81 & 326.19 & 325.98 & 326.50 & 325.39 \\
6 & 314.57 & 313.18 & 314.78 & 313.69 & 313.72 \\
7 & 289.79 & 289.03 & 290.49 & 289.14 & 289.58 \\
8 & 386.18 & 385.00 & 386.04 & 385.32 & 384.76 \\
9 & 363.70 & 363.39 & 362.09 & 364.14 & 363.87 \\
10 & 448.29 & 447.79 & 447.95 & 448.29 & 448.52 \\
11 & 260.68 & 260.76 & 260.13 & 261.10 & 260.36 \\
12 & 292.03 & 291.24 & 292.35 & 292.52 & 291.98 \\
13 & 455.58 & 455.58 & 456.77 & 456.15 & 455.60 \\
14 & 446.57 & 446.45 & 445.71 & 446.38 & 446.90 \\
15 & 468.51 & 467.38 & 467.73 & 467.35 & 467.11 \\
16 & 327.75 & 327.25 & 328.33 & 328.35 & 328.95 \\
\hline
\end{tabular}



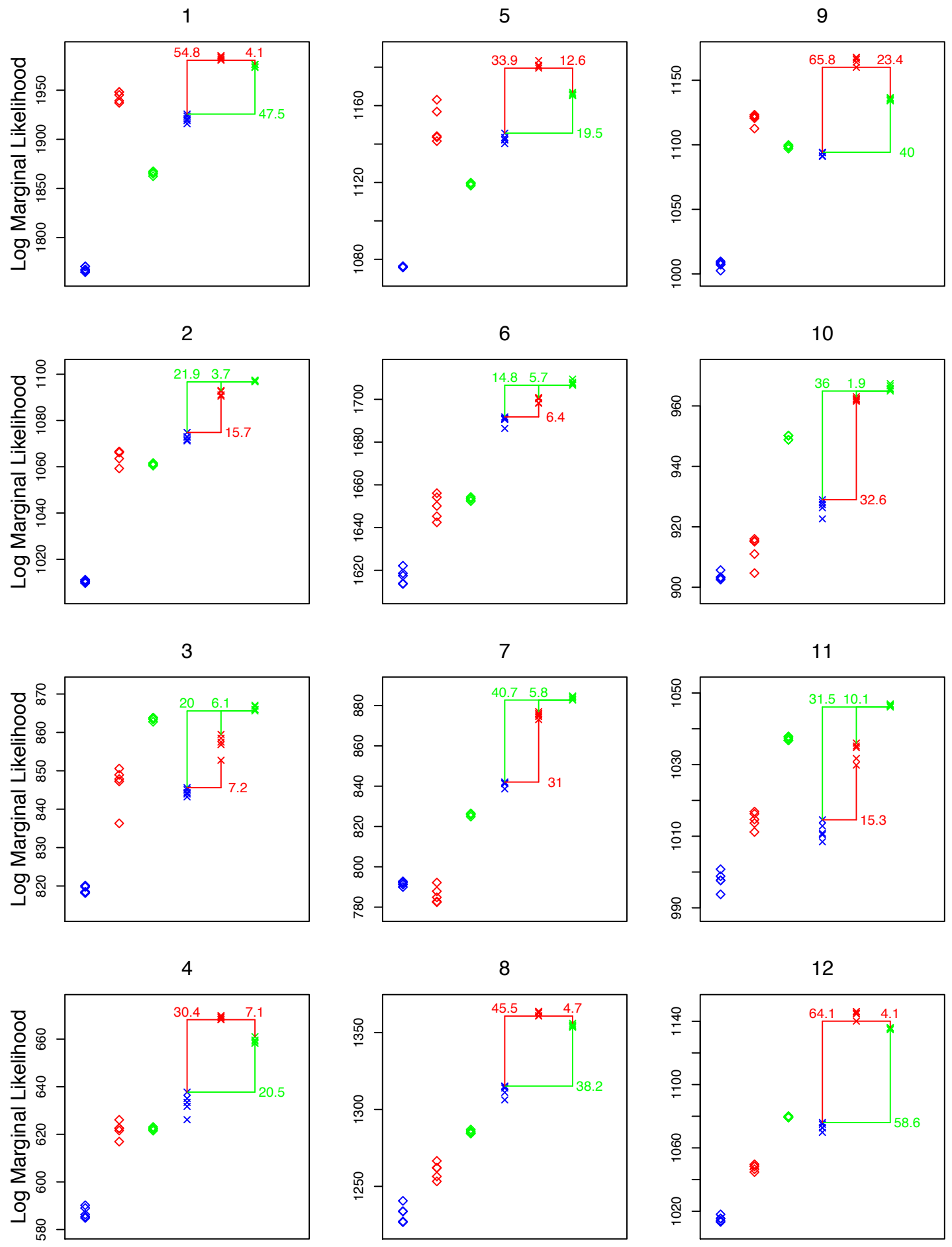

Figure 3. Bayes factor analysis for the Servant et al. (2015) data set. 
Table 13: The marginal likelihoods in the Servant et al. (2015) data set for the simple DMC, for all 5 estimation runs (columns) of all participants (rows).

\begin{tabular}{lrrrrr}
\hline Subject & 1 & 2 & 3 & 4 & 5 \\
\hline 1 & 1764.74 & 1767.59 & 1770.60 & 1766.19 & 1770.55 \\
2 & 1010.48 & 1010.23 & 1010.99 & 1009.70 & 1011.15 \\
3 & 818.30 & 818.15 & 820.11 & 819.76 & 818.49 \\
4 & 584.86 & 589.02 & 585.39 & 586.28 & 590.19 \\
5 & 1075.74 & 1076.00 & 1076.14 & 1076.06 & 1076.42 \\
6 & 1613.90 & 1618.61 & 1617.51 & 1622.15 & 1613.61 \\
7 & 792.78 & 792.14 & 791.26 & 789.96 & 791.25 \\
8 & 1226.71 & 1233.90 & 1227.19 & 1240.56 & 1233.62 \\
9 & 1007.13 & 1002.53 & 1009.74 & 1008.63 & 1007.46 \\
10 & 905.67 & 903.09 & 903.36 & 903.15 & 902.54 \\
11 & 997.70 & 998.84 & 993.76 & 1000.77 & 997.66 \\
12 & 1013.96 & 1018.00 & 1015.29 & 1013.24 & 1015.49 \\
\hline
\end{tabular}


Table 14: The marginal likelihoods in the Servant et al. (2015) data set for the complex DMC, for all 5 estimation runs (columns) of all participants (rows).

\begin{tabular}{lrrrrr}
\hline Subject & 1 & 2 & 3 & 4 & 5 \\
\hline 1 & 1919.14 & 1920.89 & 1925.66 & 1923.54 & 1915.87 \\
2 & 1071.67 & 1072.99 & 1072.82 & 1071.19 & 1074.84 \\
3 & 844.32 & 845.07 & 845.60 & 843.20 & 843.98 \\
4 & 637.73 & 631.74 & 633.43 & 626.14 & 635.06 \\
5 & 1143.45 & 1140.25 & 1142.13 & 1142.46 & 1145.66 \\
6 & 1690.57 & 1686.40 & 1691.80 & 1690.95 & 1691.17 \\
7 & 841.43 & 842.03 & 841.25 & 841.42 & 838.63 \\
8 & 1314.90 & 1311.11 & 1315.25 & 1313.96 & 1306.23 \\
9 & 1094.23 & 1090.97 & 1094.00 & 1093.82 & 1091.44 \\
10 & 922.63 & 926.32 & 928.98 & 928.12 & 927.33 \\
11 & 1012.86 & 1008.44 & 1010.83 & 1014.59 & 1010.42 \\
12 & 1072.60 & 1069.83 & 1072.77 & 1076.02 & 1074.91 \\
\hline
\end{tabular}


Table 15: The marginal likelihoods in the Servant et al. (2015) data set for the simple DSTP, for all 5 estimation runs (columns) of all participants (rows).

\begin{tabular}{lrrrrr}
\hline Subject & 1 & 2 & 3 & 4 & 5 \\
\hline 1 & 1937.13 & 1948.05 & 1948.23 & 1945.19 & 1939.18 \\
2 & 1066.52 & 1066.15 & 1059.26 & 1066.60 & 1063.53 \\
3 & 847.24 & 848.96 & 836.31 & 850.59 & 847.86 \\
4 & 626.09 & 621.61 & 616.93 & 622.52 & 622.53 \\
5 & 1143.54 & 1141.52 & 1163.07 & 1144.11 & 1156.87 \\
6 & 1656.11 & 1654.17 & 1650.12 & 1642.45 & 1645.31 \\
7 & 784.84 & 782.79 & 792.19 & 782.45 & 787.86 \\
8 & 1256.46 & 1262.20 & 1253.25 & 1261.99 & 1266.55 \\
9 & 1112.63 & 1121.61 & 1122.23 & 1123.29 & 1120.59 \\
10 & 915.08 & 904.69 & 915.37 & 911.00 & 915.99 \\
11 & 1013.68 & 1016.87 & 1016.27 & 1011.18 & 1014.68 \\
12 & 1048.49 & 1044.72 & 1048.28 & 1049.66 & 1046.52 \\
\hline
\end{tabular}


Table 16: The marginal likelihoods in the Servant et al. (2015) data set for the complex DSTP, for all 5 estimation runs (columns) of all participants (rows).

\begin{tabular}{lrrrrr}
\hline Subject & 1 & 2 & 3 & 4 & 5 \\
\hline 1 & 1984.94 & 1983.35 & 1985.02 & 1981.48 & 1980.45 \\
2 & 1092.97 & 1090.53 & 1092.63 & 1091.08 & 1092.69 \\
3 & 852.75 & 859.49 & 856.81 & 858.50 & 857.48 \\
4 & 668.15 & 668.25 & 669.89 & 668.94 & 669.32 \\
5 & 1179.52 & 1180.48 & 1180.78 & 1183.49 & 1181.00 \\
6 & 1700.56 & 1698.16 & 1698.34 & 1700.27 & 1700.87 \\
7 & 874.48 & 873.03 & 876.94 & 875.28 & 876.01 \\
8 & 1360.74 & 1363.57 & 1362.86 & 1363.84 & 1361.02 \\
9 & 1167.75 & 1164.03 & 1167.48 & 1160.02 & 1166.20 \\
10 & 962.40 & 961.54 & 962.18 & 961.93 & 963.05 \\
11 & 1036.00 & 1031.71 & 1035.20 & 1034.76 & 1029.86 \\
12 & 1145.02 & 1146.12 & 1146.10 & 1140.07 & 1144.63 \\
\hline
\end{tabular}


Table 17: The marginal likelihoods in the Servant et al. (2015) data set for the simple SSP, for all 5 estimation runs (columns) of all participants (rows).

\begin{tabular}{lrrrrr}
\hline Subject & 1 & 2 & 3 & 4 & 5 \\
\hline 1 & 1867.24 & 1862.40 & 1864.92 & 1865.73 & 1867.43 \\
2 & 1060.48 & 1061.63 & 1060.86 & 1061.08 & 1061.36 \\
3 & 863.84 & 863.74 & 862.78 & 863.37 & 863.86 \\
4 & 621.54 & 622.43 & 622.23 & 622.31 & 623.09 \\
5 & 1119.16 & 1119.40 & 1119.91 & 1119.71 & 1118.35 \\
6 & 1654.32 & 1653.46 & 1652.39 & 1652.53 & 1653.56 \\
7 & 826.49 & 826.00 & 824.89 & 826.37 & 825.77 \\
8 & 1285.21 & 1285.83 & 1284.34 & 1286.96 & 1284.47 \\
9 & 1099.54 & 1097.04 & 1099.88 & 1098.49 & 1098.50 \\
10 & 950.20 & 950.17 & 948.76 & 948.85 & 950.20 \\
11 & 1036.77 & 1036.74 & 1037.65 & 1037.90 & 1037.20 \\
12 & 1079.15 & 1079.91 & 1079.61 & 1079.22 & 1080.10 \\
\hline
\end{tabular}


Table 18: The marginal likelihoods in the Servant et al. (2015) data set for the complex SSP, for all 5 estimation runs (columns) of all participants (rows).

\begin{tabular}{lrrrrr}
\hline Subject & 1 & 2 & 3 & 4 & 5 \\
\hline 1 & 1976.02 & 1973.92 & 1976.32 & 1976.29 & 1973.11 \\
2 & 1096.99 & 1097.49 & 1097.15 & 1097.23 & 1096.69 \\
3 & 867.07 & 865.95 & 865.59 & 866.78 & 865.98 \\
4 & 658.22 & 661.02 & 659.16 & 658.35 & 659.57 \\
5 & 1166.95 & 1165.50 & 1166.16 & 1165.20 & 1165.53 \\
6 & 1707.98 & 1709.45 & 1706.60 & 1707.44 & 1707.09 \\
7 & 884.65 & 883.83 & 882.72 & 884.64 & 883.01 \\
8 & 1356.06 & 1353.48 & 1354.93 & 1354.20 & 1354.13 \\
9 & 1136.58 & 1134.22 & 1134.19 & 1136.00 & 1135.24 \\
10 & 965.82 & 967.40 & 966.66 & 964.95 & 965.46 \\
11 & 1046.77 & 1046.51 & 1046.41 & 1046.08 & 1046.93 \\
12 & 1135.75 & 1135.24 & 1134.66 & 1135.95 & 1135.86 \\
\hline
\end{tabular}

DMC robustness analyses

As the main conclusion of our study was that DMC provides a poorer explanation of the flanker task than DSTP and SSP, we performed a series of robustness analyses to ensure that this key result held across a range of potential theoretical and methodology factors. Specifically, we tested an additional 4 variants of DMC that differed in some manner to the main definition of DMC, which we detail below in separate sub-sections. Bayes factor plots including all 4 robustness variants of DMC, as well as the main variants of DSTP and SSP for a point of reference, can be seen in Figure 4 for the White et al. (2011) data set, 
Figure 5 for the Ulrich et al. (2015) data set, and Figure 6 for the Servant et al. (2015) data set. In general, our main conclusion that DMC is never the best model, and is in the vast majority of cases the outright worst model, held across these robustness analyses, even though some robustness analyses provide a strong bias towards DMC in the selection process (see the Prior values robustness analysis subsection, shown as the plus symbols in the figures). 

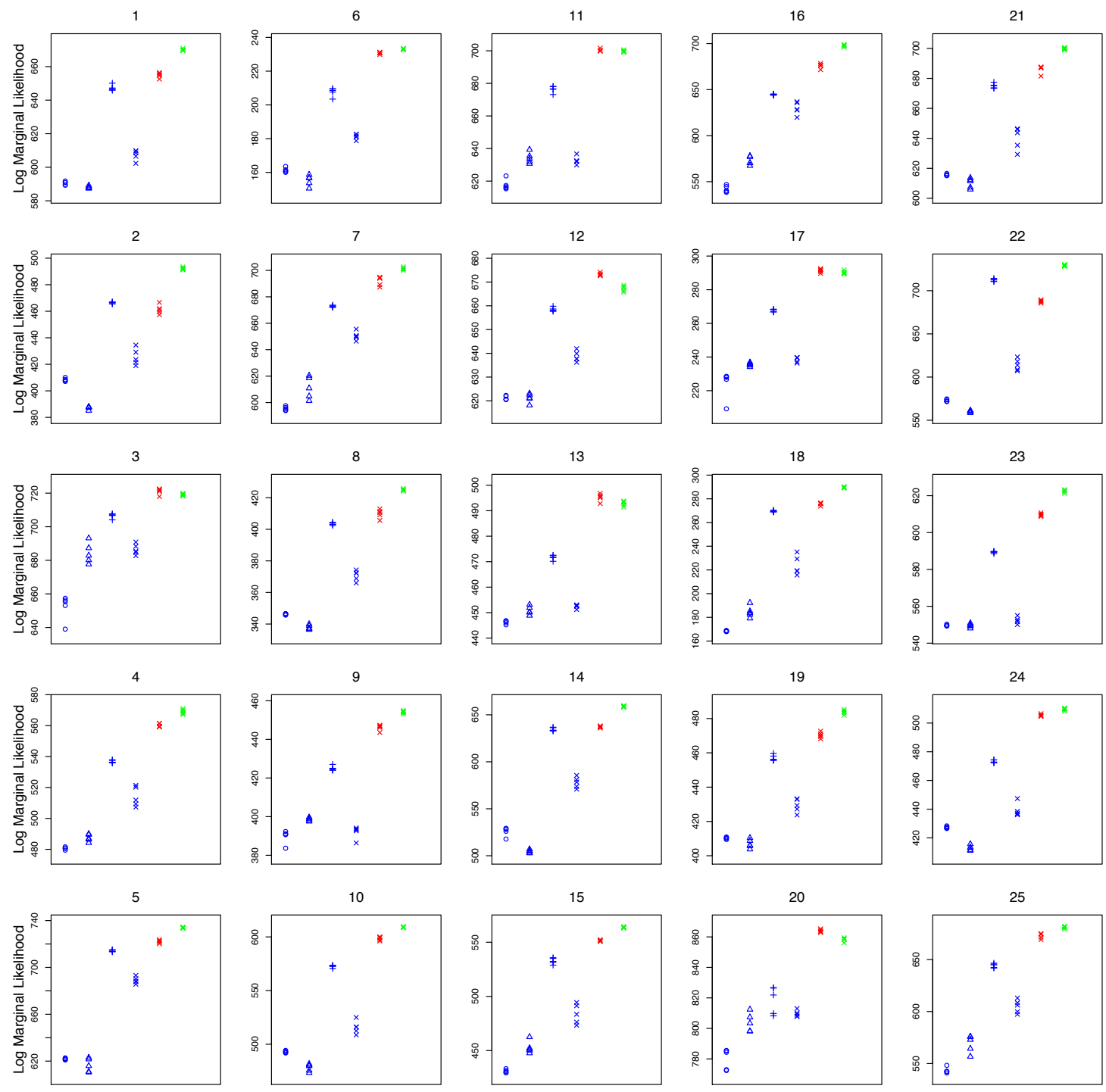

Figure 4. Bayes factor analysis for the White et al. (2011) data set with the 4 additional variants of DMC. As before, DMC is in blue: the circles (first column of each panel) show the $\tau$ prior and starting point analysis, the triangles show the DMC specification analysis, the plus symbols show the empirical Bayes analysis, and the crosses show the time-step analysis. 

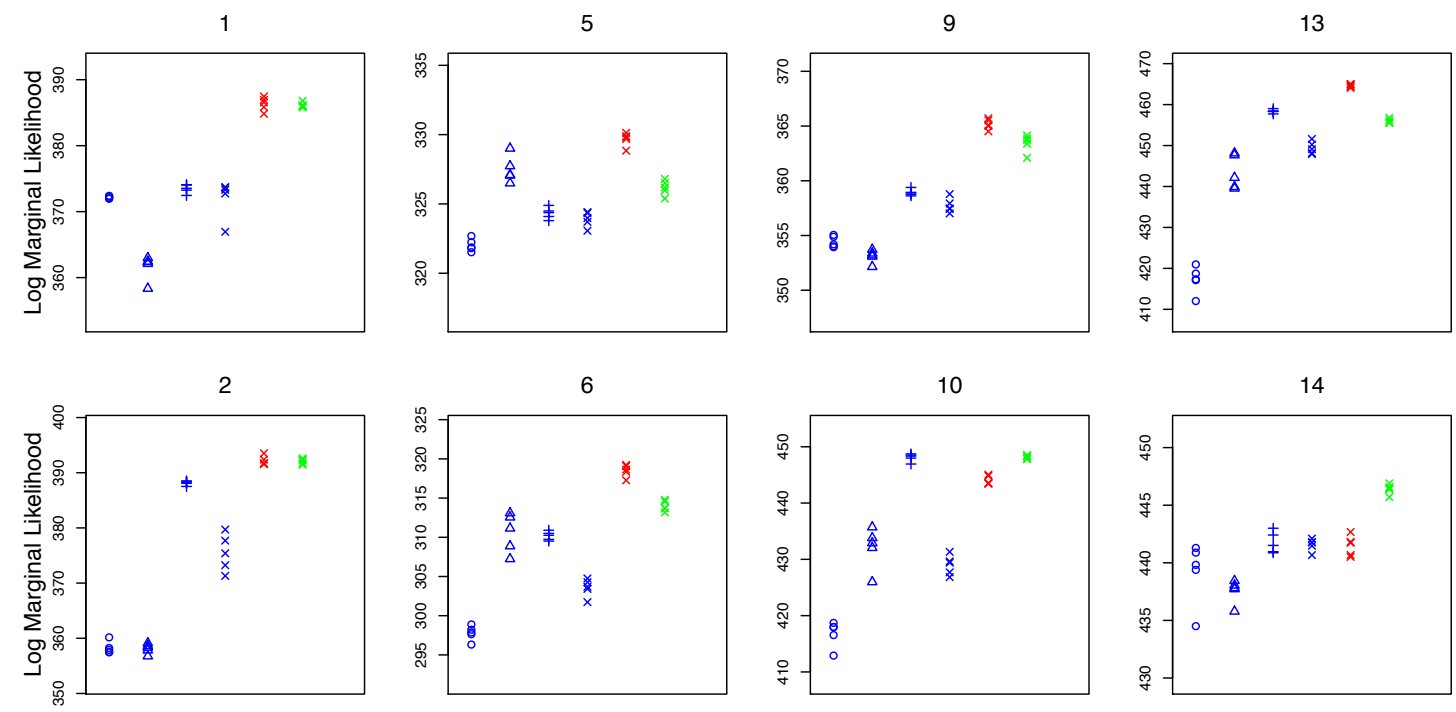

6

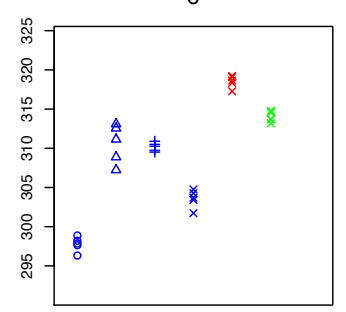

10

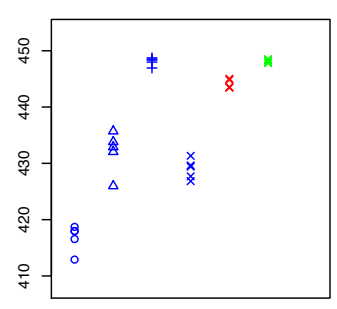

14

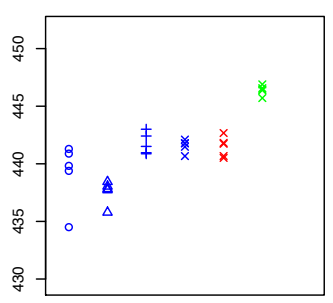

7
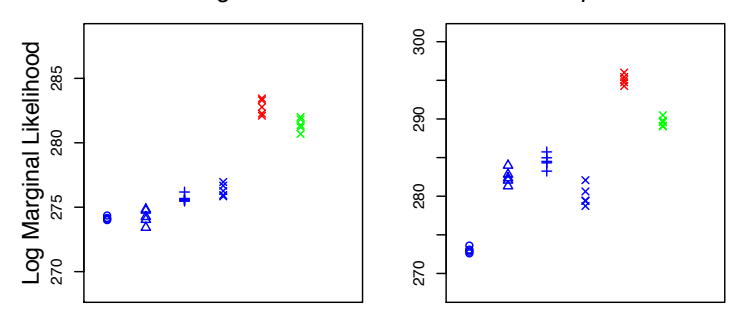

11

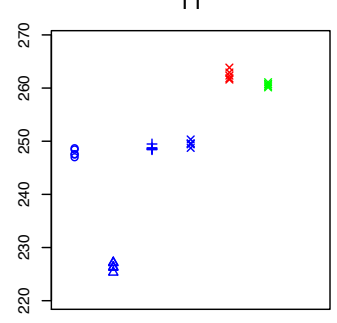

15

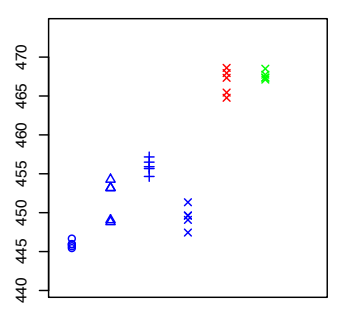

8
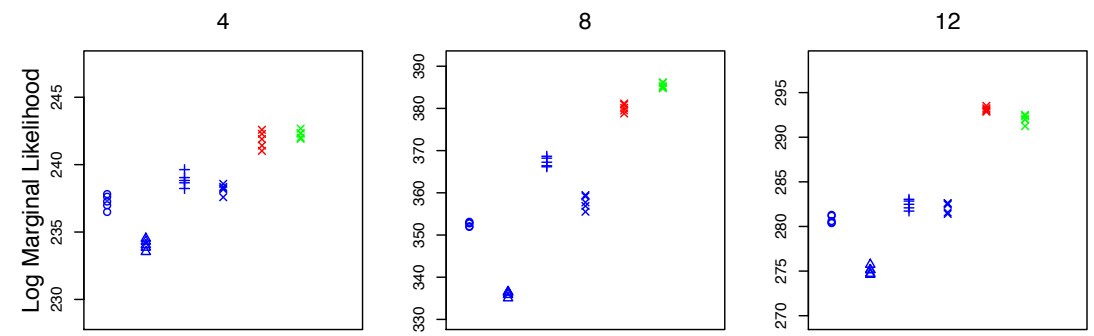

16

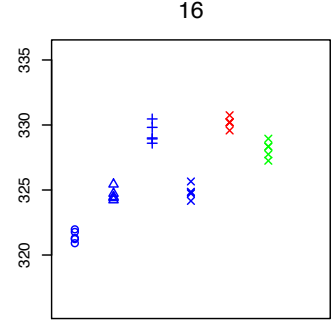

Figure 5. Bayes factor analysis for the Ulrich et al. (2015) data set with the 4 additional variants of DMC. As before, DMC is in blue: the circles (first column of each panel) show the $\tau$ prior and starting point analysis, the triangles show the DMC specification analysis, the plus symbols show the empirical Bayes analysis, and the crosses show the time-step analysis. 
1

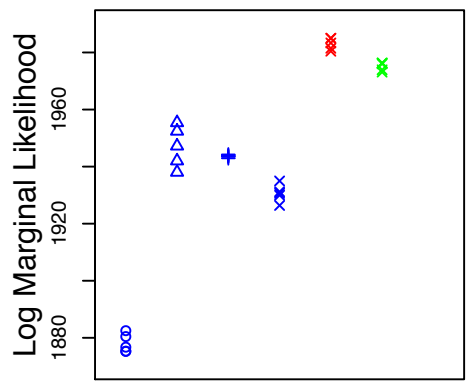

2

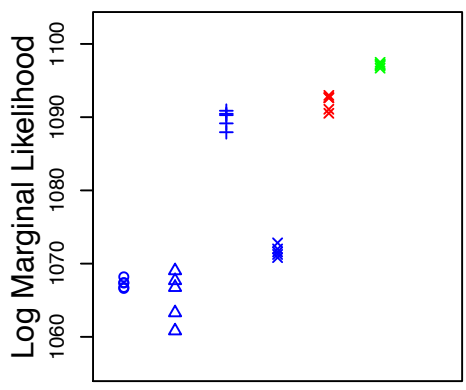

3

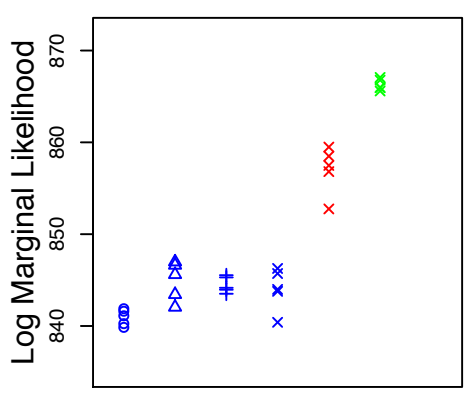

4

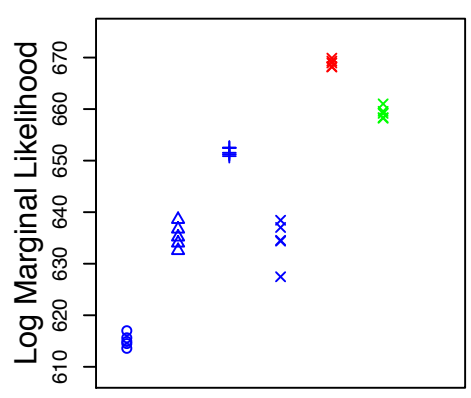

5

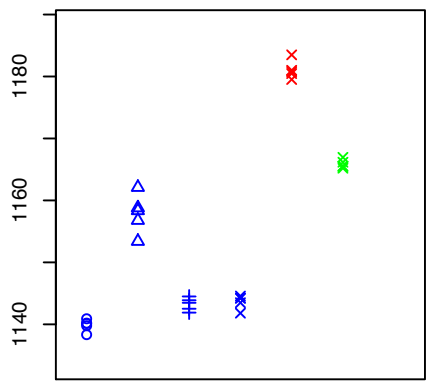

6

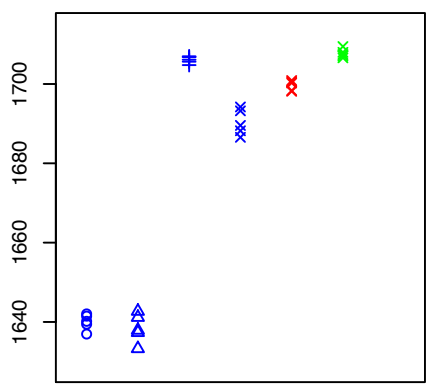

7

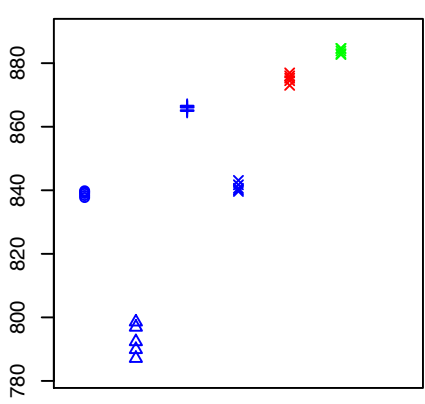

8

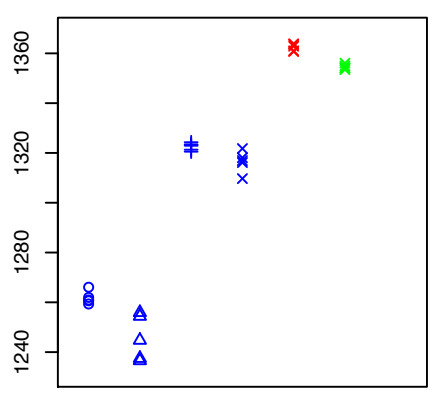

9

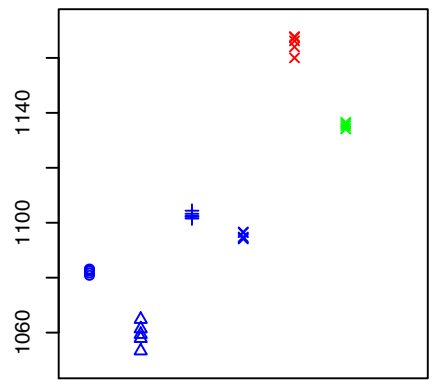

10

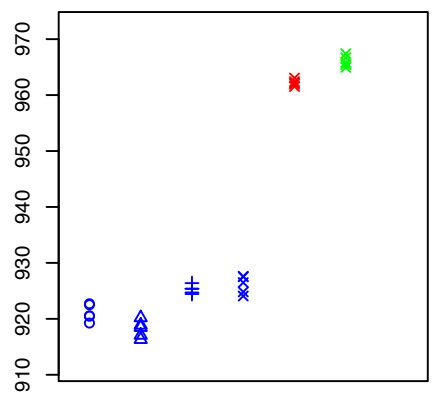

11

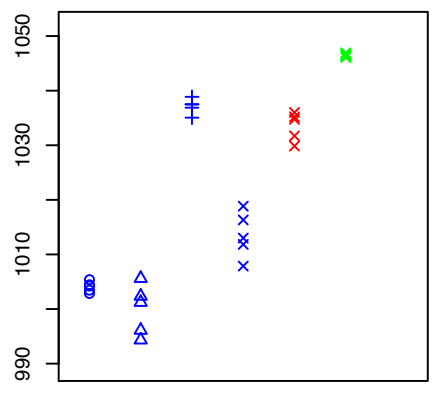

12

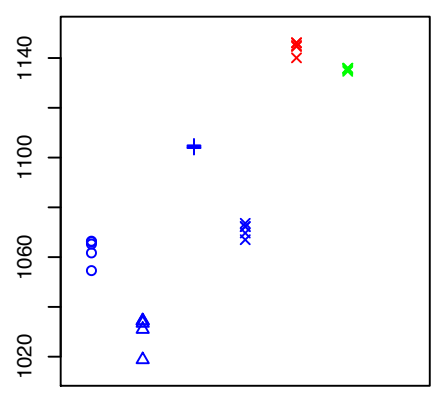

Figure 6. Bayes factor analysis for the Servant et al. (2015) data set with the 4 additional variants of DMC. As before, DMC is in blue: the circles (first column of each panel) show the $\tau$ prior and starting point analysis, the triangles show the DMC specification analysis, the plus symbols show the empirical Bayes analysis, and the crosses show the time-step analysis. 


\section{Model specification robustness analysis}

The first robustness analysis assessed whether our specification of DMC may have influenced our results. Specifically, our study used a definition of DMC that varied slightly from the original definition in Ulrich et al. (2015): while our study used distributions for the between-trial variability parameters that are common within the response time modelling literature, Ulrich et al. (2015) instead used distributions that they believed were more neurologically plausible. Following Ulrich et al. (2015), we defined another version of DMC that contained 1) no between-trial variability in drift rate, 2) a beta distribution for between-trial in starting point that was assumed to be unbiased (i.e., $z=0$ ) and symmetric (i.e., $a=b$ ), and 3) a normal distribution for between-trial variability in non-decision time that also contained a minimum non-decision time (i.e., a truncated normal distribution). Specifically, this removed 4 parameters $\left(z, s_{v}, s_{z}\right.$, and $\left.s_{t e r}\right)$, and added 3 parameters $(\beta$ : the $a$ and $b$ parameters of the starting point beta distribution; $s d_{t e r}$ : the standard deviation

of the non-decision time truncated normal distribution; lowerter: the lower bound of the the non-decision time truncated normal distribution, which we gave the following prior distributions:

$$
\begin{aligned}
\beta & \sim T N(5,5,1, \infty) \\
s d_{t e r} & \sim T N(0.2,0.2,0, \infty) \\
\text { lower }_{\text {ter }} & \sim \operatorname{TN}(0.2,0.2,0, \infty)
\end{aligned}
$$

The pseudo-likelihood Bayes factors for this alternate version of DMC can be seen as the blue triangles in Figures 4, 5, and 6, and the qualitative predictions can be seen as the "DMCoriginal" row in Figures 7, 8, 9, 10, and 11. In most cases this alternate version 
of DMC results in a lower marginal likelihood than our main definition of DMC (though see participants 5 and 6 in the Ulrich et al. data set), and more importantly, this alternate version of DMC does not change our overall pattern of results. 

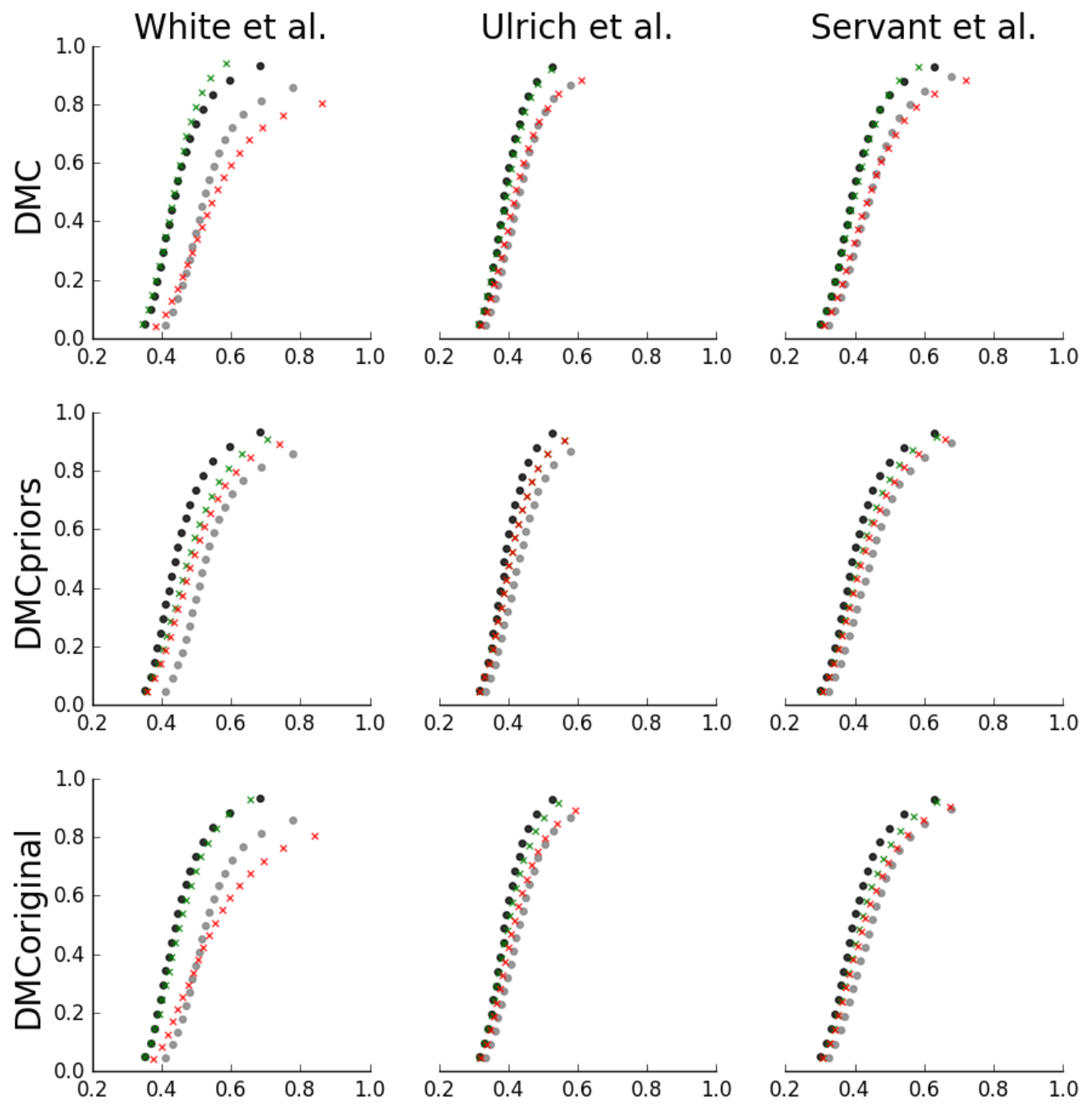

Figure \%. Defective cumulative density function (DCDF) plots for three variants of DMC (rows; our full definition [top], the version with different $\tau$ priors and starting points [middle], and the version based on the original definition of Ulrich et al. [bottom]) in each data set (columns). The DCDF plots display 19 (0.05 to 0.95, in increments of 0.05) response time quantiles (different points) of correct responses for each experimental condition, plotted by the response time (x-axis) and response proportion (y-axis) for that quantile. Dots display observed data, with black dots displaying the compatible condition and grey dots displaying the incompatible condition. Crosses display model predictions, with green crosses displaying the compatible condition and red crosses displaying the incompatible condition. 

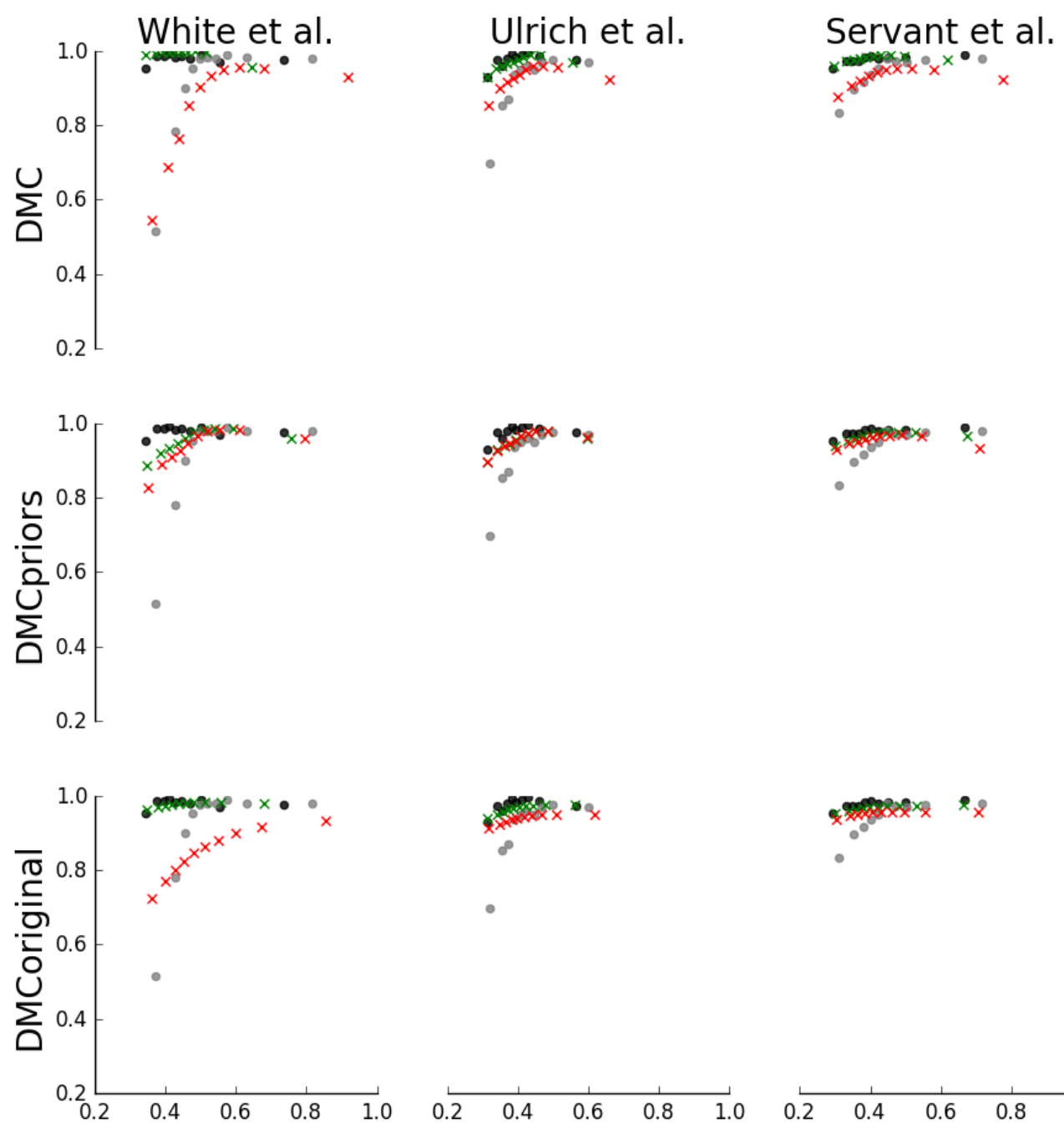

Figure 8. Conditional accuracy function (CAF) plots for three variants of DMC (rows; our full definition [top], the version with different $\tau$ priors and starting points [middle], and the version based on the original definition of Ulrich et al. [bottom]) in each data set (columns). The CAF plots display 10 response time bins (different points), with the upper limit of each bin corresponding to response time quantiles ( 0.1 to 1 , in increments of 0.1 ) for each experimental condition, plotted by the mean response time (x-axis) and response accuracy (y-axis) for that bin. Dots display observed data, with black dots displaying the compatible condition and grey dots displaying the incompatible condition. Crosses display model predictions, with green crosses displaying the compatible condition and red crosses displaying the incompatible condition. 


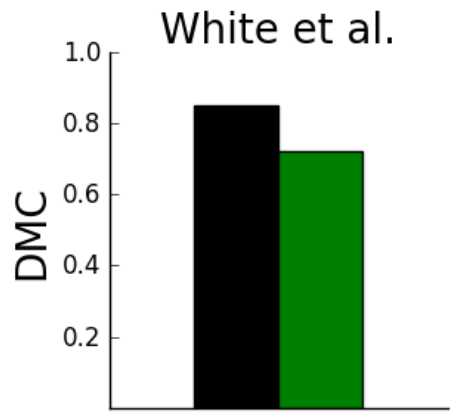

Ulrich et al.

Servant et al.
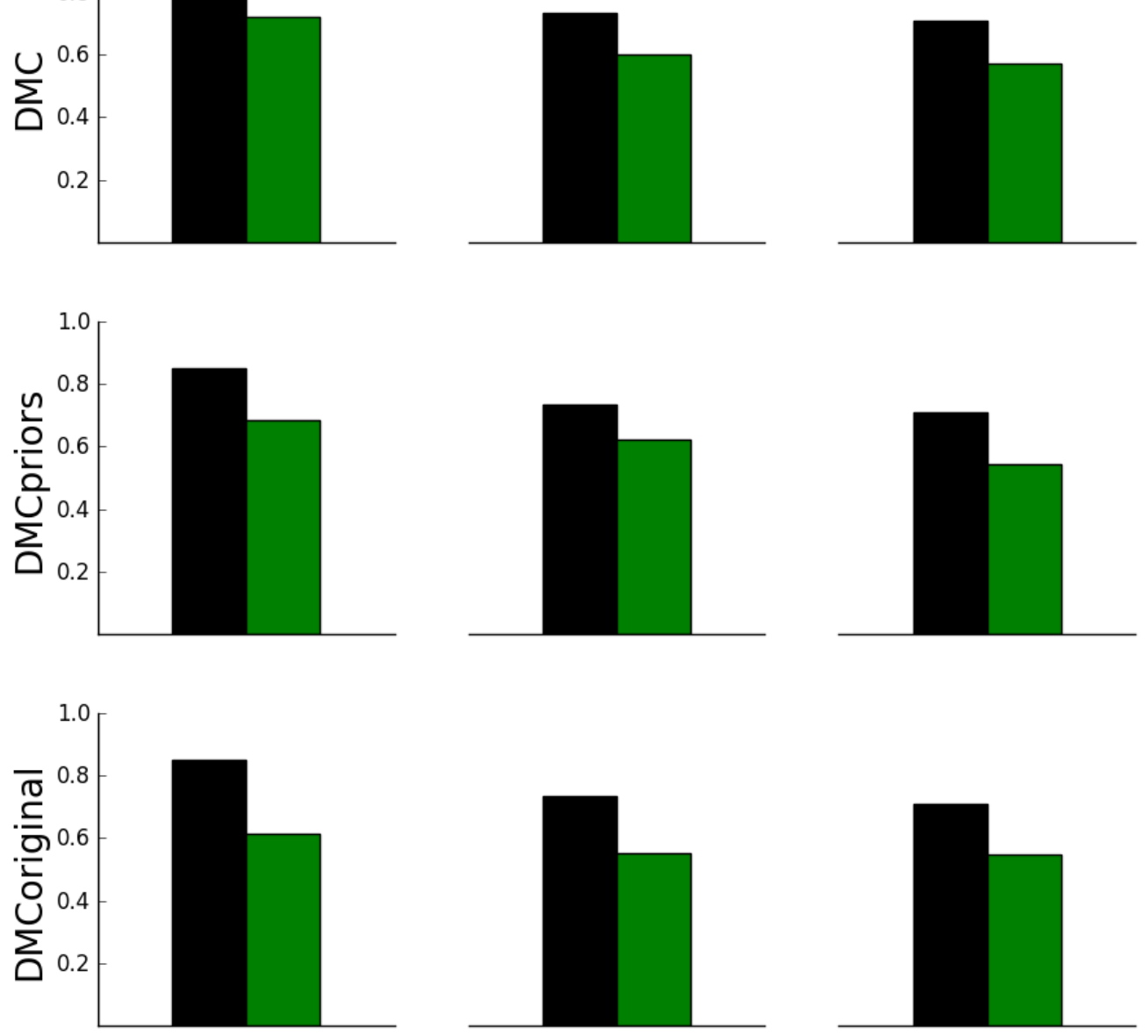

Figure 9. Error location index (ELI) plots for three variants of DMC (rows; our full definition [top], the version with different $\tau$ priors and starting points [middle], and the version based on the original definition of Ulrich et al. [bottom]) in each data set (columns). The ELI plots display the relative speed of correct and error responses in incompatible trials across the entire response time distribution, with a value of 1 indicating that all errors are concentrated among the fastest incompatible responses, and a value of 0 indicating that all errors are concentrated among the slowest incompatible responses (y-axis). Black bars display the observed data, and green bars display the model predictions. 


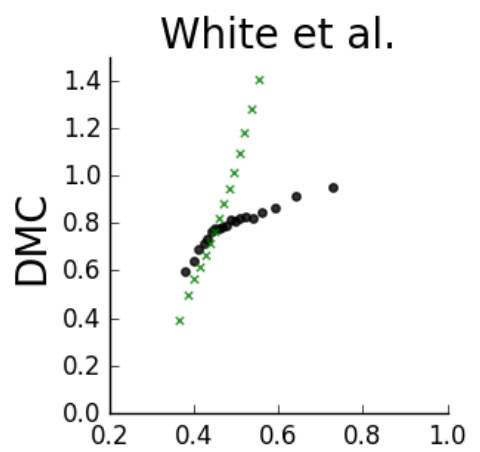

Ulrich et al.

\section{Servant et al.}
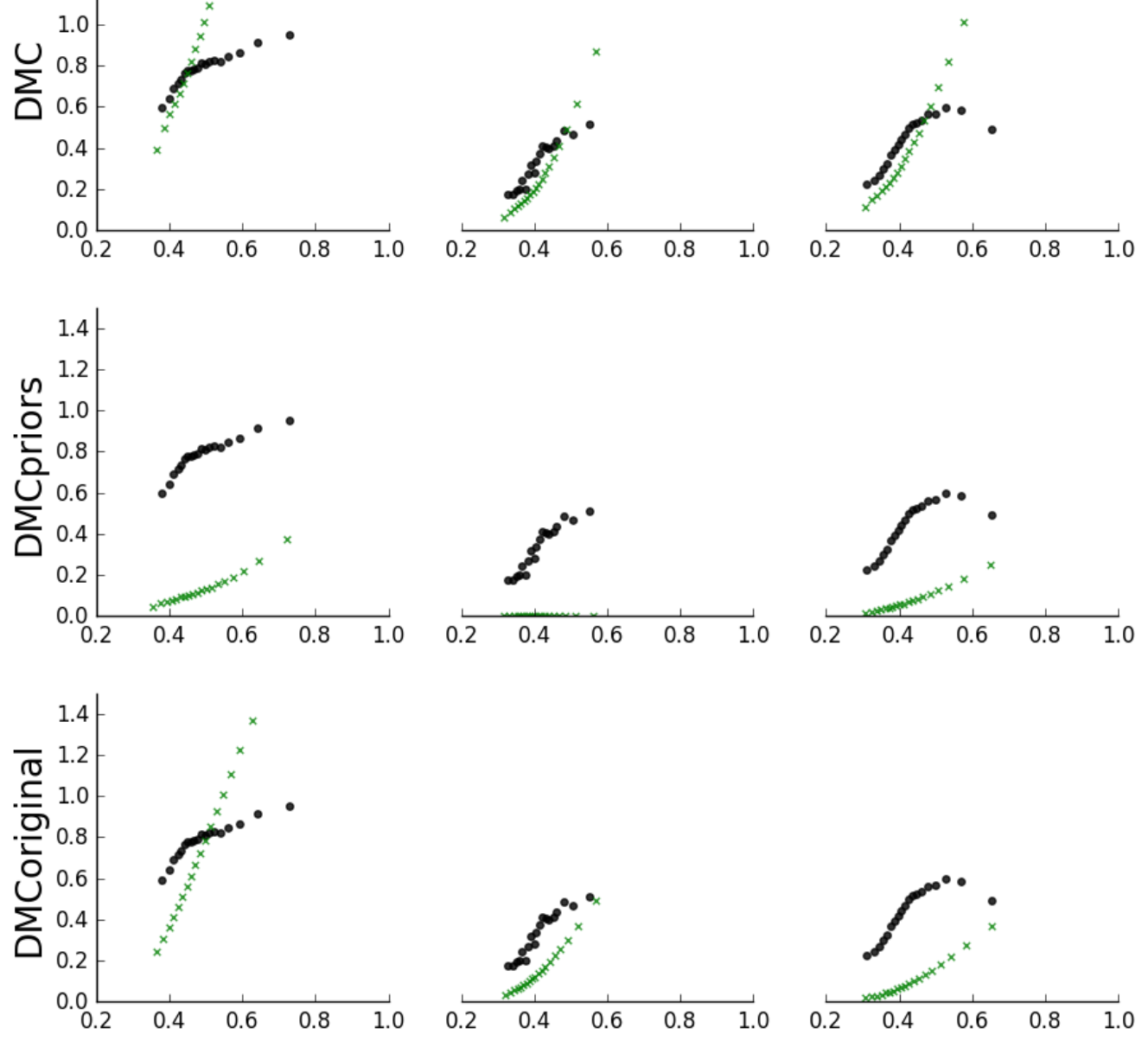

Figure 10. Delta function (DF) plots for three variants of DMC (rows; our full definition [top], the version with different $\tau$ priors and starting points [middle], and the version based on the original definition of Ulrich et al. [bottom]) in each data set (columns). The DF plots display 19 (0.05 to 0.95 , in increments of 0.05 ) response time quantiles (different points) of correct responses for each experimental condition, plotted by the average response time across conditions ( $\mathrm{x}$-axis) and the difference in response time between conditions (y-axis) for that quantile. Black dots represent observed data, and green crosses represent model predictions. 

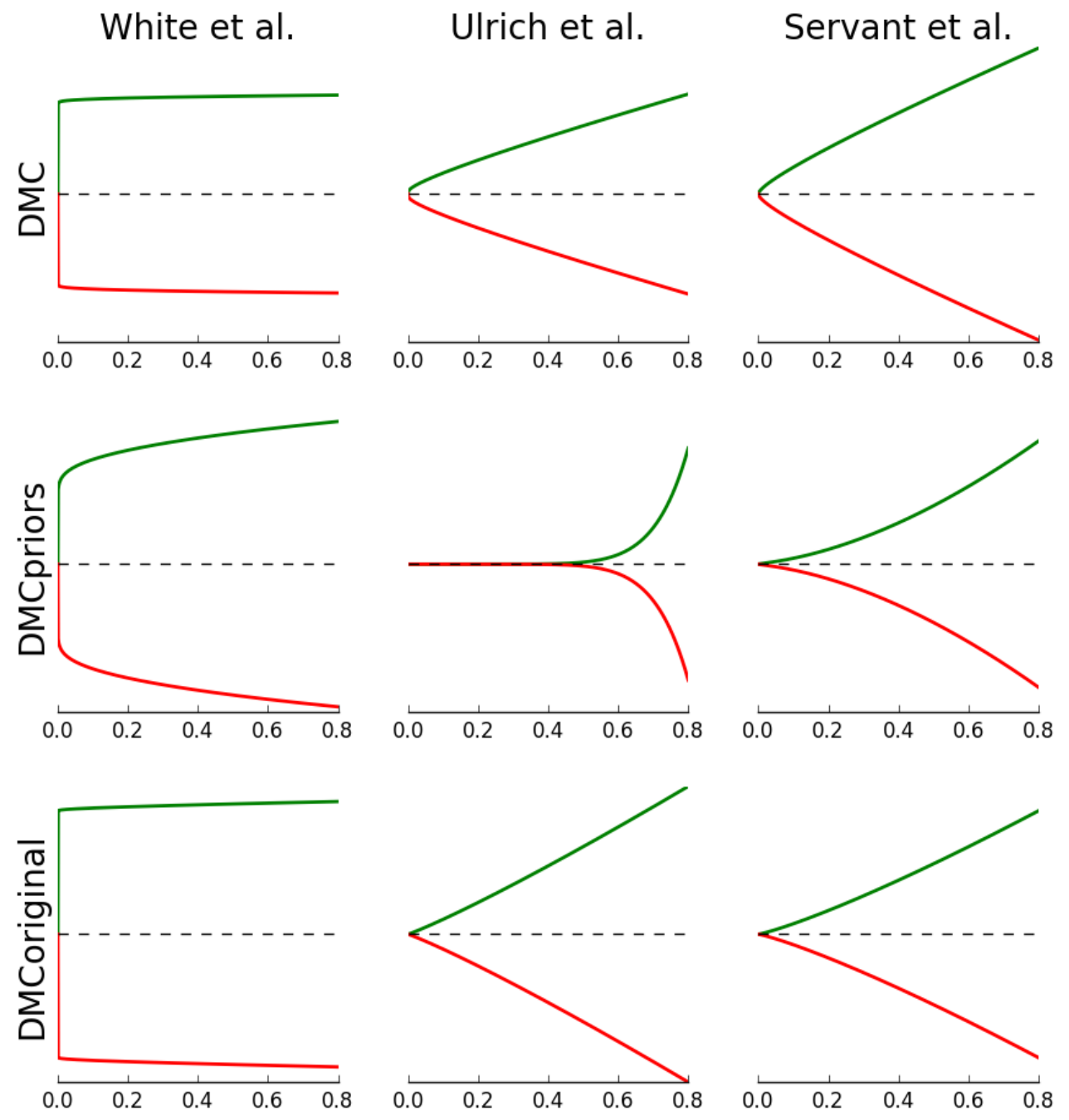

Figure 11. Plots of the time-course of the expected mean of the automatic activation $E\left[X_{a}(t)\right]$ for three variants of DMC (rows; our full definition [top], the version with different $\tau$ priors and starting points [middle], and the version based on the original definition of Ulrich et al. [bottom]) in each data set (columns).The y-axis displays $E\left[X_{a}(t)\right]$, and the x-axis displays the time since accumulation onset. Green lines display the compatible condition, and red lines display the incompatible condition. Black lines indicate the unbiased starting point of accumulation, halfway between the two decision bounds. 
$\tau$ prior and starting point robustness analysis

The second robustness analysis assessed whether our choice of prior and starting point for the $\tau$ parameter may have influenced our results. Specifically, the estimated values of $\tau$ were large for each participant in each data set, which resulted in the expected mean of the automatic activation $E\left[X_{a}(t)\right]$ remaining stable or increasing over the course of the decision, and may have been the result of our choice in prior and/or starting point for $\tau$. Therefore, we re-ran our analyses using the following prior (and starting point) for the $\tau$ parameter, which is closer to values that would result in a mean drift rate for the automatic process that decreases in the latter parts of the decision process:

$$
\tau \sim T N(1,1,0, \infty)
$$

The pseudo-likelihood Bayes factors for DMC with this alternate prior and starting point for $\tau$ can be seen as the blue circles in Figures 4, 5, and 6, and the qualitative predictions can be seen as the "DMCpriors" row in Figures 7, 8, 9, 10, and 11. In the vast majority of cases this alternate prior definition results in a lower marginal likelihood than our main definition of DMC, and more importantly, does not change our overall pattern of results.

Prior values robustness analysis

The third robustness analysis assessed whether our choice of priors for each of DMC's three unique parameters $(\tau, \zeta$, and $\alpha)$ may have influenced the results. Specifically, it is well established that Bayes factors are sensitive to the choice of prior distributions, and in some cases the choice of prior distributions can influence the inferences made within a study. To ensure that the inferiority of DMC in our study was not the result of poorly chosen 
prior distributions for DMC's unique parameters, we re-computed the pseudo-likelihood Bayes factors using an empirical Bayes approach, where the prior mean and variance for the parameters $\tau, \zeta$, and $\alpha$ for each participant in each study were those of the participant's estimated posterior distributions in the hierarchical Bayesian approach. Empirical Bayes approaches have been criticized as general methods of inference, as they use the data for both creating priors and inference (i.e., using the data twice), which consequently means that models that have their priors determined through empirical Bayes have an unfair advantage - when compared in the same data set - over models that have priors determined through other means. However, as a robustness analysis, empirical Bayes provides an extremely strong test of whether our choice of priors for DMC could have had any influence on the results. Importantly, if DMC still shows an inferiority to DSTP and SSP with the "perfect" priors for its unique parameters for each participant in each data set, then this suggests that the inferiority of DMC is due to a fundamental issue in its functional form, and unrelated to our choice of priors.

The empirical Bayes pseudo-likelihood Bayes factors for the full diffusion variant of DMC can be seen as the blue plus symbols in Figures 4, 5, and 6. As expected, in most cases DMC performed better using these "perfect" priors for $\tau, \zeta$, and $\alpha$ than our selected priors. However, even with the empirical Bayes approach, DMC remained generally inferior to both DSTP and SSP, suggesting that our key finding is unrelated to our choice of priors.

\section{Time step robustness analysis}

The final robustness analysis assessed whether our choice of time-step (0.01s) for simulating the models may have influenced our results. For this robustness analysis we re-computed the pseudo-likelihood Bayes factors for the full diffusion variant of DMC using a time-step of $0.001 \mathrm{~s}$, which can be seen as the blue crosses in Figures 4, 5, and

6. However, this change in time-step did not appear to influence the results, with the 
marginal likelihoods for the $0.01 \mathrm{~s}$ and $0.001 \mathrm{~s}$ time-steps being near-identical (i.e., apart from approximation error).

\section{Model recovery assessment}

In addition to our previous robustness analyses, we also performed a model recovery assessment, which involved generating simulated data sets from each of the three models, and then re-fitting each model to each of data set, providing a "cross-fit" of all models to the data generated from each of these models (e.g., White et al., 2018). This allows the assessment of whether the true generating model can be correctly identified, given some method of model selection and some number of models to select between. Importantly, some models are able to better mimic other models than vice versa (e.g., Wagenmakers et al., 2004), meaning that if the better mimicking model was selected for the empirical data, the reasoning would be ambiguous: the better mimicking model could have been selected because it is actually the better model, or because the other model is actually the better model and the better mimicking model was just able to mimic it. Furthermore, there is a possibility that the several layers of approximation within our methodology may have introduced some unexpected bias towards DSTP/SSP or against DMC, which could have influenced our key finding that DSTP and SSP provide a superior explanation of the flanker task than DMC.

We performed two recovery assessments, each with 30 simulated participants per model. The first recovery assessment was based on the data set with the largest number of trials per person within our study, Servant et al. (2015), where we simulated 800 trials per simulated participant per experimental condition, with each participant simulated using the estimated posterior mean of the group-level mean parameters (i.e., each participant was simulated using the same parameter values). The second recovery assessment was based 
on the data set with the smallest number of trials per person within our study, Ulrich et al. (2015), where we simulated 200 trials per simulated participant per experimental condition, with each participant simulated using the estimated posterior mean of the group-level mean parameters.

The results of the recovery assessment can be seen in Figures 12, 13, 14, 15, 16, and 17. In cases where DMC was the true generating model (Figures 12 and 13), DMC was found to be the best model (i.e., highest maximum marginal likelihood) in the vast majority of cases, and there were no cases where DSTP or SSP showed evidence for being a better model than DMC (i.e., whenever DMC was not the best model, the difference was within the approximation error). Importantly, this recovery assessment suggests that our key finding is robust against model mimicry concerns: if DMC were the true model that generated the empirical data sets that we assessed, then we would be unlikely to have found strong evidence for DSTP and/or SSP over DMC in the vast majority of subjects.

The results of the recovery assessment were similar for SSP: when SSP was the true generating model (Figures 14 and 15), SSP was found to be the best model in the vast majority of cases, and there were no cases where DMC or DSTP showed evidence for being a better model than SSP. However, this was not the case for DSTP: when DSTP was the true generating model (Figures 16 and 17), DSTP was found to be an inferior model in many cases, several of which showed evidence for another model over DSTP. Specifically, SSP was found to mimic DSTP in many circumstances, where SSP was found to be a better model than DSTP, and with evidence beyond approximation error. It should also be noted that in cases where DSTP or SSP was the true generating model, DMC was consistently shown to be the worst model, suggesting that DSTP and SSP mimic one another much better than DMC is able to mimic either of these models. 

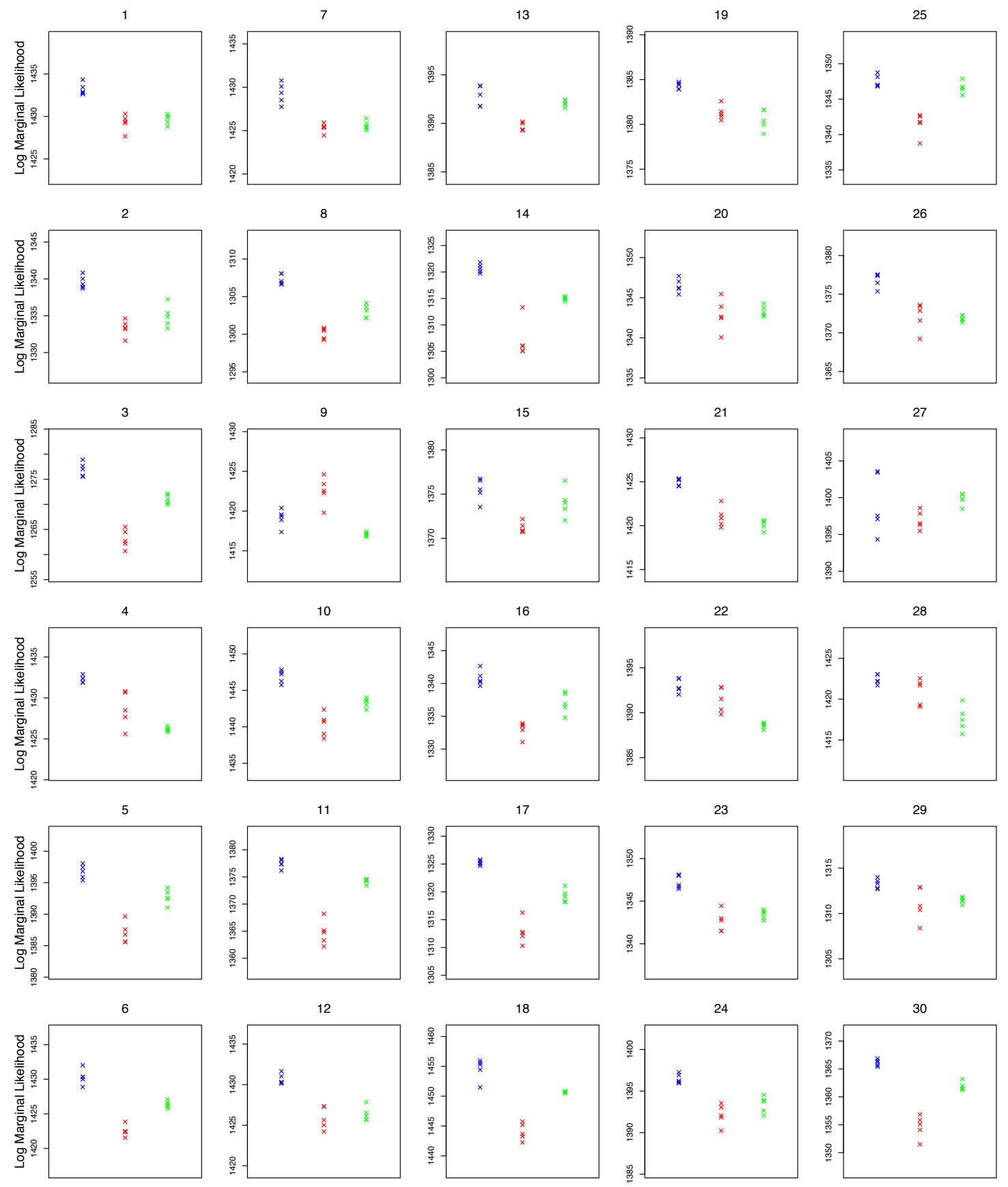

Figure 12. Bayes factor analysis for the recovery analysis. These data are simulated from DMC, based on the data from Servant et al. (2015). 

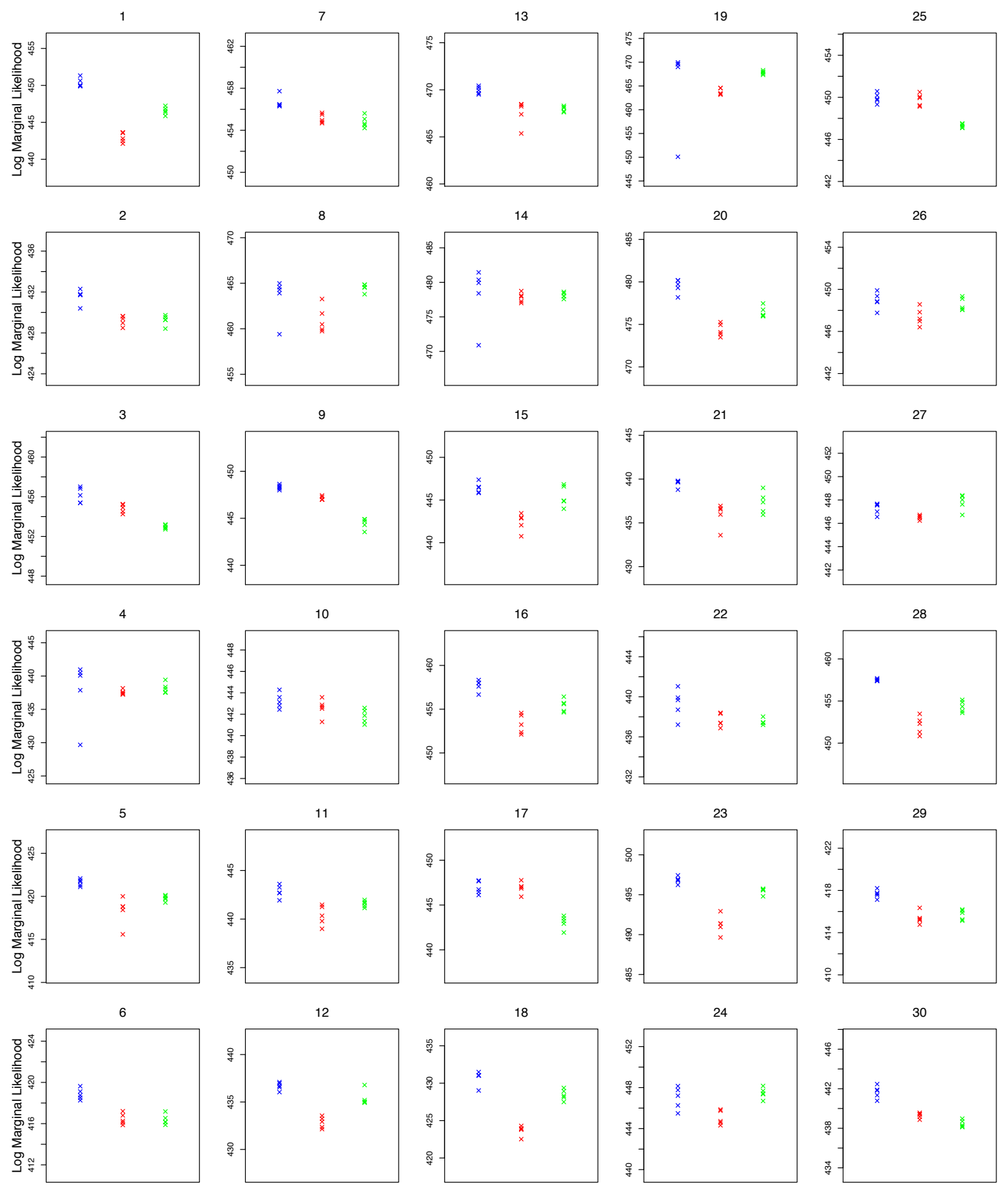

Figure 13. Bayes factor analysis for the recovery analysis. These data are simulated from DMC, based on the data from Ulrich et al. (2015). 

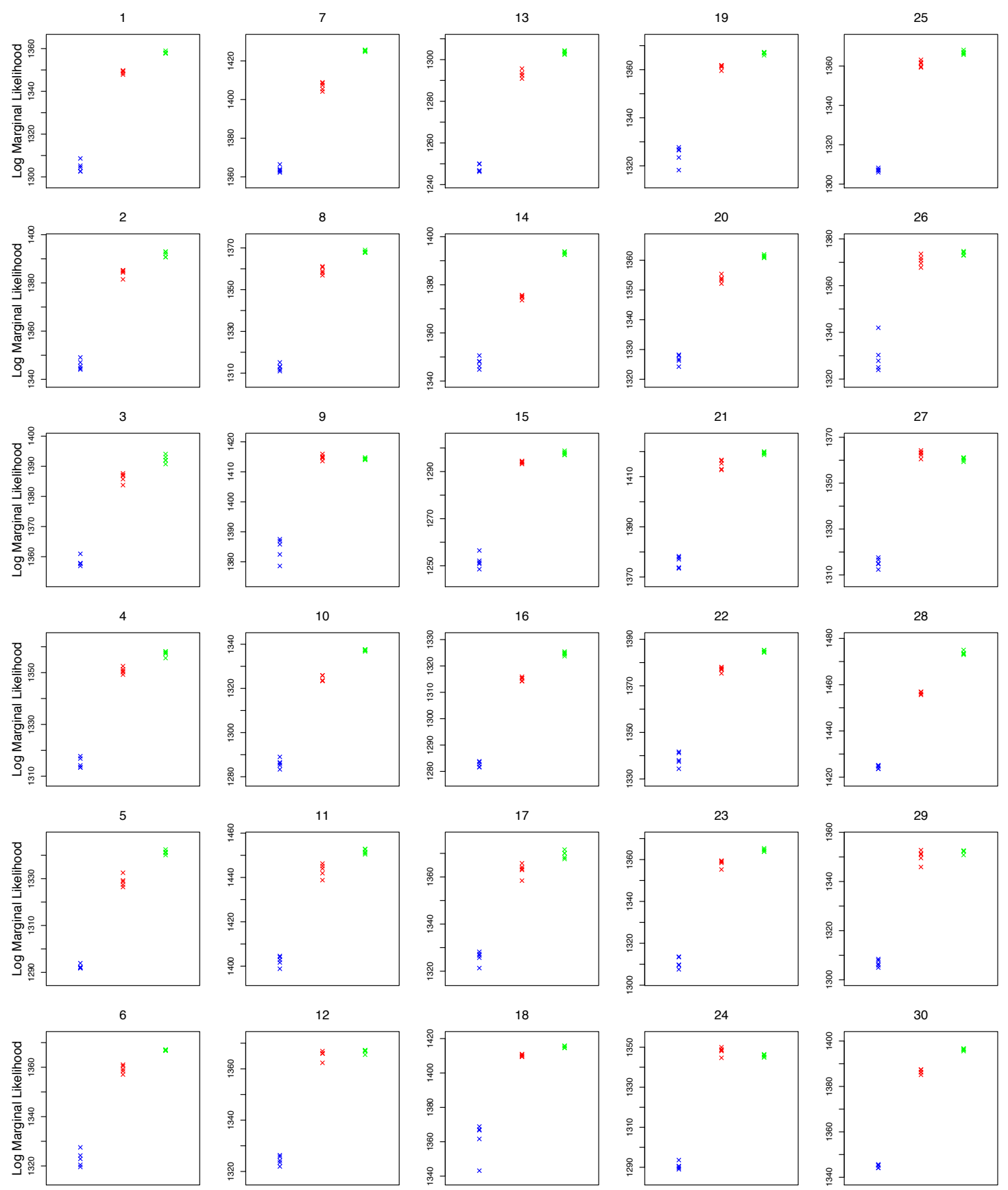

Figure 14. Bayes factor analysis for the recovery analysis. These data are simulated from SSP, based on the data from Servant et al. (2015). 

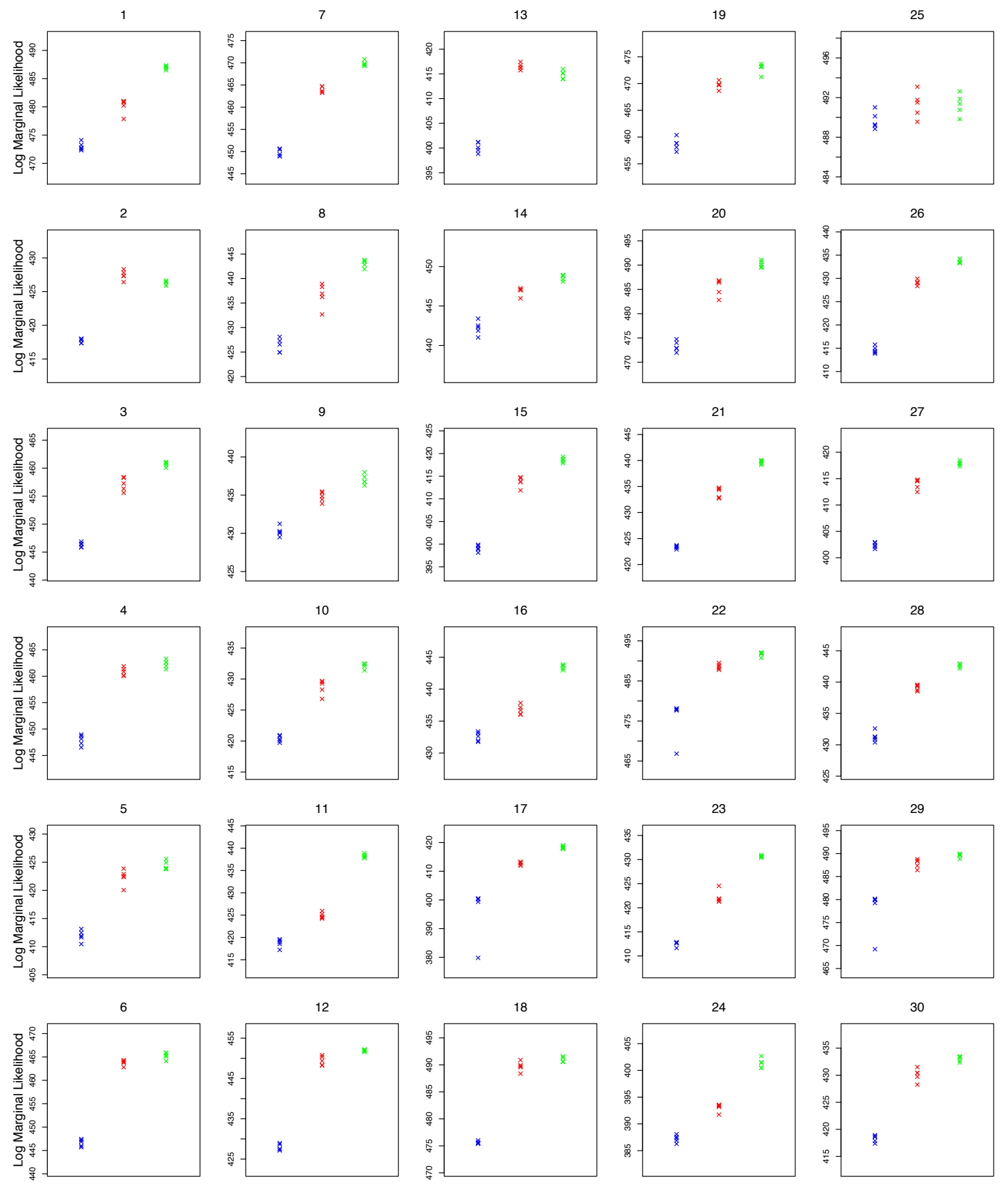

Figure 15. Bayes factor analysis for the recovery analysis. These data are simulated from SSP, based on the data from Ulrich et al. (2015). 

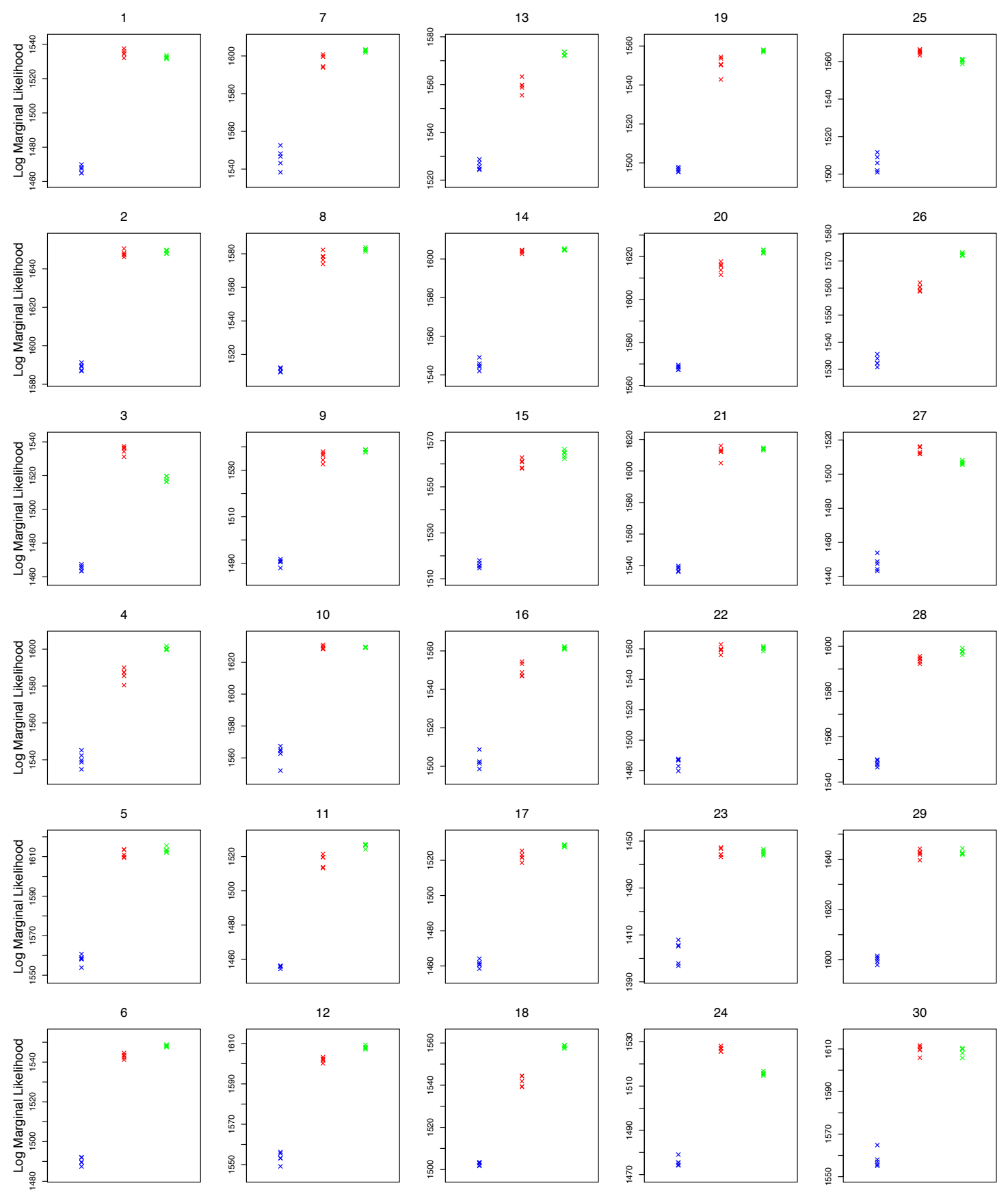

Figure 16. Bayes factor analysis for the recovery analysis. These data are simulated from DSTP, based on the data from Servant et al. (2015). 

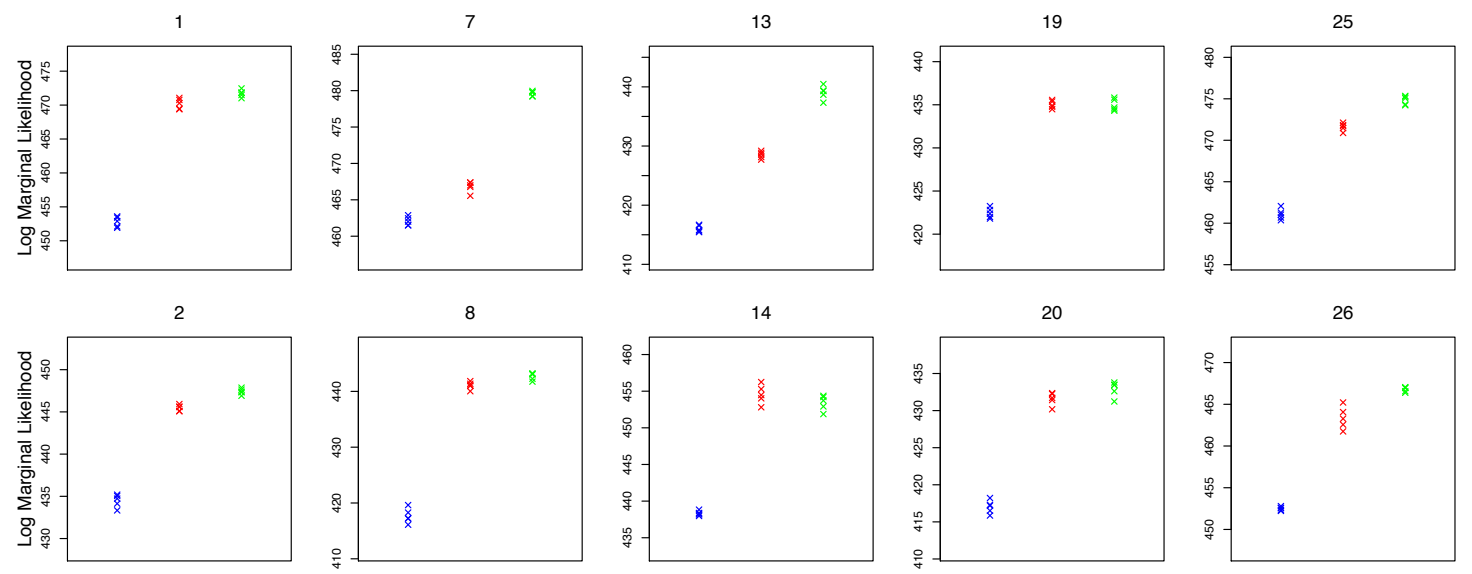

14
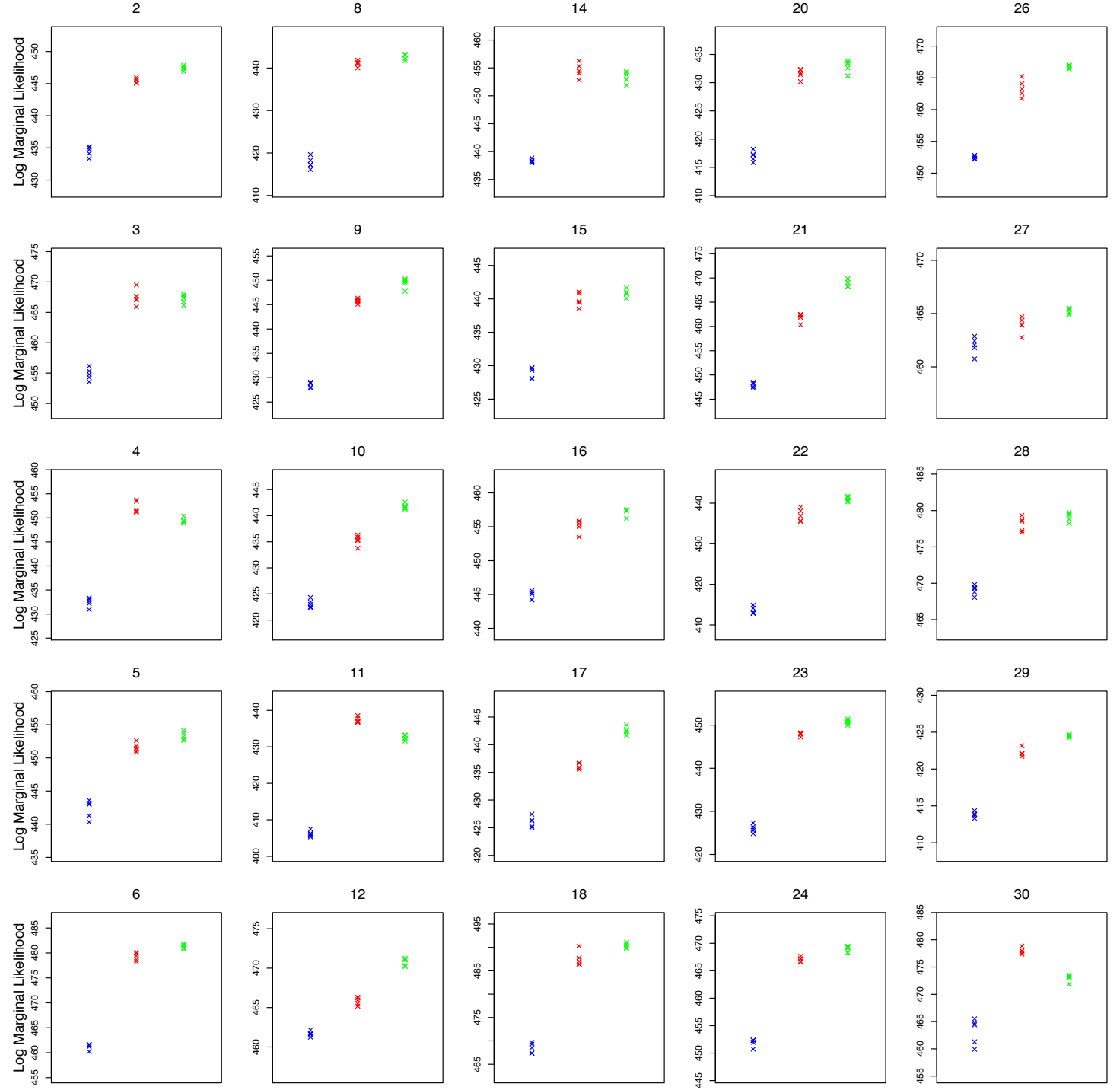

Figure 17. Bayes factor analysis for the recovery analysis. These data are simulated from DSTP, based on the data from Ulrich et al. (2015). 
Parameter estimates for the models

The tables below display the estimated parameters for each participant from each model in each data set. Specifically, for brevity, we display the posterior means, which reflect the expected value over the posterior distribution.

Table 19: Mean posterior estimates from the simple DMC model in the White et al. (2011) data set.

\begin{tabular}{lrrrrrrr}
\hline Subject & $\mathrm{v}_{c}$ & $\mathrm{a}$ & ter & $\mathrm{z}$ & $\zeta$ & $\alpha$ & $\tau$ \\
\hline 1 & 0.31 & 0.06 & 0.36 & 0.50 & 0.33 & 1.37 & 524.27 \\
2 & 0.24 & 0.05 & 0.33 & 0.49 & 44.03 & 11.04 & 259.38 \\
3 & 0.35 & 0.06 & 0.33 & 0.49 & 4.84 & 1.79 & 521.08 \\
4 & 0.29 & 0.05 & 0.30 & 0.50 & 0.96 & 1.46 & 669.51 \\
5 & 0.33 & 0.06 & 0.31 & 0.46 & 5.08 & 1.81 & 416.81 \\
6 & 0.18 & 0.05 & 0.29 & 0.49 & 5.15 & 1.80 & 532.71 \\
7 & 0.35 & 0.06 & 0.33 & 0.49 & 0.82 & 1.56 & 483.49 \\
8 & 0.25 & 0.06 & 0.29 & 0.45 & 80.86 & 10.39 & 507.08 \\
9 & 0.29 & 0.07 & 0.30 & 0.51 & 45.12 & 8.66 & 527.55 \\
10 & 0.25 & 0.05 & 0.30 & 0.49 & 7.14 & 1.88 & 571.20 \\
11 & 0.31 & 0.05 & 0.30 & 0.53 & 0.14 & 1.22 & 432.82 \\
12 & 0.31 & 0.06 & 0.28 & 0.47 & 36.11 & 2.13 & 523.03 \\
13 & 0.25 & 0.05 & 0.29 & 0.52 & 53.93 & 8.46 & 495.64 \\
14 & 0.31 & 0.06 & 0.33 & 0.45 & 48.55 & 2.64 & 357.21 \\
15 & 0.22 & 0.04 & 0.28 & 0.48 & 0.69 & 1.39 & 596.84 \\
16 & 0.31 & 0.04 & 0.29 & 0.52 & 3.21 & 1.47 & 677.35 \\
17 & 0.20 & 0.05 & 0.26 & 0.49 & 35.36 & 2.08 & 529.14 \\
18 & 0.19 & 0.05 & 0.31 & 0.47 & 3.90 & 1.64 & 549.05 \\
19 & 0.28 & 0.06 & 0.29 & 0.51 & 58.83 & 2.12 & 518.86 \\
20 & 0.39 & 0.06 & 0.33 & 0.52 & 87.03 & 2.28 & 576.90 \\
21 & 0.32 & 0.06 & 0.33 & 0.51 & 35.12 & 1.99 & 552.40 \\
22 & 0.32 & 0.06 & 0.32 & 0.51 & 0.70 & 1.45 & 285.92 \\
23 & 0.30 & 0.05 & 0.33 & 0.53 & 0.14 & 1.23 & 455.29 \\
24 & 0.27 & 0.06 & 0.30 & 0.53 & 1.27 & 1.48 & 534.58 \\
25 & 0.30 & 0.05 & 0.32 & 0.53 & 0.47 & 1.34 & 630.71 \\
$\mu$ & 0.28 & 0.05 & 0.31 & 0.50 & 12.71 & 1.88 & 495.54 \\
\hline & & & & & & &
\end{tabular}


Table 20: Mean posterior estimates from the simple DMC model in the Ulrich et al. (2015) data set.

\begin{tabular}{lrrrrrrr}
\hline Subject & $\mathrm{v}_{c}$ & $\mathrm{a}$ & ter & $\mathrm{z}$ & $\zeta$ & $\alpha$ & $\tau$ \\
\hline 1 & 0.38 & 0.05 & 0.30 & 0.54 & 32.40 & 7.56 & 181.76 \\
2 & 0.35 & 0.04 & 0.31 & 0.44 & 17.49 & 2.00 & 188.79 \\
3 & 0.27 & 0.04 & 0.27 & 0.52 & 29.23 & 5.81 & 182.43 \\
4 & 0.30 & 0.05 & 0.32 & 0.46 & 25.14 & 6.27 & 180.00 \\
5 & 0.31 & 0.04 & 0.27 & 0.49 & 26.47 & 5.77 & 179.75 \\
6 & 0.31 & 0.04 & 0.26 & 0.43 & 23.26 & 2.13 & 184.32 \\
7 & 0.28 & 0.04 & 0.24 & 0.46 & 23.51 & 2.23 & 187.81 \\
8 & 0.35 & 0.04 & 0.30 & 0.49 & 32.13 & 8.35 & 179.79 \\
9 & 0.32 & 0.04 & 0.24 & 0.50 & 29.56 & 5.78 & 180.48 \\
10 & 0.39 & 0.04 & 0.31 & 0.52 & 5.53 & 1.00 & 142.66 \\
11 & 0.28 & 0.05 & 0.28 & 0.41 & 30.61 & 7.23 & 190.38 \\
12 & 0.29 & 0.04 & 0.31 & 0.51 & 25.62 & 6.32 & 182.17 \\
13 & 0.49 & 0.05 & 0.31 & 0.50 & 15.80 & 2.01 & 194.30 \\
14 & 0.43 & 0.05 & 0.27 & 0.52 & 21.69 & 7.38 & 193.03 \\
15 & 0.42 & 0.04 & 0.29 & 0.53 & 26.41 & 2.23 & 175.30 \\
16 & 0.37 & 0.05 & 0.28 & 0.47 & 22.18 & 2.24 & 182.87 \\
$\mu$ & 0.35 & 0.04 & 0.29 & 0.49 & 22.36 & 2.90 & 181.10 \\
\hline
\end{tabular}

Table 21: Mean posterior estimates from the simple DMC model in the Servant et al. (2015) data set.

\begin{tabular}{lrrrrrrr}
\hline Subject & $\mathrm{v}_{c}$ & $\mathrm{a}$ & ter & $\mathrm{z}$ & $\zeta$ & $\alpha$ & $\tau$ \\
\hline 1 & 0.42 & 0.04 & 0.27 & 0.50 & 15.98 & 2.00 & 306.01 \\
2 & 0.30 & 0.06 & 0.27 & 0.49 & 48.40 & 10.53 & 273.36 \\
3 & 0.28 & 0.06 & 0.27 & 0.47 & 47.32 & 17.24 & 271.01 \\
4 & 0.24 & 0.05 & 0.27 & 0.47 & 24.56 & 2.26 & 242.45 \\
5 & 0.30 & 0.05 & 0.21 & 0.49 & 45.38 & 5.22 & 276.09 \\
6 & 0.40 & 0.05 & 0.23 & 0.54 & 10.50 & 2.02 & 290.09 \\
7 & 0.29 & 0.07 & 0.28 & 0.47 & 32.92 & 18.70 & 279.69 \\
8 & 0.36 & 0.05 & 0.25 & 0.46 & 60.78 & 2.26 & 287.88 \\
9 & 0.31 & 0.06 & 0.25 & 0.42 & 54.39 & 13.89 & 283.45 \\
10 & 0.30 & 0.06 & 0.26 & 0.49 & 38.82 & 13.02 & 268.91 \\
11 & 0.28 & 0.05 & 0.26 & 0.54 & 0.84 & 1.63 & 265.40 \\
12 & 0.32 & 0.06 & 0.26 & 0.56 & 60.22 & 27.41 & 256.89 \\
$\mu$ & 0.32 & 0.05 & 0.26 & 0.49 & 34.20 & 4.43 & 272.72 \\
\hline
\end{tabular}


Table 22: Mean posterior estimates from the complex DMC model in the White et al. (2011) data set.

\begin{tabular}{lrrrrrrrrrr}
\hline Subject & $\mathrm{v}_{c}$ & $\mathrm{a}$ & ter & $\mathrm{z}$ & $\zeta$ & $\alpha$ & $\tau$ & $\mathrm{s}_{\text {ter }}$ & $\mathrm{s}_{v}$ & $\mathrm{~s}_{z}$ \\
\hline 1 & 1.23 & 0.11 & 0.42 & 0.50 & 0.68 & 1.32 & 313.04 & 0.15 & 0.39 & 0.20 \\
2 & 0.44 & 0.06 & 0.37 & 0.49 & 0.73 & 1.49 & 331.26 & 0.14 & 0.14 & 0.06 \\
3 & 0.59 & 0.06 & 0.37 & 0.48 & 1.27 & 1.51 & 344.09 & 0.15 & 0.09 & 0.10 \\
4 & 0.50 & 0.06 & 0.35 & 0.50 & 2.15 & 1.70 & 174.42 & 0.18 & 0.09 & 0.11 \\
5 & 0.72 & 0.07 & 0.36 & 0.45 & 0.62 & 1.38 & 425.53 & 0.12 & 0.21 & 0.10 \\
6 & 0.64 & 0.07 & 0.41 & 0.51 & 1.27 & 1.44 & 286.37 & 0.28 & 0.24 & 0.17 \\
7 & 0.95 & 0.09 & 0.38 & 0.47 & 2.03 & 1.55 & 385.76 & 0.12 & 0.28 & 0.15 \\
8 & 0.88 & 0.11 & 0.36 & 0.47 & 1.18 & 1.44 & 328.23 & 0.17 & 0.29 & 0.22 \\
9 & 0.37 & 0.06 & 0.38 & 0.55 & 0.22 & 1.15 & 362.11 & 0.25 & 0.10 & 0.04 \\
10 & 0.76 & 0.08 & 0.35 & 0.50 & 0.38 & 1.12 & 219.17 & 0.13 & 0.25 & 0.17 \\
11 & 1.28 & 0.12 & 0.36 & 0.52 & 1.68 & 1.02 & 258.60 & 0.18 & 0.36 & 0.26 \\
12 & 0.79 & 0.07 & 0.35 & 0.46 & 1.19 & 1.53 & 303.42 & 0.19 & 0.19 & 0.15 \\
13 & 0.53 & 0.06 & 0.37 & 0.54 & 0.35 & 1.37 & 384.66 & 0.23 & 0.12 & 0.12 \\
14 & 1.22 & 0.11 & 0.42 & 0.47 & 27.97 & 1.00 & 295.73 & 0.14 & 0.43 & 0.24 \\
15 & 0.55 & 0.05 & 0.32 & 0.47 & 0.76 & 1.38 & 303.55 & 0.16 & 0.27 & 0.10 \\
16 & 0.93 & 0.07 & 0.33 & 0.52 & 1.21 & 1.43 & 384.07 & 0.13 & 0.39 & 0.13 \\
17 & 0.66 & 0.07 & 0.36 & 0.51 & 1.80 & 1.01 & 270.89 & 0.30 & 0.24 & 0.17 \\
18 & 0.46 & 0.07 & 0.38 & 0.48 & 0.25 & 1.12 & 331.94 & 0.17 & 0.17 & 0.15 \\
19 & 0.64 & 0.09 & 0.35 & 0.51 & 0.58 & 1.39 & 244.68 & 0.15 & 0.18 & 0.14 \\
20 & 0.91 & 0.07 & 0.41 & 0.54 & 5.59 & 1.72 & 403.40 & 0.18 & 0.17 & 0.12 \\
21 & 1.55 & 0.12 & 0.41 & 0.50 & 0.68 & 1.15 & 175.60 & 0.19 & 0.49 & 0.25 \\
22 & 1.81 & 0.17 & 0.38 & 0.50 & 14.67 & 1.00 & 164.39 & 0.15 & 0.50 & 0.35 \\
23 & 0.65 & 0.07 & 0.38 & 0.52 & 0.41 & 1.34 & 357.08 & 0.15 & 0.16 & 0.12 \\
24 & 1.38 & 0.16 & 0.38 & 0.52 & 0.31 & 1.13 & 280.09 & 0.21 & 0.36 & 0.36 \\
25 & 2.32 & 0.18 & 0.38 & 0.48 & 1.90 & 1.30 & 330.78 & 0.17 & 0.74 & 0.44 \\
$\mu$ & 0.84 & 0.09 & 0.37 & 0.50 & 0.65 & 1.15 & 303.21 & 0.17 & 0.18 & 0.13 \\
\hline & & & & & & & & & &
\end{tabular}


Table 23: Mean posterior estimates from the complex DMC model in the Ulrich et al. (2015) data set.

\begin{tabular}{lrrrrrrrrrr}
\hline Subject & $\mathrm{v}_{c}$ & $\mathrm{a}$ & ter & $\mathrm{z}$ & $\zeta$ & $\alpha$ & $\tau$ & $\mathrm{s}_{\text {ter }}$ & $\mathrm{s}_{v}$ & $\mathrm{~s}_{z}$ \\
\hline 1 & 0.90 & 0.06 & 0.35 & 0.54 & 15.07 & 3.65 & 195.70 & 0.17 & 0.26 & 0.10 \\
2 & 0.99 & 0.05 & 0.35 & 0.45 & 3.55 & 1.69 & 195.93 & 0.12 & 0.34 & 0.10 \\
3 & 0.63 & 0.05 & 0.33 & 0.52 & 14.22 & 3.75 & 195.88 & 0.16 & 0.15 & 0.13 \\
4 & 0.58 & 0.06 & 0.37 & 0.46 & 16.47 & 5.17 & 196.79 & 0.16 & 0.17 & 0.10 \\
5 & 0.57 & 0.04 & 0.32 & 0.47 & 13.88 & 3.49 & 193.23 & 0.16 & 0.13 & 0.08 \\
6 & 0.45 & 0.04 & 0.28 & 0.45 & 13.01 & 2.03 & 197.67 & 0.19 & 0.08 & 0.06 \\
7 & 0.72 & 0.05 & 0.30 & 0.45 & 8.94 & 1.88 & 196.58 & 0.26 & 0.12 & 0.13 \\
8 & 1.17 & 0.06 & 0.35 & 0.49 & 14.29 & 1.84 & 196.11 & 0.14 & 0.46 & 0.13 \\
9 & 1.27 & 0.06 & 0.32 & 0.53 & 11.47 & 2.16 & 194.38 & 0.25 & 0.27 & 0.15 \\
10 & 0.98 & 0.07 & 0.33 & 0.53 & 0.22 & 1.17 & 204.27 & 0.07 & 0.18 & 0.13 \\
11 & 0.60 & 0.06 & 0.34 & 0.41 & 14.60 & 2.38 & 196.39 & 0.20 & 0.17 & 0.10 \\
12 & 0.77 & 0.05 & 0.38 & 0.53 & 13.56 & 4.00 & 196.38 & 0.20 & 0.26 & 0.10 \\
13 & 1.02 & 0.07 & 0.35 & 0.50 & 4.96 & 1.74 & 190.02 & 0.10 & 0.22 & 0.10 \\
14 & 1.23 & 0.06 & 0.33 & 0.53 & 20.11 & 2.36 & 190.51 & 0.17 & 0.30 & 0.11 \\
15 & 0.99 & 0.06 & 0.34 & 0.52 & 16.57 & 2.02 & 199.05 & 0.13 & 0.20 & 0.11 \\
16 & 0.64 & 0.06 & 0.31 & 0.47 & 13.27 & 2.03 & 196.57 & 0.13 & 0.16 & 0.08 \\
$\mu$ & 0.80 & 0.06 & 0.33 & 0.49 & 10.19 & 1.88 & 195.85 & 0.16 & 0.16 & 0.11 \\
\hline
\end{tabular}

Table 24: Mean posterior estimates from the complex DMC model in the Servant et al. (2015) data set.

\begin{tabular}{lrrrrrrrrrr}
\hline Subject & $\mathrm{v}_{c}$ & $\mathrm{a}$ & ter & $\mathrm{z}$ & $\zeta$ & $\alpha$ & $\tau$ & $\mathrm{s}_{\text {ter }}$ & $\mathrm{s}_{v}$ & $\mathrm{~s}_{z}$ \\
\hline 1 & 0.55 & 0.03 & 0.32 & 0.50 & 12.65 & 1.94 & 270.69 & 0.16 & 0.05 & 0.04 \\
2 & 0.46 & 0.05 & 0.33 & 0.49 & 0.42 & 1.59 & 265.76 & 0.21 & 0.09 & 0.08 \\
3 & 0.35 & 0.06 & 0.31 & 0.47 & 36.68 & 4.91 & 259.54 & 0.18 & 0.03 & 0.08 \\
4 & 0.33 & 0.05 & 0.32 & 0.47 & 4.99 & 1.91 & 268.51 & 0.22 & 0.10 & 0.05 \\
5 & 0.40 & 0.04 & 0.25 & 0.49 & 23.89 & 7.44 & 268.54 & 0.23 & 0.07 & 0.06 \\
6 & 0.53 & 0.05 & 0.26 & 0.55 & 3.17 & 1.81 & 263.72 & 0.16 & 0.10 & 0.03 \\
7 & 0.57 & 0.07 & 0.34 & 0.46 & 0.24 & 1.43 & 273.17 & 0.20 & 0.16 & 0.11 \\
8 & 0.97 & 0.08 & 0.30 & 0.48 & 39.24 & 2.03 & 284.70 & 0.17 & 0.25 & 0.18 \\
9 & 0.49 & 0.07 & 0.31 & 0.43 & 23.83 & 2.08 & 239.94 & 0.21 & 0.07 & 0.10 \\
10 & 0.39 & 0.06 & 0.31 & 0.50 & 42.92 & 6.93 & 271.08 & 0.19 & 0.07 & 0.07 \\
11 & 0.40 & 0.05 & 0.29 & 0.54 & 2.41 & 1.76 & 253.26 & 0.16 & 0.09 & 0.07 \\
12 & 0.67 & 0.08 & 0.33 & 0.56 & 1.10 & 1.52 & 266.23 & 0.20 & 0.18 & 0.12 \\
$\mu$ & 0.50 & 0.06 & 0.31 & 0.49 & 11.51 & 2.05 & 264.63 & 0.19 & 0.07 & 0.06 \\
\hline
\end{tabular}


Table 25: Mean posterior estimates from the simple DSTP model in the White et al. (2011) data set.

\begin{tabular}{lrrrrrrrr}
\hline Subject & $\mathrm{v}_{t a}$ & $\mathrm{a}$ & ter & $\mathrm{z}$ & $\mathrm{a}_{s s}$ & $\mathrm{v}_{f l}$ & $\mathrm{v}_{s s}$ & $\mathrm{v}_{p 2}$ \\
\hline 1 & 0.10 & 0.06 & 0.34 & 0.50 & 0.41 & 0.44 & 12.56 & 0.38 \\
2 & 0.28 & 0.05 & 0.34 & 0.48 & 0.90 & 0.27 & 15.57 & 0.25 \\
3 & 0.17 & 0.06 & 0.31 & 0.49 & 0.15 & 0.19 & 1.58 & 0.46 \\
4 & 0.09 & 0.06 & 0.28 & 0.50 & 0.40 & 0.27 & 6.00 & 0.37 \\
5 & 0.33 & 0.06 & 0.31 & 0.46 & 0.52 & 0.34 & 11.50 & 0.34 \\
6 & 0.04 & 0.07 & 0.24 & 0.49 & 0.10 & 0.08 & 0.32 & 1.22 \\
7 & 0.17 & 0.06 & 0.32 & 0.47 & 0.80 & 0.36 & 14.37 & 0.40 \\
8 & 0.06 & 0.06 & 0.28 & 0.45 & 0.74 & 0.31 & 17.44 & 0.32 \\
9 & -0.02 & 0.08 & 0.29 & 0.52 & 0.57 & 0.35 & 12.49 & 0.35 \\
10 & 0.09 & 0.05 & 0.29 & 0.49 & 0.22 & 0.31 & 4.78 & 0.33 \\
11 & 0.13 & 0.07 & 0.26 & 0.53 & 0.15 & 0.16 & 1.05 & 0.88 \\
12 & 0.11 & 0.08 & 0.24 & 0.46 & 0.08 & 0.11 & 0.40 & 1.30 \\
13 & 0.06 & 0.07 & 0.23 & 0.52 & 0.09 & 0.10 & 0.42 & 0.95 \\
14 & 0.11 & 0.07 & 0.33 & 0.44 & 0.38 & 0.72 & 14.52 & 0.36 \\
15 & 0.13 & 0.04 & 0.27 & 0.48 & 0.74 & 0.23 & 7.44 & 0.32 \\
16 & 0.12 & 0.05 & 0.28 & 0.52 & 0.59 & 0.47 & 15.02 & 0.36 \\
17 & 0.03 & 0.07 & 0.20 & 0.50 & 0.09 & 0.08 & 0.30 & 1.38 \\
18 & 0.08 & 0.05 & 0.30 & 0.47 & 1.04 & 0.19 & 7.66 & 0.28 \\
19 & 0.07 & 0.06 & 0.29 & 0.51 & 0.43 & 0.42 & 12.76 & 0.32 \\
20 & 0.15 & 0.08 & 0.28 & 0.52 & 0.12 & 0.15 & 0.60 & 1.56 \\
21 & 0.03 & 0.06 & 0.32 & 0.50 & 0.37 & 0.41 & 10.34 & 0.42 \\
22 & 0.10 & 0.06 & 0.31 & 0.51 & 0.71 & 0.44 & 16.45 & 0.39 \\
23 & 0.04 & 0.06 & 0.31 & 0.52 & 0.38 & 0.37 & 9.92 & 0.38 \\
24 & 0.10 & 0.07 & 0.27 & 0.52 & 0.54 & 0.16 & 4.68 & 0.39 \\
25 & 0.15 & 0.05 & 0.30 & 0.53 & 1.01 & 0.26 & 11.17 & 0.41 \\
$\mu$ & 0.11 & 0.06 & 0.29 & 0.50 & 0.15 & 0.28 & 1.03 & 0.57 \\
\hline & & & & & & & &
\end{tabular}


Table 26: Mean posterior estimates from the simple DSTP model in the Ulrich et al. (2015) data set.

\begin{tabular}{lrrrrrrrr}
\hline Subject & $\mathrm{v}_{t a}$ & $\mathrm{a}$ & ter & $\mathrm{z}$ & $\mathrm{a}_{s s}$ & $\mathrm{v}_{f l}$ & $\mathrm{v}_{s s}$ & $\mathrm{v}_{p 2}$ \\
\hline 1 & 0.14 & 0.07 & 0.25 & 0.55 & 0.09 & 0.10 & 0.55 & 2.85 \\
2 & 0.32 & 0.04 & 0.30 & 0.48 & 0.08 & 0.13 & 0.29 & 3.51 \\
3 & -0.04 & 0.07 & 0.22 & 0.52 & 0.07 & 0.09 & 0.47 & 1.69 \\
4 & 0.18 & 0.06 & 0.29 & 0.47 & 0.08 & 0.04 & 0.29 & 3.08 \\
5 & 0.03 & 0.07 & 0.22 & 0.49 & 0.09 & 0.07 & 0.52 & 2.93 \\
6 & 0.06 & 0.06 & 0.20 & 0.46 & 0.09 & 0.11 & 0.50 & 2.58 \\
7 & -0.02 & 0.07 & 0.16 & 0.46 & 0.11 & 0.08 & 0.61 & 1.61 \\
8 & 0.12 & 0.07 & 0.27 & 0.49 & 0.07 & 0.13 & 0.38 & 3.81 \\
9 & 0.06 & 0.07 & 0.19 & 0.50 & 0.11 & 0.11 & 0.66 & 2.01 \\
10 & 0.21 & 0.06 & 0.28 & 0.53 & 0.06 & 0.20 & 0.73 & 0.72 \\
11 & 0.06 & 0.08 & 0.22 & 0.41 & 0.09 & 0.08 & 0.38 & 2.69 \\
12 & -0.03 & 0.08 & 0.24 & 0.51 & 0.08 & 0.12 & 0.46 & 1.94 \\
13 & 0.29 & 0.07 & 0.29 & 0.51 & 0.07 & 0.22 & 0.45 & 2.68 \\
14 & 0.08 & 0.07 & 0.23 & 0.52 & 0.07 & 0.12 & 0.66 & 1.49 \\
15 & 0.16 & 0.06 & 0.26 & 0.54 & 0.08 & 0.14 & 0.57 & 2.40 \\
16 & 0.36 & 0.05 & 0.28 & 0.46 & 0.07 & 0.10 & 0.55 & 0.42 \\
$\mu$ & 0.12 & 0.07 & 0.24 & 0.49 & 0.08 & 0.12 & 0.50 & 2.09 \\
\hline
\end{tabular}

Table 27: Mean posterior estimates from the simple DSTP model in the Servant et al. (2015) data set.

\begin{tabular}{lrrrrrrrr}
\hline Subject & $\mathrm{v}_{t a}$ & $\mathrm{a}$ & ter & $\mathrm{z}$ & $\mathrm{a}_{s s}$ & $\mathrm{v}_{f l}$ & $\mathrm{v}_{s s}$ & $\mathrm{v}_{p 2}$ \\
\hline 1 & 0.16 & 0.05 & 0.24 & 0.51 & 0.09 & 0.14 & 0.65 & 2.12 \\
2 & 0.13 & 0.07 & 0.23 & 0.49 & 0.08 & 0.06 & 0.36 & 1.38 \\
3 & 0.10 & 0.08 & 0.21 & 0.47 & 0.09 & 0.04 & 0.33 & 1.86 \\
4 & 0.10 & 0.07 & 0.23 & 0.47 & 0.08 & 0.07 & 0.27 & 2.00 \\
5 & 0.04 & 0.08 & 0.14 & 0.48 & 0.08 & 0.06 & 0.42 & 1.65 \\
6 & 0.22 & 0.06 & 0.21 & 0.56 & 0.07 & 0.12 & 0.38 & 2.29 \\
7 & 0.11 & 0.10 & 0.22 & 0.46 & 0.08 & 0.05 & 0.27 & 1.64 \\
8 & 0.13 & 0.05 & 0.23 & 0.48 & 0.09 & 0.18 & 1.45 & 0.40 \\
9 & 0.12 & 0.08 & 0.20 & 0.42 & 0.09 & 0.08 & 0.42 & 1.03 \\
10 & 0.13 & 0.08 & 0.21 & 0.49 & 0.09 & 0.05 & 0.30 & 2.59 \\
11 & 0.19 & 0.05 & 0.25 & 0.54 & 0.08 & 0.13 & 1.05 & 0.34 \\
12 & 0.10 & 0.09 & 0.21 & 0.57 & 0.08 & 0.07 & 0.35 & 1.28 \\
$\mu$ & 0.13 & 0.07 & 0.21 & 0.49 & 0.08 & 0.09 & 0.50 & 1.49 \\
\hline
\end{tabular}


Table 28: Mean posterior estimates from the complex DSTP model in the White et al. (2011) data set.

\begin{tabular}{lrrrrrrrrrrr}
\hline Subject & $\mathrm{v}_{t a}$ & $\mathrm{a}$ & $\mathrm{ter}$ & $\mathrm{z}$ & $\mathrm{a}_{s s}$ & $\mathrm{v}_{f l}$ & $\mathrm{v}_{s s}$ & $\mathrm{v}_{p 2}$ & $\mathrm{~s}_{t e r}$ & $\mathrm{~s}_{v}$ & $\mathrm{~s}_{z}$ \\
\hline 1 & 0.76 & 1.56 & 0.25 & 0.50 & 1.02 & 3.00 & 7.83 & 11.09 & 0.09 & 2.86 & 0.15 \\
2 & 0.51 & 1.69 & 0.26 & 0.48 & 0.85 & 4.21 & 7.77 & 12.53 & 0.06 & 3.94 & 0.28 \\
3 & 0.36 & 1.39 & 0.23 & 0.49 & 1.33 & 2.54 & 9.63 & 11.65 & 0.11 & 2.95 & 0.29 \\
4 & 0.17 & 1.26 & 0.23 & 0.50 & 1.13 & 3.56 & 8.92 & 10.77 & 0.14 & 3.28 & 0.21 \\
5 & 0.02 & 1.28 & 0.26 & 0.44 & 0.52 & 5.85 & 7.81 & 9.70 & 0.11 & 3.24 & 0.24 \\
6 & 0.26 & 1.02 & 0.21 & 0.47 & 1.43 & 1.69 & 5.68 & 10.54 & 0.17 & 2.24 & 0.20 \\
7 & 0.57 & 0.19 & 0.33 & 0.45 & 0.35 & 0.96 & 4.49 & 1.73 & 0.11 & 0.68 & 0.07 \\
8 & 0.19 & 1.56 & 0.20 & 0.46 & 1.02 & 3.27 & 7.34 & 10.35 & 0.09 & 3.13 & 0.27 \\
9 & -0.41 & 1.40 & 0.18 & 0.51 & 0.90 & 2.06 & 6.56 & 7.09 & 0.13 & 2.11 & 0.17 \\
10 & -0.05 & 1.23 & 0.23 & 0.48 & 1.05 & 3.36 & 7.51 & 13.30 & 0.13 & 3.13 & 0.19 \\
11 & 0.58 & 1.26 & 0.21 & 0.53 & 1.44 & 2.80 & 9.65 & 10.89 & 0.09 & 2.76 & 0.36 \\
12 & -0.50 & 1.02 & 0.22 & 0.45 & 0.88 & 1.88 & 7.78 & 9.10 & 0.19 & 2.52 & 0.20 \\
13 & -0.01 & 1.21 & 0.19 & 0.52 & 1.31 & 1.73 & 7.69 & 10.55 & 0.14 & 2.67 & 0.22 \\
14 & 0.30 & 1.65 & 0.27 & 0.45 & 1.35 & 5.58 & 14.38 & 10.79 & 0.08 & 3.73 & 0.26 \\
15 & 1.41 & 0.56 & 0.26 & 0.47 & 0.99 & 3.06 & 6.94 & 9.30 & 0.12 & 3.10 & 0.22 \\
16 & 0.82 & 0.83 & 0.24 & 0.52 & 0.70 & 4.28 & 7.39 & 9.20 & 0.04 & 3.16 & 0.19 \\
17 & 0.11 & 1.22 & 0.21 & 0.49 & 1.51 & 2.14 & 8.46 & 13.00 & 0.29 & 3.60 & 0.35 \\
18 & 0.55 & 1.29 & 0.27 & 0.46 & 1.46 & 4.23 & 8.78 & 11.79 & 0.18 & 4.13 & 0.25 \\
19 & -0.06 & 1.43 & 0.22 & 0.51 & 1.01 & 3.31 & 9.63 & 9.08 & 0.06 & 3.25 & 0.27 \\
20 & 0.25 & 0.96 & 0.25 & 0.51 & 1.08 & 1.86 & 7.44 & 8.89 & 0.07 & 1.87 & 0.17 \\
21 & -0.07 & 1.49 & 0.23 & 0.50 & 1.30 & 3.01 & 9.09 & 12.19 & 0.12 & 2.89 & 0.32 \\
22 & -0.09 & 2.27 & 0.22 & 0.51 & 1.50 & 6.52 & 11.50 & 18.10 & 0.08 & 4.27 & 0.32 \\
23 & 0.66 & 1.29 & 0.25 & 0.53 & 1.17 & 2.96 & 9.65 & 10.28 & 0.08 & 3.26 & 0.25 \\
24 & 0.12 & 1.49 & 0.22 & 0.52 & 1.67 & 3.03 & 11.40 & 10.13 & 0.12 & 3.17 & 0.31 \\
25 & 1.11 & 0.75 & 0.27 & 0.52 & 0.95 & 2.57 & 6.72 & 8.87 & 0.10 & 2.36 & 0.20 \\
$\mu$ & 0.29 & 0.50 & 0.24 & 0.49 & 0.61 & 0.54 & 1.04 & 6.33 & 0.08 & 0.32 & 0.15 \\
\hline & & & & & & & & & & &
\end{tabular}


Table 29: Mean posterior estimates from the complex DSTP model in the Ulrich et al. (2015) data set.

\begin{tabular}{lrrrrrrrrrrr}
\hline Subject & $\mathrm{v}_{t a}$ & $\mathrm{a}$ & ter & $\mathrm{z}$ & $\mathrm{a}_{s s}$ & $\mathrm{v}_{f l}$ & $\mathrm{v}_{s s}$ & $\mathrm{v}_{p 2}$ & $\mathrm{~s}_{t e r}$ & $\mathrm{~s}_{v}$ & $\mathrm{~s}_{z}$ \\
\hline 1 & 0.16 & 0.38 & 0.24 & 0.55 & 0.29 & 0.79 & 2.68 & 5.22 & 0.15 & 0.92 & 0.15 \\
2 & 0.25 & 0.33 & 0.26 & 0.45 & 0.23 & 1.34 & 2.53 & 4.87 & 0.06 & 1.04 & 0.20 \\
3 & -0.28 & 0.31 & 0.22 & 0.53 & 0.31 & 0.48 & 2.65 & 4.76 & 0.14 & 1.03 & 0.22 \\
4 & 0.08 & 0.41 & 0.25 & 0.45 & 0.32 & 0.38 & 2.47 & 4.04 & 0.13 & 0.98 & 0.18 \\
5 & 0.05 & 0.30 & 0.22 & 0.48 & 0.33 & 0.25 & 2.69 & 4.82 & 0.13 & 0.96 & 0.17 \\
6 & 0.09 & 0.27 & 0.20 & 0.45 & 0.34 & 0.58 & 2.76 & 5.27 & 0.14 & 1.04 & 0.20 \\
7 & -0.29 & 0.28 & 0.20 & 0.45 & 0.33 & 0.46 & 2.91 & 4.35 & 0.23 & 0.92 & 0.24 \\
8 & 0.37 & 0.31 & 0.26 & 0.48 & 0.31 & 0.93 & 2.66 & 5.54 & 0.09 & 1.01 & 0.14 \\
9 & -0.15 & 0.27 & 0.21 & 0.48 & 0.32 & 0.50 & 3.15 & 4.42 & 0.21 & 0.89 & 0.22 \\
10 & -0.03 & 0.40 & 0.22 & 0.52 & 0.27 & 1.03 & 2.78 & 4.23 & 0.06 & 0.80 & 0.18 \\
11 & -0.31 & 0.42 & 0.22 & 0.42 & 0.31 & 0.58 & 2.73 & 4.38 & 0.15 & 1.05 & 0.18 \\
12 & -0.14 & 0.35 & 0.25 & 0.51 & 0.32 & 0.60 & 2.61 & 4.78 & 0.18 & 0.93 & 0.20 \\
13 & 0.77 & 0.39 & 0.26 & 0.49 & 0.28 & 1.29 & 2.61 & 4.91 & 0.07 & 0.82 & 0.19 \\
14 & -0.08 & 0.36 & 0.22 & 0.52 & 0.25 & 0.60 & 3.03 & 4.67 & 0.10 & 0.93 & 0.22 \\
15 & 0.18 & 0.29 & 0.25 & 0.53 & 0.31 & 0.72 & 3.08 & 5.06 & 0.11 & 0.87 & 0.17 \\
16 & 0.59 & 0.39 & 0.22 & 0.47 & 0.28 & 0.63 & 2.73 & 4.04 & 0.11 & 1.06 & 0.19 \\
$\mu$ & 0.11 & 0.34 & 0.23 & 0.49 & 0.30 & 0.59 & 2.35 & 4.54 & 0.10 & 0.83 & 0.16 \\
\hline
\end{tabular}

Table 30: Mean posterior estimates from the complex DSTP model in the Servant et al. (2015) data set.

\begin{tabular}{lrrrrrrrrrrr}
\hline Subject & $\mathrm{v}_{t a}$ & $\mathrm{a}$ & ter & $\mathrm{z}$ & $\mathrm{a}_{s s}$ & $\mathrm{v}_{f l}$ & $\mathrm{v}_{s s}$ & $\mathrm{v}_{p 2}$ & $\mathrm{~s}_{\text {ter }}$ & $\mathrm{s}_{v}$ & $\mathrm{~s}_{z}$ \\
\hline 1 & 1.47 & 0.26 & 0.27 & 0.50 & 0.47 & 1.16 & 5.21 & 6.57 & 0.14 & 1.65 & 0.21 \\
2 & 0.64 & 0.80 & 0.19 & 0.49 & 0.79 & 0.69 & 4.81 & 7.28 & 0.18 & 1.72 & 0.21 \\
3 & 0.08 & 0.79 & 0.15 & 0.47 & 0.67 & 0.50 & 3.87 & 5.73 & 0.07 & 1.41 & 0.34 \\
4 & 0.41 & 0.47 & 0.17 & 0.46 & 0.66 & 0.39 & 2.51 & 8.99 & 0.21 & 0.92 & 0.31 \\
5 & 0.01 & 0.58 & 0.03 & 0.48 & 0.80 & 0.26 & 2.92 & 10.84 & 0.21 & 0.80 & 0.09 \\
6 & 0.77 & 0.57 & 0.18 & 0.54 & 0.44 & 1.22 & 4.87 & 6.36 & 0.14 & 1.77 & 0.22 \\
7 & -1.24 & 1.29 & 0.19 & 0.47 & 0.54 & 1.72 & 6.25 & 7.33 & 0.27 & 2.33 & 0.37 \\
8 & -0.14 & 0.54 & 0.18 & 0.47 & 0.33 & 1.36 & 3.57 & 5.06 & 0.17 & 1.40 & 0.24 \\
9 & -0.27 & 1.06 & 0.12 & 0.42 & 0.89 & 1.03 & 5.52 & 7.03 & 0.17 & 1.61 & 0.39 \\
10 & 0.73 & 0.81 & 0.16 & 0.49 & 0.69 & 0.76 & 3.77 & 6.13 & 0.11 & 1.43 & 0.20 \\
11 & 0.59 & 0.05 & 0.31 & 0.54 & 0.11 & 0.21 & 1.47 & 0.30 & 0.14 & 0.03 & 0.09 \\
12 & 0.75 & 0.89 & 0.16 & 0.57 & 0.80 & 0.91 & 4.29 & 7.18 & 0.12 & 1.50 & 0.12 \\
$\mu$ & 0.31 & 0.31 & 0.16 & 0.49 & 0.35 & 0.54 & 0.99 & 4.22 & 0.12 & 0.31 & 0.12 \\
\hline
\end{tabular}


Table 31: Mean posterior estimates from the simple SSP model in the White et al. (2011) data set.

\begin{tabular}{lrrrrrr}
\hline Subject & $\mathrm{a}$ & ter & $\mathrm{z}$ & $\mathrm{p}$ & $\mathrm{SD}_{0}$ & $\mathrm{SD}_{r}$ \\
\hline 1 & 0.06 & 0.34 & 0.50 & 0.38 & 11.31 & 295.35 \\
2 & 0.05 & 0.32 & 0.49 & 0.29 & 12.37 & 311.67 \\
3 & 0.06 & 0.32 & 0.49 & 0.42 & 11.03 & 290.09 \\
4 & 0.06 & 0.28 & 0.50 & 0.37 & 13.25 & 269.77 \\
5 & 0.06 & 0.30 & 0.45 & 0.39 & 12.30 & 328.63 \\
6 & 0.06 & 0.29 & 0.48 & 0.26 & 16.35 & 218.44 \\
7 & 0.06 & 0.32 & 0.47 & 0.40 & 12.35 & 290.61 \\
8 & 0.06 & 0.28 & 0.46 & 0.32 & 17.20 & 330.58 \\
9 & 0.07 & 0.29 & 0.52 & 0.35 & 13.68 & 292.53 \\
10 & 0.05 & 0.29 & 0.49 & 0.33 & 14.73 & 321.11 \\
11 & 0.06 & 0.28 & 0.53 & 0.43 & 14.03 & 281.39 \\
12 & 0.06 & 0.27 & 0.46 & 0.38 & 11.82 & 317.56 \\
13 & 0.06 & 0.28 & 0.52 & 0.33 & 13.32 & 289.65 \\
14 & 0.07 & 0.32 & 0.44 & 0.39 & 15.03 & 315.70 \\
15 & 0.04 & 0.27 & 0.48 & 0.32 & 7.78 & 114.20 \\
16 & 0.05 & 0.27 & 0.52 & 0.40 & 13.87 & 291.53 \\
17 & 0.05 & 0.26 & 0.49 & 0.25 & 14.91 & 263.98 \\
18 & 0.05 & 0.30 & 0.47 & 0.27 & 14.50 & 162.50 \\
19 & 0.06 & 0.28 & 0.51 & 0.33 & 12.24 & 278.43 \\
20 & 0.07 & 0.33 & 0.52 & 0.48 & 11.99 & 305.02 \\
21 & 0.06 & 0.32 & 0.50 & 0.41 & 13.72 & 313.14 \\
22 & 0.06 & 0.30 & 0.51 & 0.41 & 16.85 & 339.12 \\
23 & 0.06 & 0.31 & 0.52 & 0.37 & 11.90 & 296.94 \\
24 & 0.07 & 0.29 & 0.52 & 0.36 & 14.90 & 260.27 \\
25 & 0.05 & 0.31 & 0.52 & 0.39 & 14.86 & 277.17 \\
$\mu$ & 0.06 & 0.30 & 0.49 & 0.36 & 12.44 & 248.91 \\
\hline
\end{tabular}


Table 32: Mean posterior estimates from the simple SSP model in the Ulrich et al. (2015) data set.

\begin{tabular}{lrrrrrr}
\hline Subject & $\mathrm{a}$ & ter & $\mathrm{z}$ & $\mathrm{p}$ & $\mathrm{SD}_{0}$ & $\mathrm{SD}_{r}$ \\
\hline 1 & 0.05 & 0.29 & 0.54 & 0.43 & 4.81 & 192.96 \\
2 & 0.04 & 0.30 & 0.45 & 0.40 & 5.34 & 191.93 \\
3 & 0.04 & 0.27 & 0.52 & 0.32 & 5.13 & 190.51 \\
4 & 0.05 & 0.31 & 0.46 & 0.33 & 4.47 & 193.96 \\
5 & 0.04 & 0.27 & 0.48 & 0.36 & 4.00 & 194.60 \\
6 & 0.04 & 0.25 & 0.43 & 0.37 & 5.13 & 191.17 \\
7 & 0.04 & 0.23 & 0.46 & 0.34 & 6.00 & 186.45 \\
8 & 0.05 & 0.30 & 0.49 & 0.40 & 5.67 & 196.71 \\
9 & 0.05 & 0.23 & 0.49 & 0.41 & 5.55 & 190.32 \\
10 & 0.05 & 0.29 & 0.53 & 0.52 & 5.09 & 190.26 \\
11 & 0.05 & 0.27 & 0.42 & 0.33 & 5.94 & 196.02 \\
12 & 0.05 & 0.30 & 0.51 & 0.36 & 5.47 & 191.27 \\
13 & 0.05 & 0.30 & 0.50 & 0.52 & 5.40 & 195.28 \\
14 & 0.05 & 0.27 & 0.52 & 0.46 & 2.91 & 203.39 \\
15 & 0.05 & 0.29 & 0.53 & 0.52 & 4.65 & 194.00 \\
16 & 0.05 & 0.28 & 0.47 & 0.39 & 3.63 & 196.91 \\
$\mu$ & 0.05 & 0.28 & 0.49 & 0.40 & 4.92 & 189.67 \\
\hline
\end{tabular}

Table 33: Mean posterior estimates from the simple SSP model in the Servant et al. (2015) data set.

\begin{tabular}{lrrrrrr}
\hline Subject & $\mathrm{a}$ & ter & $\mathrm{z}$ & $\mathrm{p}$ & $\mathrm{SD}_{0}$ & $\mathrm{SD}_{r}$ \\
\hline 1 & 0.04 & 0.27 & 0.50 & 0.47 & 5.34 & 232.17 \\
2 & 0.06 & 0.27 & 0.49 & 0.34 & 6.57 & 246.57 \\
3 & 0.06 & 0.26 & 0.47 & 0.31 & 6.52 & 241.10 \\
4 & 0.05 & 0.27 & 0.47 & 0.27 & 7.02 & 246.01 \\
5 & 0.05 & 0.20 & 0.49 & 0.33 & 5.37 & 257.53 \\
6 & 0.05 & 0.22 & 0.53 & 0.46 & 6.76 & 299.17 \\
7 & 0.07 & 0.27 & 0.47 & 0.32 & 6.97 & 254.03 \\
8 & 0.05 & 0.23 & 0.48 & 0.36 & 6.99 & 247.41 \\
9 & 0.07 & 0.24 & 0.42 & 0.37 & 8.18 & 242.09 \\
10 & 0.06 & 0.25 & 0.49 & 0.33 & 6.95 & 248.36 \\
11 & 0.05 & 0.25 & 0.54 & 0.32 & 6.05 & 254.26 \\
12 & 0.07 & 0.26 & 0.56 & 0.36 & 7.82 & 254.00 \\
$\mu$ & 0.06 & 0.25 & 0.49 & 0.35 & 6.59 & 236.89 \\
\hline
\end{tabular}


Table 34: Mean posterior estimates from the complex SSP model in the White et al. (2011) data set.

\begin{tabular}{lrrrrrrrrr}
\hline Subject & $\mathrm{a}$ & ter & $\mathrm{z}$ & $\mathrm{p}$ & $\mathrm{SD}_{0}$ & $\mathrm{SD}_{r}$ & $\mathrm{~s}_{\text {ter }}$ & $\mathrm{s}_{v}$ & $\mathrm{~s}_{z}$ \\
\hline 1 & 0.07 & 0.36 & 0.50 & 0.63 & 7.55 & 176.57 & 0.13 & 0.21 & 0.03 \\
2 & 0.07 & 0.34 & 0.48 & 0.56 & 7.95 & 171.47 & 0.10 & 0.24 & 0.02 \\
3 & 0.06 & 0.34 & 0.48 & 0.56 & 6.78 & 173.93 & 0.14 & 0.13 & 0.05 \\
4 & 0.07 & 0.32 & 0.50 & 0.58 & 8.50 & 175.45 & 0.17 & 0.20 & 0.05 \\
5 & 0.07 & 0.32 & 0.45 & 0.70 & 7.70 & 179.11 & 0.13 & 0.25 & 0.02 \\
6 & 0.06 & 0.34 & 0.48 & 0.40 & 9.23 & 159.70 & 0.22 & 0.18 & 0.06 \\
7 & 0.08 & 0.33 & 0.46 & 0.66 & 8.25 & 173.71 & 0.09 & 0.22 & 0.03 \\
8 & 0.07 & 0.31 & 0.46 & 0.48 & 8.84 & 171.95 & 0.14 & 0.18 & 0.04 \\
9 & 0.08 & 0.32 & 0.52 & 0.49 & 8.30 & 174.55 & 0.15 & 0.14 & 0.05 \\
10 & 0.06 & 0.32 & 0.49 & 0.63 & 8.00 & 169.82 & 0.15 & 0.25 & 0.05 \\
11 & 0.07 & 0.31 & 0.53 & 0.57 & 7.79 & 175.82 & 0.15 & 0.12 & 0.06 \\
12 & 0.06 & 0.32 & 0.45 & 0.59 & 5.61 & 181.13 & 0.20 & 0.17 & 0.07 \\
13 & 0.06 & 0.32 & 0.53 & 0.46 & 6.76 & 172.70 & 0.19 & 0.13 & 0.07 \\
14 & 0.09 & 0.33 & 0.43 & 0.70 & 8.80 & 161.82 & 0.08 & 0.23 & 0.03 \\
15 & 0.05 & 0.29 & 0.46 & 0.49 & 8.70 & 151.97 & 0.15 & 0.23 & 0.03 \\
16 & 0.06 & 0.28 & 0.52 & 0.63 & 8.54 & 171.62 & 0.07 & 0.25 & 0.03 \\
17 & 0.06 & 0.32 & 0.50 & 0.51 & 7.63 & 159.03 & 0.24 & 0.21 & 0.11 \\
18 & 0.06 & 0.34 & 0.47 & 0.46 & 10.27 & 143.60 & 0.18 & 0.20 & 0.07 \\
19 & 0.08 & 0.30 & 0.51 & 0.57 & 8.49 & 178.33 & 0.10 & 0.21 & 0.05 \\
20 & 0.07 & 0.37 & 0.52 & 0.72 & 6.78 & 187.68 & 0.16 & 0.19 & 0.03 \\
21 & 0.07 & 0.35 & 0.51 & 0.70 & 7.56 & 179.07 & 0.16 & 0.23 & 0.03 \\
22 & 0.08 & 0.32 & 0.50 & 0.69 & 9.30 & 170.19 & 0.12 & 0.23 & 0.03 \\
23 & 0.07 & 0.33 & 0.53 & 0.55 & 7.56 & 178.61 & 0.12 & 0.18 & 0.04 \\
24 & 0.08 & 0.33 & 0.52 & 0.57 & 8.74 & 168.05 & 0.17 & 0.18 & 0.08 \\
25 & 0.06 & 0.33 & 0.53 & 0.61 & 8.46 & 163.96 & 0.13 & 0.20 & 0.04 \\
$\mu$ & 0.07 & 0.33 & 0.49 & 0.58 & 8.04 & 168.35 & 0.14 & 0.20 & 0.03 \\
\hline
\end{tabular}


Table 35: Mean posterior estimates from the complex SSP model in the Ulrich et al. (2015) data set.

\begin{tabular}{lrrrrrrrrr}
\hline Subject & $\mathrm{a}$ & ter & $\mathrm{z}$ & $\mathrm{p}$ & $\mathrm{SD}_{0}$ & $\mathrm{SD}_{r}$ & $\mathrm{~s}_{\text {ter }}$ & $\mathrm{s}_{v}$ & $\mathrm{~s}_{z}$ \\
\hline 1 & 0.05 & 0.33 & 0.54 & 0.76 & 2.73 & 135.05 & 0.16 & 0.26 & 0.05 \\
2 & 0.05 & 0.33 & 0.44 & 0.72 & 3.66 & 131.16 & 0.12 & 0.28 & 0.03 \\
3 & 0.04 & 0.31 & 0.52 & 0.46 & 2.60 & 134.84 & 0.16 & 0.10 & 0.07 \\
4 & 0.05 & 0.35 & 0.46 & 0.49 & 2.85 & 134.08 & 0.16 & 0.18 & 0.05 \\
5 & 0.04 & 0.30 & 0.48 & 0.54 & 2.18 & 134.24 & 0.16 & 0.17 & 0.05 \\
6 & 0.04 & 0.27 & 0.45 & 0.49 & 3.35 & 133.98 & 0.16 & 0.14 & 0.05 \\
7 & 0.04 & 0.29 & 0.44 & 0.57 & 3.37 & 132.74 & 0.26 & 0.10 & 0.07 \\
8 & 0.05 & 0.33 & 0.48 & 0.81 & 3.39 & 131.05 & 0.14 & 0.35 & 0.03 \\
9 & 0.04 & 0.30 & 0.51 & 0.72 & 2.48 & 134.86 & 0.23 & 0.16 & 0.07 \\
10 & 0.05 & 0.31 & 0.54 & 0.66 & 3.48 & 130.75 & 0.11 & 0.11 & 0.03 \\
11 & 0.05 & 0.32 & 0.39 & 0.58 & 3.19 & 133.80 & 0.21 & 0.23 & 0.05 \\
12 & 0.05 & 0.35 & 0.53 & 0.57 & 3.10 & 133.45 & 0.18 & 0.20 & 0.05 \\
13 & 0.06 & 0.33 & 0.49 & 0.92 & 3.81 & 131.58 & 0.12 & 0.30 & 0.02 \\
14 & 0.05 & 0.31 & 0.53 & 0.88 & 2.05 & 136.69 & 0.18 & 0.26 & 0.04 \\
15 & 0.05 & 0.32 & 0.53 & 0.76 & 2.74 & 133.08 & 0.14 & 0.19 & 0.04 \\
16 & 0.06 & 0.30 & 0.47 & 0.56 & 2.67 & 133.72 & 0.13 & 0.18 & 0.03 \\
$\mu$ & 0.05 & 0.31 & 0.49 & 0.65 & 2.97 & 132.78 & 0.16 & 0.16 & 0.04 \\
\hline
\end{tabular}

Table 36: Mean posterior estimates from the complex SSP model in the Servant et al. (2015) data set.

\begin{tabular}{lrrrrrrrrr}
\hline Subject & $\mathrm{a}$ & ter & $\mathrm{z}$ & $\mathrm{p}$ & $\mathrm{SD}_{0}$ & $\mathrm{SD}_{r}$ & $\mathrm{~s}_{\text {ter }}$ & $\mathrm{s}_{v}$ & $\mathrm{~s}_{z}$ \\
\hline 1 & 0.03 & 0.30 & 0.50 & 0.62 & 3.57 & 195.73 & 0.16 & 0.13 & 0.01 \\
2 & 0.05 & 0.31 & 0.49 & 0.43 & 3.76 & 195.55 & 0.21 & 0.12 & 0.04 \\
3 & 0.06 & 0.28 & 0.47 & 0.34 & 4.43 & 194.37 & 0.16 & 0.04 & 0.03 \\
4 & 0.05 & 0.30 & 0.47 & 0.36 & 5.54 & 190.99 & 0.22 & 0.15 & 0.02 \\
5 & 0.05 & 0.24 & 0.48 & 0.42 & 3.22 & 197.70 & 0.24 & 0.10 & 0.05 \\
6 & 0.05 & 0.25 & 0.55 & 0.62 & 3.70 & 194.90 & 0.17 & 0.19 & 0.02 \\
7 & 0.07 & 0.31 & 0.46 & 0.50 & 5.27 & 195.70 & 0.21 & 0.18 & 0.05 \\
8 & 0.06 & 0.27 & 0.47 & 0.58 & 5.50 & 196.05 & 0.19 & 0.19 & 0.04 \\
9 & 0.06 & 0.28 & 0.42 & 0.42 & 5.62 & 199.28 & 0.21 & 0.04 & 0.05 \\
10 & 0.06 & 0.28 & 0.50 & 0.40 & 5.25 & 192.98 & 0.19 & 0.10 & 0.02 \\
11 & 0.05 & 0.27 & 0.54 & 0.37 & 4.59 & 193.06 & 0.15 & 0.10 & 0.02 \\
12 & 0.07 & 0.30 & 0.57 & 0.50 & 5.63 & 191.82 & 0.20 & 0.15 & 0.03 \\
$\mu$ & 0.05 & 0.28 & 0.49 & 0.46 & 4.66 & 191.86 & 0.19 & 0.10 & 0.02 \\
\hline
\end{tabular}

